\title{
Ionic Screening in Bulk and under Confinement
}

\author{
Johannes Zeman, ${ }^{1}$ Svyatoslav Kondrat, ${ }^{2,3,4}$ and Christian Holm ${ }^{1}$ \\ ${ }^{1}$ Institute for Computational Physics, University of Stuttgart, D-70569 Stuttgart, Germany \\ ${ }^{2}$ Institute of Physical Chemistry, Polish Academy of Sciences, 01-224 Warsaw, Poland \\ ${ }^{3}$ Max-Planck-Institut für Intelligente Systeme, \\ Heisenbergstraße 3, 70569 Stuttgart, Germany \\ ${ }^{4} I V$. Institut für Theoretische Physik, Universität Stuttgart, \\ Pfaffenwaldring 57, 70569 Stuttgart, Germany
}

(Dated: October 28, 2021)

\begin{abstract}
Recent experiments have shown that the repulsive force between atomically flat, like-charged surfaces confining room-temperature ionic liquids or concentrated electrolytes exhibits an anomalously large decay length. In our previous publication [Zeman et al., Chem. Commun. 56, 15635 (2020)], we showed by means of extremely large-scale molecular dynamics simulations that this so-called underscreening effect might not be a feature of bulk electrolytes. Herein, we corroborate these findings by providing additional results with more detailed analyses and expand our investigations to ionic liquids under confinement. Unlike in bulk systems, where screening lengths are computed from the decay of interionic potentials of mean force (PMFs), we extract such data in confined systems from cumulative charge distributions. At high concentrations, our simulations show increasing screening lengths with increasing electrolyte concentration, consistent with classical liquid state theories. However, our analyses demonstrate that - also for confined systems - there is no anomalously large screening length. As expected, the screening lengths determined for ionic liquids under confinement are in good quantitative agreement with the screening lengths of the same ionic systems in bulk. In addition, we show that some theoretical models used in the literature to relate the measured screening lengths to other observables are inapplicable to highly concentrated electrolytes.
\end{abstract}

\section{INTRODUCTION}

During the past decades, room-temperature ionic liquids (ILs) have gained an increasingly important role in science and technology, with applications ranging from organic synthesis, catalysis, and analytical chemistry to energy storage devices such as electrochemical batteries or supercapacitors ${ }^{1-4}$. Due to the vast number of cationic and anionic species, solvents, or additives available for combination, the physicochemical properties of pure ILs and IL-based solutions are highly tunable $\mathrm{e}^{1,5-8}$. The prediction and targeted adjustment of these properties require a fundamental understanding of the intra- and intermolecular mechanisms governing the internal structure and dynamics of the investigated substances. In that respect, the recently measured anomalously large spatial decay lengths of effective interactions be- 


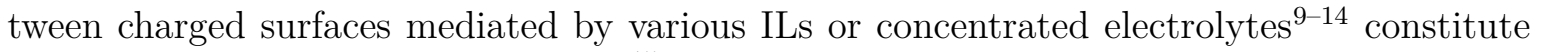
an important but not yet understood ${ }^{15}$ phenomenon. However, understanding the origin of these findings should be especially important for the design of devices where concentrated electrolytes or ILs are strongly confined between highly charged surfaces, as, e.g., in modern supercapacitors.

In the reported surface force balance (SFB) measurements, the electrolyte or IL was confined between two atomically flat, charged surfaces in a cross-cylinder arrangement (see, e.g., ref. 13). The decay length of electrostatic interactions between the surfaces, and thus, across the electrolyte, was obtained from the decay of the distance-dependent force acting between the charged surfaces. Regardless of ionic species and concentrations, the reported force-distance curves exhibited a short-ranged oscillatory decay up to distances of a few nanometers, followed by a long-ranged monotonic exponential decay. In the highconcentration regime, the long-range decay lengths were generally found to increase with electrolyte concentration ${ }^{9-14}$.

Since the asymptotic decay of both charge and density correlations around objects immersed in an electrolyte solution is independent of the size of the objects ${ }^{16,17}$, the experimentally measured decay length was suggested to be a property of bulk electrolytes ${ }^{18}$. Following this argument, ion-ion interactions within a bulk electrolyte solution should, therefore, exhibit the same asymptotic decay length.

The traditional view that the electrostatic screening length decreases with increasing electrolyte concentration is well-known to be valid only at low ion concentrations. In the lowconcentration regime, the spatial decay of ion-ion interactions is usually well-described by approaches such as the Debye-Hückel theory ${ }^{19}$, or, for the electrostatic screening of immersed charged objects, the Poisson-Boltzmann equation ${ }^{20,21}$. In both cases, the asymptotic decay length of electrostatic interactions equals the Debye length $\lambda_{\mathrm{D}}$ defined as

$$
\lambda_{\mathrm{D}}^{2}=\frac{\varepsilon_{0} \varepsilon_{r} k_{\mathrm{B}} T}{\sum_{i} \rho_{i} z_{i}^{2} e^{2}} .
$$

Here, $\varepsilon_{0}$ is the permittivity of free space, $\varepsilon_{r}$ the relative dielectric permittivity of a homogeneous background medium, $k_{\mathrm{B}}$ the Boltzmann constant, and $T$ denotes absolute temperature. In the denominator, $\rho_{i}$ and $z_{i}$ represent the number density and valency of ionic species $i$, and $e$ is the elementary charge. In the limit of infinite dilution, the dielectric permittivity of the background medium equals that of the entire system since it is not altered by the presence of a vanishing amount of free charges.

With rising electrolyte concentration, interactions are increasingly influenced and eventually dominated by excluded-volume and charge correlations, which are difficult to describe by such mean-field approaches. There are many modifications and extensions of the Debye-Hückel and Poisson-Boltzmann theory and other approaches predicting that correlation lengths increase with concentration ${ }^{16,17,22-32}$, therefore resulting in an increasing effective screening length (note that we use the term 'screening length' also for the decay of oscillatory modes of the charge distribution). Especially relevant are integral-equation theories ${ }^{16,22,23,28,29}$ based on descriptions of the radial distribution functions (RDFs) $g_{X Y}(r)$, which describe the probability of finding a particle of species $Y$ at a distance $r$ from a particle of species $X$ and is normalized with respect to the corresponding probability in an ideal gas at the same concentration. Within these frameworks, analyses of the Ornstein-Zernike equation show that for sufficiently high concentrations, the asymptotic decay of ion-ion 
interactions can be expressed as

$$
\lim _{r \rightarrow \infty} w_{X Y}(r) \propto \frac{A}{r} \cos (k r-\phi) \exp \left(-\frac{r}{\lambda_{\mathrm{S}}}\right),
$$

where $w_{X Y}$ denotes the potential of mean force (PMF) between species $X$ and $Y, A$ and $\phi$ are species-dependent amplitudes and phase shifts, $\lambda_{\mathrm{S}}$ is the PMF's asymptotic decay length, and the wave vector $k$ determines the wavelength of its oscillation. In spatially homogeneous systems, the PMF between species $X$ and $Y$ can be obtained from their RDF according to

$$
w_{X Y}(r)=-k_{\mathrm{B}} T \ln \left(g_{X Y}(r)\right) .
$$

In the limit of high ionic concentrations, $\lambda_{S}$ increases with concentration and generally exceeds the Debye length $\lambda_{\mathrm{D}}{ }^{22}$. Thus, in principle, the experimentally measured concentrationdependent increase in screening length is expected. However, what is surprising about the experimental observations is that, in contrast to Eq. (2), the asymptotic decay of surface forces was monotonic and the corresponding decay lengths exceeded theoretical predictions for bulk electrolytes by about an order of magnitude. Anomalously large decay lengths have also been indirectly deduced from fluorescence-based concentration measurements in electrolyte solutions confined between silica surfaces ${ }^{33}$ and observed in an atomic force microscopy (AFM) experiment ${ }^{34}$ (however, there is also an AFM study where large screening lengths were not observed $\left.{ }^{10}\right)$. Regardless of the considered electrolyte, these anomalously large screening lengths $\left(\lambda_{\mathrm{S}}\right)$ followed a cubic scaling with respect to the Debye length $\lambda_{\mathrm{D}}$ of the form $\lambda_{\mathrm{S}} / \lambda_{\mathrm{D}} \propto\left(d / \lambda_{\mathrm{D}}\right)^{3}$, where $d$ is the effective ion diameter (we note, however, that some SFB experiments ${ }^{35,36}$ demonstrated inconsistencies with such a cubic scaling). Lee et al. ${ }^{18,37}$ interpreted this cubic scaling in terms of charge fluctuations due to defects in a nearly crystalline ionic system, as opposite to dilute electrolytes. However, as noted by Adar et al. ${ }^{38}$, this picture is applicable only for electrolyte concentrations above $6 \mathrm{M}$. In more recent work, Ciach and Patsahan ${ }^{39}$ developed a self-consistent field theory and obtained a cubic scaling in the regime of high ion densities. Unlike in the experiments, however, this theoretical cubic scaling appeared in a damped-oscillatory rather than in a monotonic decay of the charge-charge correlation function. Moreover, as estimated by the authors, their calculations are applicable for ion densities $d^{3} \rho \geq 0.75$, which translates into molar concentrations above $23 \mathrm{~mol} / \mathrm{l}$ for aqueous sodium chloride (for an average ion diameter $d=0.3 \mathrm{~nm}$ ) and above $5 \mathrm{~mol} / \mathrm{l}$ for typical ionic liquids (taking $d=0.5 \mathrm{~nm}$ ). However, SFB experiments show that cubic scaling starts even around $1 \mathrm{~mol} / \mathrm{l}$, while the solubility limit of $\mathrm{NaCl}$ is about $5.3 \mathrm{~mol} / 1$ and pure room-temperature ionic liquids typically have similar concentrations. ${ }^{40}$ The possible existence of ionic interactions exhibiting long-ranged monotonic decays has been shown by Kjellander ${ }^{17,30}$ based on dressed ion theory ${ }^{41,42}$. However, we are not aware of applications of this approach to experimental or simulation data of real ionic systems showing cubic scaling or anomalously large screening lengths.

In this work, we aim to gain new physical insights into the long-range behavior of ion interactions in bulk and under confinement using large-scale molecular dynamics (MD) simulations extending our previous letter on bulk properties considerably ${ }^{43}$. We consider various systems, including neat ILs, mixtures of ILs and polar solvents, and concentrated aqueous electrolyte solutions in a broad concentration range and analyze ionic screening lengths and their scaling behavior in details. In addition, we critically review attempts that have been made to connect the measured screening lengths with other experimental observables, seemingly suggesting that underscreening is a bulk property of electrolytes. ${ }^{18,37}$ 


\section{MOLECULAR DYNAMICS SIMULATIONS}

According to both experimental measurements ${ }^{14}$ and theoretical predictions ${ }^{22}$, the enhancement of the asymptotic electrostatic screening length is expected to be most pronounced in pure ILs. Therefore, as a first step, we performed MD Simulations of pure 1-butyl-3-methylimidazolium hexafluorophosphate $\left(\left[\mathrm{C}_{4} \mathrm{C}_{1} \mathrm{Im}\right]^{+}\left[\mathrm{PF}_{6}\right]^{-}\right)$. Our choice was motivated by the fact that there exist well-tested MD force fields for both all-atom and coarsegrained models of this IL. Comparing computational models with different levels of detail will allow us to assess the influence of such molecular details on the resulting asymptotic electrostatic screening length. As a second step, in order to investigate the long-range screening behavior of conventional electrolyte solutions, we performed simulations of aqueous sodium chloride $(\mathrm{NaCl})$ solutions at different concentrations ranging from 1.16 to $5.19 \mathrm{~mol} / \mathrm{l}$.

In ref. 14, Smith et al. also reported SFB measurements of the IL 1-butyl-1-methylpyrrolidinium bis(trifluoromethylsulfonyl)imide $\left(\left[\mathrm{C}_{4} \mathrm{C}_{1} \mathrm{Pyrr}\right]^{+}\left[\mathrm{NTf}_{2}\right]^{-}\right)$in propylene carbonate $(\mathrm{PC})$ solutions at different concentrations. To obtain simulation results which are comparable to the experiment, we conducted simulations of systems comprising solutions of 1-butyl-3-methylimidazolium bis(trifluoromethane)sulfonimide $\left(\left[\mathrm{C}_{4} \mathrm{C}_{1} \mathrm{Im}\right]^{+}\left[\mathrm{NTf}_{2}\right]^{-}\right)$in a racemic $\mathrm{PC}$ mixture (equal amounts of $(\mathrm{R})$ - and $(\mathrm{S})$-propylene carbonate) with IL mole fractions $x$ covering the range from $x=0.05$ to $x=1$ (pure $\left[\mathrm{C}_{4} \mathrm{C}_{1} \mathrm{Im}\right]^{+}\left[\mathrm{NTf}_{2}\right]^{-}$). Due to the fact that the chemical structures and, therefore, the sizes of $\left[\mathrm{C}_{4} \mathrm{C}_{1} \mathrm{Im}\right]^{+}$and $\left[\mathrm{C}_{4} \mathrm{C}_{1} \mathrm{Pyrr}\right]^{+}$ are very similar, we likewise expect a very similar behavior of these species in terms of electrostatic screening.

The compositions of all investigated systems are summarized in Tables I and II, and balland-stick models together with the corresponding Lewis formulas of all involved molecular species (except for $\mathrm{NaCl}$ and water) are depicted in Fig. 1.
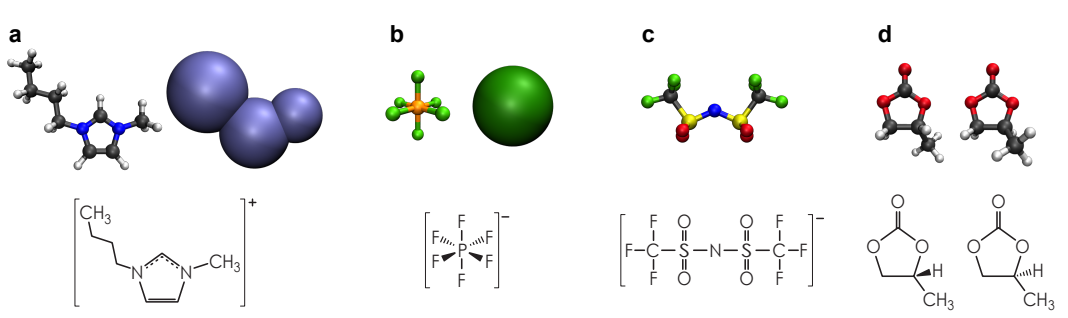

FIG. 1. Ball-and-stick models (top row, produced with VMD ${ }^{44}$ ) and corresponding Lewis formulas (bottom row) of the investigated substances. a: 1-butyl-3-methylimidazolium $\left(\left[\mathrm{C}_{4} \mathrm{C}_{1} \mathrm{Im}\right]^{+}\right)$cation (all-atom (left) and coarse-grained (right)). b: Hexafluorophosphate $\left(\left[\mathrm{PF}_{6}\right]^{-}\right)$anion (all-atom (left) coarse-grained (right)). c: Bis(trifluoromethylsulfonyl)imide $\left(\left[\mathrm{NTf}_{2}\right]^{-}\right)$anion. d: (R)-propylene carbonate (left) and (S)-propylene carbonate (right). 
TABLE I. Bulk systems. Listed values: molar ion pair concentration $c(\mathrm{~mol} / \mathrm{l})$, number of ion pairs $N_{\mathrm{IP}}$, number of solvent molecules $N_{\mathrm{S}}$, average simulation box edge length $\bar{L}(\mathrm{~nm})$, total simulation time $t_{\text {sim }}(\mathrm{ns})$ after equilibration, and equilibration time $t_{\text {eq }}(\mathrm{ns})$. Further details with corresponding error estimates can be found in Section S2.1.1 of the supplementary material.

\begin{tabular}{|c|c|c|c|c|c|}
\hline$c(\mathrm{~mol} / \mathrm{l})$ & $N_{\text {IP }}$ & $N_{\mathrm{S}}$ & $\bar{L}(\mathrm{~nm})$ & $t_{\text {sim }}(\mathrm{ns})$ & $t_{\mathrm{eq}}(\mathrm{ns})$ \\
\hline \multicolumn{6}{|c|}{ pure $\left[\mathrm{C}_{4} \mathrm{C}_{1} \mathrm{Im}\right]^{+}\left[\mathrm{PF}_{6}\right]^{-}$(all-atom) } \\
\hline 4.63 & 108000 & 0 & 33.84 & 600 & 300 \\
\hline \multicolumn{6}{|c|}{ pure $\left[\mathrm{C}_{4} \mathrm{C}_{1} \mathrm{Im}\right]^{+}\left[\mathrm{PF}_{6}\right]^{-}$(coarse-grained) } \\
\hline 4.78 & 358296 & 0 & 49.92 & 700 & 300 \\
\hline \multicolumn{6}{|c|}{$\mathrm{NaCl}$ in water (all-atom) } \\
\hline 4.43 & 216000 & 2458296 & 43.25 & 200 & 50 \\
\hline 1.16 & 3750 & 176577 & 17.53 & 537 & 50 \\
\hline 2.43 & 3750 & 81814 & 13.68 & 5369 & 50 \\
\hline 4.86 & 3750 & 38411 & 10.86 & 5369 & 50 \\
\hline 5.19 & 3750 & 35625 & 10.63 & 5369 & 50 \\
\hline \multicolumn{6}{|c|}{$\left[\mathrm{C}_{4} \mathrm{C}_{1} \mathrm{Im}\right]^{+}\left[\mathrm{NTf}_{2}\right]^{-}$in PC (all-atom) } \\
\hline 0.00 & 0 & 1000 & 5.19 & 1074 & 100 \\
\hline 0.53 & 200 & 3800 & 8.54 & 2147 & 200 \\
\hline 0.97 & 500 & 4500 & 9.51 & 2147 & 200 \\
\hline 1.63 & 500 & 2000 & 7.99 & 3221 & 200 \\
\hline 2.10 & 500 & 1167 & 7.34 & 4295 & 200 \\
\hline 2.47 & 500 & 750 & 6.96 & 4295 & 200 \\
\hline 2.75 & 500 & 500 & 6.71 & 4295 & 200 \\
\hline 2.98 & 500 & 333 & 6.53 & 4295 & 200 \\
\hline 3.17 & 500 & 214 & 6.40 & 4295 & 200 \\
\hline 3.33 & 500 & 125 & 6.30 & 4295 & 200 \\
\hline 3.46 & 500 & 56 & 6.21 & 4295 & 200 \\
\hline 3.58 & 500 & 0 & 6.15 & 4295 & 200 \\
\hline
\end{tabular}

TABLE II. Confined systems. Listed values: number of $\left[\mathrm{C}_{4} \mathrm{C}_{1} \mathrm{Im}\right]^{+}$cations $N_{+}$, number of $\left[\mathrm{NTf}_{2}\right]^{-}$ anions $N_{-}$, simulation box edge length in the $z$-direction $L_{z}(\mathrm{~nm})$, total simulation time $t_{\text {sim }}(\mathrm{ns})$ after equilibration, and equilibration time $t_{\text {eq }}(\mathrm{ns})$. For all systems, the simulation box edge lengths in the $x$ - and $y$-direction are $L_{x}=11.806 \mathrm{~nm}$ and $L_{y}=11.928 \mathrm{~nm}$.

\begin{tabular}{rrrrr}
\hline \hline$N_{+}$ & $N_{-}$ & $L_{z}(\mathrm{~nm})$ & $t_{\text {sim }}(\mathrm{ns})$ & $t_{\text {eq }}(\mathrm{ns})$ \\
\hline 2352 & 2000 & 8.246 & 1000 & 300 \\
2852 & 2500 & 9.902 & 1000 & 300 \\
3852 & 3500 & 13.200 & 1000 & 300 \\
4352 & 4000 & 14.837 & $4 \times 1000$ & 300 \\
\hline \hline
\end{tabular}




\section{A. Simulation details}

Simulations were performed with a customized version of the GROMACS ${ }^{45-51} 2016.3$ simulation package (2019.4 for the confined system) capable of computing and storing cumulative currents (cf. Eq. (6)) at every integration step. All bulk systems were simulated in the $N p T$ ensemble in cubic boxes with periodic boundary conditions $(\mathrm{PBC})$ in all three dimensions, where temperature and pressure were maintained at $T=300 \mathrm{~K}$ and $p=1$ bar, respectively. Electrostatic interactions were computed using the smooth particle mesh Ewald method ${ }^{52}$ (SPME) with a system-dependent short-range cutoff and a relative accuracy of $10^{-5}$. The confined systems were simulated in orthorhombic boxes with PBC in the $x$ - and $y$-directions only, and the ensemble was changed to $N V T$ after equilibration. Due to the 2d-periodicity of these systems, the SPME method was also used for Lennard-Jones (LJ) interactions ${ }^{53}$, and Ewald summation corrections were applied according to Yeh and Berkowitz ${ }^{54}$.

For simulations of ILs, we employed the 0.8*OPLS-2009IL all-atom force field of Doherty et al. ${ }^{55}$, and the ILM2 force field of Roy and Maroncelli ${ }^{56}$ for the coarse-grained description of $\left[\mathrm{C}_{4} \mathrm{C}_{1} \mathrm{Im}\right]^{+}\left[\mathrm{PF}_{6}\right]^{-}$.

These models use ionic charges that are uniformly scaled by a factor of 0.8 and 0.78 , respectively, to implicitly account for electronic polarization effects and partial charge transfer that lead to effectively reduced Coulomb interactions in IL systems. ${ }^{57-59}$ The simplest theoretical explanation for charge scaling can be found in the non-polarizable MDEC (Molecular Dynamics in Electronic Continuum) model of Leontyev and Stuchebrukhov ${ }^{60}$. They use a uniform electronic screening of partial atomic charges to account for the effects of electronic polarization within a mean-field approach. Approaches beyond mean-field theory that derive partial charges from $a b$ initio data arrive at very similar rescaled overall ionic charges, albeit with a different distribution of partial charges. ${ }^{61-66}$

For the description of aqueous $\mathrm{NaCl}$, we used the KBFF ion parameters of Weerasinghe and Smith $^{67}$ in conjunction with the simple point charge $(\mathrm{SPC} / \mathrm{E})$ water model ${ }^{68}$. Since in aqueous $\mathrm{NaCl}$ systems, the ion polarizabilities and their concentrations are much smaller than for molecular ILs, the respective ion models use full charges of $\pm 1 e$. In simulations of $\left[\mathrm{C}_{4} \mathrm{C}_{1} \mathrm{Im}\right]^{+}\left[\mathrm{NTf}_{2}\right]^{-}$in $\mathrm{PC}$ solution, the solvent was described according to parameters provided by Takeuchi et al. ${ }^{69}$. For the carbon atoms forming the graphene walls of the confined systems, we employed the same LJ parameters as for those in the imidazolium ring of $\left[\mathrm{C}_{4} \mathrm{C}_{1} \mathrm{Im}\right]^{+}$. The parameter sets of all simulations are listed in Section $\mathrm{S} 4$ of the supplementary material.

\section{B. Analysis methods}

\section{Potential of mean force}

The evaluation of the PMF $w_{X Y}(r)$ between ionic species $X$ and $Y$ using Eq. (3) requires the knowledge of the corresponding RDF $g_{X Y}(r)$. In homogeneous systems, the latter is formally defined as

$$
g_{X Y}(r)=\frac{\left\langle\rho_{X Y}(r)\right\rangle}{\left\langle\rho_{Y}\right\rangle},
$$

where $\left\langle\rho_{X Y}(r)\right\rangle$ denotes the expected density of particles of species $Y$ residing on shells of radius $r$ centered at any particle of species $X$, and $\left\langle\rho_{Y}\right\rangle$ is the expected density of particles of 
species $Y$ averaged over the entire system, i.e., their bulk density. Since the computation of RDFs with analysis tools included in the GROMACS package would have taken several years for the large-scale IL systems considered, we developed an optimized analysis tool based on the MDAnalysis ${ }^{70,71}$ and MPI for Python ${ }^{72-74}$ packages allowing the evaluation of RDFs on hundreds of CPU cores in parallel. Further details regarding the numerical evaluation of Eq. (4) and error estimation are given in Section S1.1 of the supplementary material.

\section{Dielectric permittivity}

According to Eq. (1), the calculation of the Debye screening length $\lambda_{\mathrm{D}}$ requires the knowledge of the static relative dielectric permittivity $\varepsilon_{r}$ of the background medium. Since in MD simulations with both ions and solvent modeled explicitly, there is no effective dielectric background medium, we used the static relative dielectric permittivity of the simulated substances. In equilibrium MD simulations, $\varepsilon_{r}$ can be obtained from the expected value of the square of the system's itinerant dipole moment $\boldsymbol{M}$ according to ${ }^{75}$

$$
\varepsilon_{r}=1+\frac{\left\langle\boldsymbol{M}^{2}\right\rangle}{3 V k_{\mathrm{B}} T \varepsilon_{0}}
$$

under the conditions that the system has PBC in all three dimensions and electrostatic interactions are computed with tinfoil boundary conditions at infinity. If a system consists entirely of charge-neutral molecules, its itinerant dipole moment $\boldsymbol{M}$ at any given time $t$ equals the sum of the molecular dipole moments $\boldsymbol{\mu}$ so that $\boldsymbol{M}(t)=\sum_{m} \boldsymbol{\mu}_{m}(t)$, and the time average of $\boldsymbol{M}^{2}(t)$ serves as an estimator for $\left\langle\boldsymbol{M}^{2}\right\rangle$. However, in systems with PBC comprising free ionic charges, $\boldsymbol{M}(t)$ has additional intermolecular contributions. The problem which arises is not only that these contributions are discontinuous in time when ions move across periodic boundaries; in fact, $\boldsymbol{M}(t)$ is ill-defined in such systems because its value depends on the choice of the origin of the spatial coordinate system ${ }^{76}$. Nevertheless, its temporal derivative, the itinerant current $\boldsymbol{J}(t)=\frac{d}{d t} \boldsymbol{M}(t)$, does not suffer from any of these problems ${ }^{76}$. It can be computed according to

$$
\boldsymbol{J}(t)=\sum_{i} q_{i} \boldsymbol{v}_{i}(t)
$$

where the index $i$ runs over all atoms with (partial) charges $q_{i}$ and velocities $\boldsymbol{v}_{i}(t)$. Provided that the system is at thermal equilibrium at all times $t$, the time-dependent current $\boldsymbol{J}(t)$ can then be used to obtain the expected squared itinerant dipole moment as (see Section S1.2.1 of the supplementary material)

$$
\left\langle\boldsymbol{M}^{2}\right\rangle=-\lim _{t \rightarrow \infty} \int_{0}^{t} \tau\langle\boldsymbol{J}(0) \boldsymbol{J}(\tau)\rangle \mathrm{d} \tau,
$$

where $\langle\boldsymbol{J}(0) \boldsymbol{J}(t)\rangle$ is the unnormalized autocorrelation function of $\boldsymbol{J}(t)$. In practice, the direct numerical evaluation of Eq. (7) is problematic due to the fact that the statistical noise in the tail of the current autocorrelation function is amplified by the factor $\tau$ in the integral. A more feasible approach is the so-called Einstein-Helfand method ${ }^{77}$, where one performs a linear regression of the itinerant dipole moment's mean square displacement $\left\langle(\Delta \boldsymbol{M})^{2}(t)\right\rangle$ in 
the limit of large $t$, and the right hand side of Eq. (7) is obtained from the fit value at $t=0$. The static relative permittivity is then obtained according to Eq. (5), and, subsequently, the Debye length can be calculated from Eq. (1). A detailed description of this approach is given in Section S1.2 of the supplementary material.

At this point, it is important to mention again that the IL models used in this work employ rescaled charges to implicitly account for the effects of electronic polarizability and partial charge transfer ${ }^{57-59,61-66}$. However, this leads to an underestimation of the current $\boldsymbol{J}(t)$, and, likewise, the dielectric permittivity. Thus, to obtain correct estimates of the permittivity, the ionic charge scaling has to be undone when evaluating Eqs. (6) and (7).

\section{Analysis of confined systems}

In systems where the investigated substance is confined between charged surfaces, the electrostatic screening of the wall charges cannot be inferred from interionic PMFs as such systems are neither homogeneous nor isotropic. Instead, the electrostatic screening of the surface charge can be extracted from the decay of the electric field $E^{\perp}(z)$ in the $z$-direction, i.e., normal to the confining surfaces. To this aim, we analyzed the system's volume charge density profile $\rho_{q}^{\perp}(z)$, which is given by

$$
\rho_{q}^{\perp}(z)=\left\langle\sum_{i} q_{i} \delta\left(z-z_{i}\right)\right\rangle,
$$

where $\delta(\cdot)$ is the Dirac delta function, the operator $\langle\cdot\rangle$ denotes the canonical average, and the index $i$ runs over all atoms in the system with charge $q_{i}$ and position $z_{i}$, respectively. For the numerical evaluation of Eq. (8), the system is discretized in the $z$-direction into $n$ slabs of width $l_{z}=L_{z} / n$, where $L_{z}$ is the length of the simulation box in the $z$-direction. The volume charge density profile is then calculated according to

$$
\rho_{q}^{\perp}(z)=\left\langle\frac{1}{L_{x} L_{y} l_{z}} \sum_{\substack{i \\ 2\left|z-z_{i}\right|<l_{z}}} q_{i}\right\rangle,
$$

and the perpendicular component $E^{\perp}(z)$ of the microscopic electric field is obtained from

$$
E^{\perp}(z)=\frac{1}{\varepsilon_{0}} \int_{0}^{z} \rho_{q}^{\perp}\left(z^{\prime}\right) \mathrm{d} z^{\prime} .
$$

To assess the influence of confinement on the molecules' dipolar orientation, we computed the normal component $\mu_{\text {rot }, j}^{\perp}$ of the rotational dipole moment of molecule $j$ according to

$$
\mu_{\mathrm{rot}, j}^{\perp}=\sum_{\alpha} q_{\alpha, j}\left(z_{\alpha, j}-z_{\mathrm{com}, j}\right),
$$

where $z_{\mathrm{com}, j}$ represents the $z$-position of the molecular center of mass and the index $\alpha$ runs over all atoms of molecule $j$. This allowed us to compute the system's rotational dipole moment density $m_{\text {rot }}^{\perp}(z)$ according to

$$
m_{\mathrm{rot}}^{\perp}(z)=\left\langle\frac{1}{L_{x} L_{y} l_{z}} \sum_{\substack{j \\ 2\left|z-z_{\mathrm{com}, j}\right|<l_{z}}} \mu_{\mathrm{rot}, j}^{\perp}\right\rangle,
$$

where the index $j$ runs over all molecules in the system. 


\section{RESULTS AND DISCUSSION}

\section{A. Bulk long-range interionic potentials of mean force}

In SFB measurements, the distance-dependent force mediated by electrolytes confined between two mica-coated, like-charged surfaces has been found to follow a relatively fast oscillatory decay for surface separations up to a few nanometers before entering a longranged monotonic decay. It has been argued that the observed long-range decay must be a feature of bulk ionic fluids, therefore describing the general asymptotic decay of electrostatic interactions within concentrated electrolytes and ILs. Within these substances, one would therefore expect to observe the same asymptotic decay in effective ion-ion interactions, and, consequently, in the corresponding interionic PMFs. The latter argument has already been brought forward by Kjellander ${ }^{30}$ in a well-founded manner.

For pure ILs, the experimentally observed transition from oscillatory to monotonic decay typically occurs at surface separations between 4 and $7 \mathrm{~nm}^{12,14,15}$. Thus, in order to see such an effect in bulk MD simulations, the systems under consideration must be large enough to allow the evaluation of interionic PMFs going well beyond that range. In other words, analyzing only the short-range parts of ionic interactions before the crossover region is clearly insufficient, and simulation boxes with edge lengths of at least $20 \mathrm{~nm}$ are required to properly resolve the range of interest. Moreover, due to their high viscosity, ILs exhibit long structural relaxation times, necessitating simulation times of several hundred nanoseconds. ${ }^{78}$ Nevertheless, even if these spatiotemporal requirements are fulfilled, the obtained PMFs are rather meaningless without proper estimates of their statistical accuracy. Therefore, for every data point in the computed PMFs, we performed statistical error analyses taking temporal correlations into account (see Section S1.1.3 of the supplementary material for details).

\section{Neat ionic liquids}

The first system we discuss comprises 108000 ion pairs of pure $\left[\mathrm{C}_{4} \mathrm{C}_{1} \mathrm{Im}\right]^{+}\left[\mathrm{PF}_{6}\right]^{-}$(all-atom model with 3456000 atoms in total), with the positions of all atoms recorded for a posteriori analysis every 2 ps during a total simulation time of $700 \mathrm{~ns}$. Figure 2a displays the absolute values of the PMFs $\left|w_{--}\right|(r)$ (anion-anion), $\left|w_{+-}\right|(r)$ (cation-anion), and $\left|w_{++}\right|(r)$ (cationcation) between the respective molecular centers of mass in units of $k_{\mathrm{B}} T$ together with their one-sigma statistical error margins (light-colored areas) for interionic distances up to $16.5 \mathrm{~nm}$. The ordinate of the main graph is logarithmically scaled to facilitate the detection of any long-ranged but possibly low-amplitude monotonic decay, whereas the inset shows the PMF's short-range part in linear scale.

For the investigation of electrostatic effects, distances below the van der Waals cut-off of $1.3 \mathrm{~nm}$ should be excluded from the analysis due to the relatively strong influence of the LJ potential in this region. At larger distances up to $r \approx 8.5 \mathrm{~nm}$, all PMFs are well-described by an exponentially damped, oscillatory decay (2). The envelope of this decay is indicated by a dashed black line, which has a decay length of $\lambda_{\mathrm{S}}=1.05 \mathrm{~nm}$. For distances greater than $8.5 \mathrm{~nm}$, the PMFs become very noisy with an amplitude of about $3 \cdot 10^{-5} k_{\mathrm{B}} T$, which is in the order of the statistical uncertainty. One could argue that due to the comparatively high statistical uncertainty in the tails, monotonic decays in this region might be hidden in the noise. However, experiments with similar ILs ${ }^{14}$ suggest an onset of the monotonic decay 

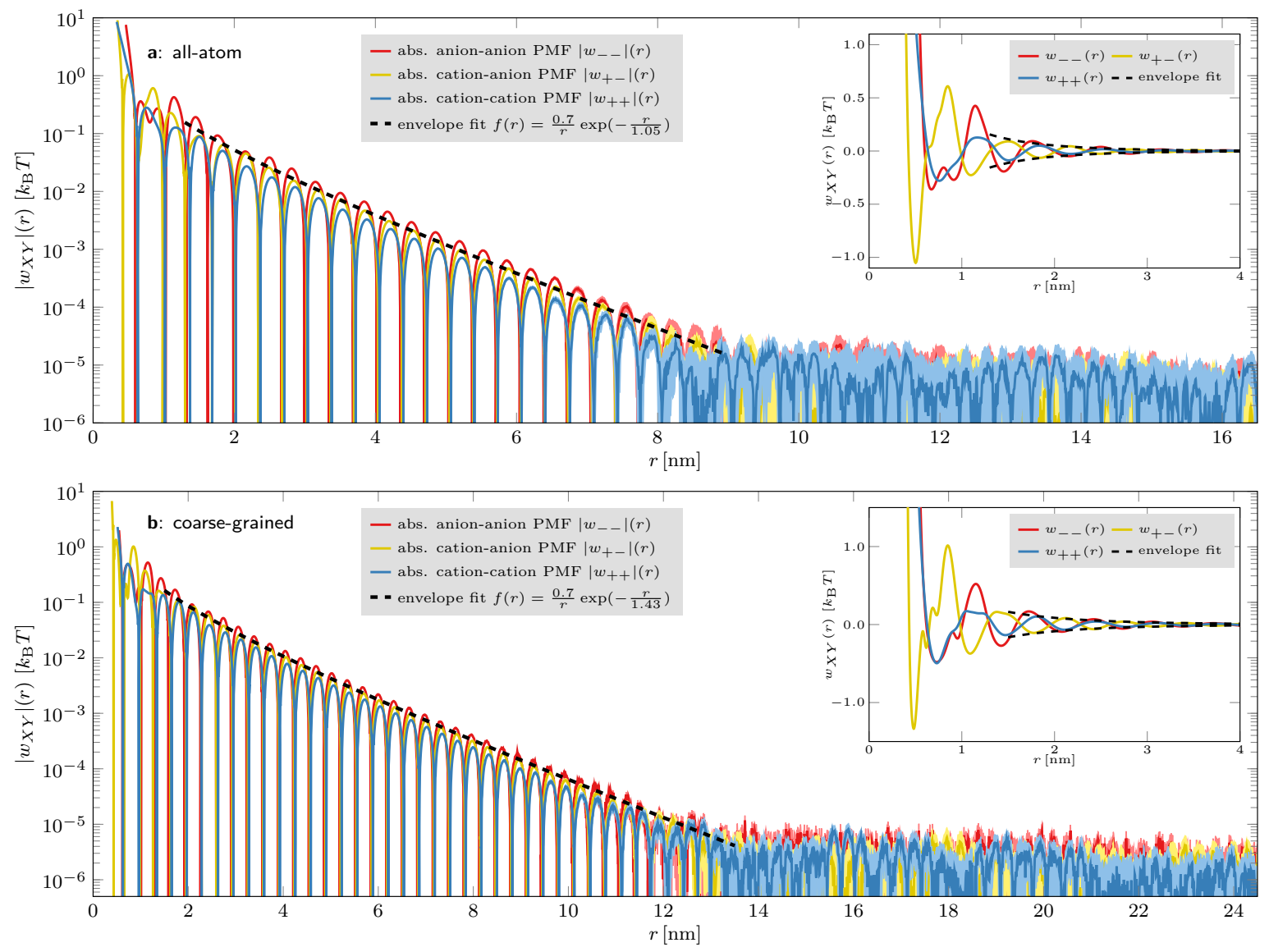

FIG. 2. Absolute value of the anion-anion $\left(\left|w_{--}\right|(r)\right.$, red), cation-anion $\left(\left|w_{+-}\right|(r)\right.$, yellow), and cation-cation $\left(\left|w_{++}\right|(r)\right.$, blue) potentials of mean force in neat $\left[\mathrm{C}_{4} \mathrm{C}_{1} \mathrm{Im}\right]^{+}\left[\mathrm{PF}_{6}\right]^{-}$. a: All-atom model. All PMFs follow an oscillatory decay up to a distance of about $8.5 \mathrm{~nm}$. In this region, the envelope of the decay (dashed, black line) is very well described by a function of the form $f(r)=$ $\frac{a}{r} \exp \left(-\frac{r}{\lambda_{\mathrm{S}}}\right)$ with an amplitude of $a \approx 0.7 k_{\mathrm{B}} T$ and a decay length of $\lambda_{\mathrm{S}}=1.05 \mathrm{~nm}$. For distances larger than $8.5 \mathrm{~nm}$, the potentials enter a region of almost constant amplitude, which is of the same order as the corresponding uncertainties (light-colored areas). b: Coarse-grained model. All PMFs show a qualitatively very similar behavior as for the all-atom model. The envelope of the decay exhibits a longer decay length of $\lambda_{\mathrm{S}}=1.43 \mathrm{~nm}$, which can be attributed to the coarse-grained nature of the molecular model. As for the all-atom model, all PMFs eventually enter a region $(r>13 \mathrm{~nm})$ of almost constant amplitude with rather high uncertainty. Insets: The same PMFs as in the main graphs with linear $y$-axis scaling.

already before that region, and such an onset is clearly not present in our data.

The long-ranged monotonic decay of electrostatic interactions observed in SFB measurements was found to be a universal feature of concentrated electrolytes and ILs and therefore ought to be independent of the chemical details of the involved ionic species. Thus, we extended our analysis to an even larger system with a box edge length of almost $50 \mathrm{~nm}$ comprising 358296 ion pairs of the same IL, but this time modeled by a coarse-grained representation involving only four interaction sites per ion pair (cf. Fig. 1a,b). The corre- 
sponding cation-anion PMFs are displayed in Fig. 2b for interionic distances up to $24.5 \mathrm{~nm}$. All PMFs of the coarse-grained representation show the same qualitative behavior as the all atom model. As expected for coarse-grained models, the corresponding decay length of $\lambda_{\mathrm{S}}=1.43 \mathrm{~nm}$ is comparable to but slightly larger than that of the all-atom model. Due to the larger number of ions in the system, the resulting increased statistical accuracy allows to resolve the oscillatory decay at interionic distances up to $13 \mathrm{~nm}$. For larger distances, the PMFs again enter a region of almost constant amplitude (as low as $5 \cdot 10^{-6} k_{\mathrm{B}} T$ ), which is about the same magnitude as the corresponding statistical one-sigma uncertainties.

\section{Concentrated electrolyte solutions}

The system that we have investigated is a 4.43 molar aqueous $\mathrm{NaCl}$ solution comprising 216000 ion pairs and 2458296 water molecules (7806888 atoms in total) in a cubic simulation box with an edge length of $43.25 \mathrm{~nm}$. The corresponding anion-anion, cation-anion, and cation-cation PMFs obtained from a $200 \mathrm{~ns}$ simulation run are displayed in Fig. 3.

In contrast to the pure IL, a single oscillatory decay is not a satisfactory description of the short-range part of any of the interionic PMFs in aqueous $\mathrm{NaCl}$ but rather a superposition of several such decays with different amplitudes, wavelengths, phase shifts, and decay lengths. Nevertheless, up to a distance of about $2.2 \mathrm{~nm}$, their envelope can be approximated by that

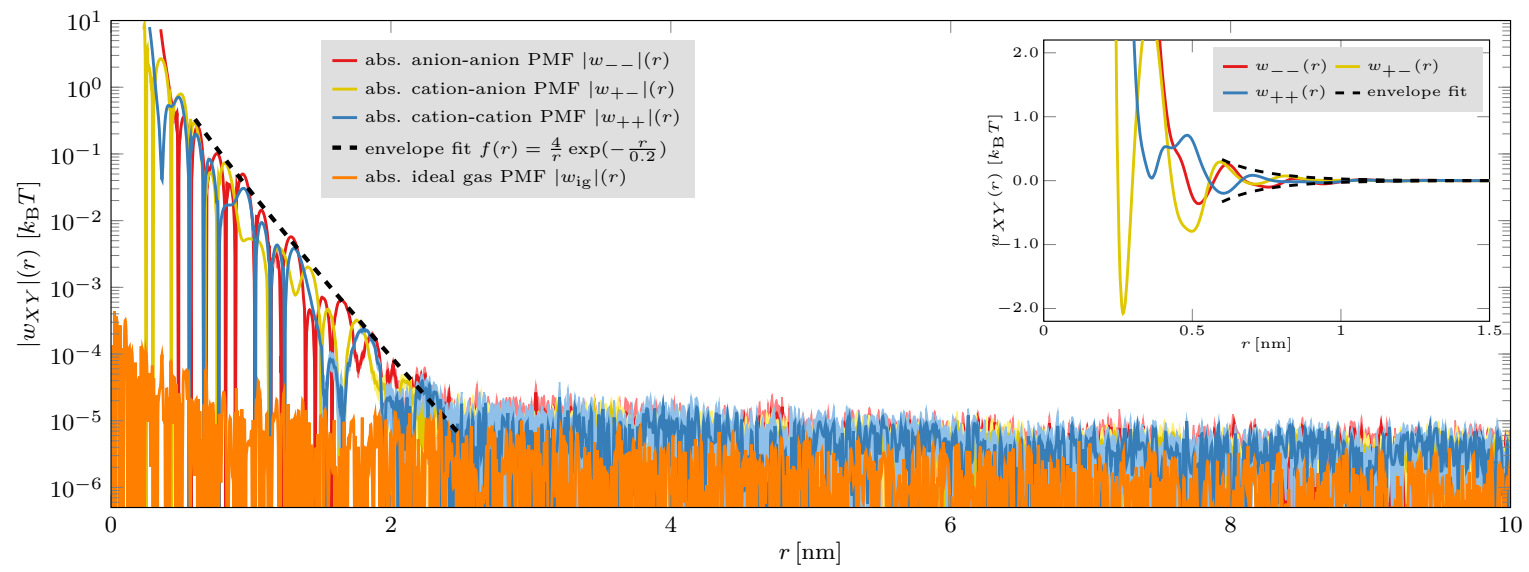

FIG. 3. Absolute value of the anion-anion $\left(\left|w_{--}\right|(r)\right.$, red), cation-anion $\left(\left|w_{+-}\right|(r)\right.$, yellow), and cation-cation $\left(\left|w_{++}\right|(r)\right.$, blue) potentials of mean force in a $4.43 \mathrm{~mol} / \mathrm{l}$ aqueous $\mathrm{NaCl}$ solution. Up to a distance of about $2.2 \mathrm{~nm}$, all PMFs exhibit an oscillatory decay which, in contrast to the case of neat $\left[\mathrm{C}_{4} \mathrm{C}_{1} \mathrm{Im}\right]^{+}\left[\mathrm{PF}_{6}\right]^{-}$, appears to be a superposition of several oscillations with different parameters. Nevertheless, the envelope of the decays (dashed, black line) can be approximated by a function of the form $f(r)=\frac{a}{r} \exp \left(-\frac{r}{\lambda_{\mathrm{S}}}\right)$ with an amplitude of $a=4.0 k_{\mathrm{B}} T$ and a decay length of $\lambda_{\mathrm{S}}=0.2 \mathrm{~nm}$. For distances larger than $2.2 \mathrm{~nm}$, the potentials lie in the order of the uncertainty levels of about $10^{-5} k_{\mathrm{B}} T$. Their further decay is not a feature of the system but simply due to the statistical error, which decreases with increasing distance. This is evident from the noisy orange line, which depicts the PMF $\left|w_{i} g\right|(r)$ of an ideal gas comprising an identical number of particles in the same volume, and exhibits the very same decay. Inset: The same PMFs with linear $y$-axis scaling. The envelope fit is again depicted by dashed black lines. 
of a single oscillatory decay as has been done before for the pure IL. The corresponding fit (dashed black line in Fig. 3) has an amplitude of $4 k_{\mathrm{B}} T$ and a decay length of $\lambda_{\mathrm{S}}=0.2 \mathrm{~nm}$. For distances exceeding $2.2 \mathrm{~nm}$, the PMFs become very noisy, and no distinct oscillations are discernible. In this region, the PMFs' envelopes appears to follow a very long-ranged decay. However, this decay is entirely due to statistical noise, which decreases with distance. In fact, the noise in the PMF of an ideal gas comprising an identical number of particles in the same volume (shown in orange in Fig. 3) exhibits the very same decay. Thus, the observed long-range decay of the noise amplitude is merely a statistical artifact and must not be interpreted as a feature of the system. Instead, we have to conclude that also in MD simulations of concentrated aqueous $\mathrm{NaCl}$ solutions, there is no long-ranged monotonic decay of interionic interactions detectable within an accuracy of $\approx 10^{-5} k_{\mathrm{B}} T$.

\section{B. Scaling analysis of asymptotic screening lengths in concentrated electrolyte solutions}

In order to investigate how the asymptotic decay length $\lambda_{\mathrm{S}}$ scales with ion concentration, we analyzed the PMFs $w_{+-}(r), w_{++}(r)$, and $w_{--}(r)$ obtained from all-atom MD simulations of $\left[\mathrm{C}_{4} \mathrm{C}_{1} \mathrm{Im}\right]^{+}\left[\mathrm{NTf}_{2}\right]^{-}$in propylene carbonate $(\mathrm{PC})$ over the entire concentration range covering IL mole fractions $x$ from $x=0$ to $x=1$. Since the previously analyzed interionic PMFs of large-scale simulations did not show any additional long-range effects different from those at intermediate ion separations, we chose to simulate smaller systems in favor of covering a larger number of different ion concentrations. Except for the two lowest concentrations, all systems comprised 500 ion pairs and a suitably adjusted number of PC molecules. For each system composition, up to four independent simulation runs were performed with runtimes covering more than $1 \mu$ s per run. As before, interionic PMFs were computed according to Eqs. (3) and (4). To estimate the concentration-dependent effective screening length $\lambda_{\mathrm{S}}$ in these systems, we fitted the PMFs with a superposition of $n$ oscillatory, exponentially damped functions

$$
f(r)=\sum_{i=1}^{n} \frac{A_{i}}{r} \cos \left(\omega_{i} r-\phi_{i}\right) \exp \left(-\frac{r}{\lambda_{i}}\right) .
$$

We note that the decay lengths $\lambda_{i}$ and wavelengths $\omega_{i}$ must be the same for $w_{+-}(r), w_{++}(r)$, and $w_{--}(r)$ at each ion concentration. Hence, one should fit all PMFs simultaneously. Nevertheless, we performed fits for each PMF separately because the large number of unknowns would otherwise drive the fitting algorithm unstable. We have checked a posteriori that the fitted values of $\lambda_{i}$ and $\omega_{i}$ are similar for corresponding PMFs.

We found that setting $n=2$ in Eq. (13) was sufficient to obtain an excellent match with the interionic PMFs in the fit range $1.2 \leq r \leq 3.0 \mathrm{~nm}$. Fitting the data to Eq. (13) with $n=3$ terms resulted in extremely unstable results under slight variation of initial fit parameters, while setting $n=1$ lead to unsatisfactory fits with comparatively large residuals.

For each system, we determined the static dielectric permittivities $\varepsilon_{r}$ and the corresponding Debye screening lengths $\lambda_{\mathrm{D}}$. The permittivities of the IL mixtures compare well with experimental measurements for pure $\mathrm{PC}\left(\varepsilon_{r}=59.3\right.$ (simulation) vs. $\varepsilon_{r}=64.97$ (experiment, see ref. 79)) and pure $\left[\mathrm{C}_{4} \mathrm{C}_{1} \mathrm{Im}\right]^{+}\left[\mathrm{NTf}_{2}\right]^{-}\left(\varepsilon_{r}=9.1\right.$ (simulation) vs. $\varepsilon_{r}=11.52$ (experiment, see ref. 80)). The complete set of permittivities is given in Section S2.1.2 of the supplementary material. 


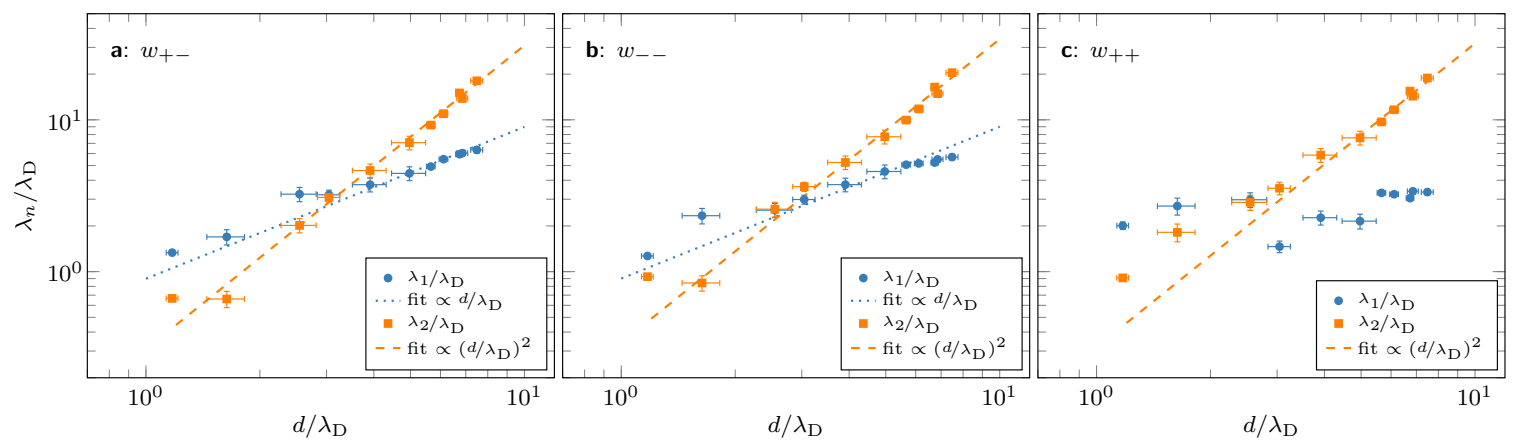

FIG. 4. Scaling of the concentration-dependent screening lengths of $\left[\mathrm{C}_{4} \mathrm{C}_{1} \mathrm{Im}\right]^{+}\left[\mathrm{NTf}_{2}\right]^{-}$in propylene carbonate as determined by fits of Eq. (13) to the concentration-dependent cation-anion $\left(w_{+-}(r)\right)$, anion-anion $\left(w_{--}(r)\right)$, and cation-cation $\left(w_{++}(r)\right)$ PMFs. The abscissa displays the average ion diameter $d$ divided by the concentration-dependent Debye length $\lambda_{\mathrm{D}}$, whereas the determined screening lengths $\lambda_{i}$ divided by $\lambda_{\mathrm{D}}$ are plotted along the ordinate. For the cation-anion (panel a) and anion-anion (panel b) PMFs, we see two decay lengths (each corresponding to a distinct wavelength $2 \pi / \omega_{i}$ ) which both exhibit a power law dependence in the plot. For the cationcation data (panel c), such a dependence can only be established for one of the decay length ratios. In all cases, the asymptotic decay length ratio for high concentrations $\lambda_{2} / \lambda_{\mathrm{D}}$ is clearly proportional to $\left(d / \lambda_{\mathrm{D}}\right)^{2}$.

The knowledge of both the concentration-dependent asymptotic screening lengths $\lambda_{\mathrm{S}}$ and the corresponding Debye lengths $\lambda_{\mathrm{D}}$ allowed us to conduct a scaling analysis similar to that by Lee et al. ${ }^{37}$. Accordingly, Fig. 4 shows the ratio of the screening length vs. the Debye length $\lambda_{\mathrm{S}} / \lambda_{\mathrm{D}}$ as a function of the inverse Debye length scaled with the average ion diameter $d / \lambda_{\mathrm{D}}$. Following ref. 37, the average ion diameter $d$ of the IL was determined from the number concentration of ion pairs in the pure IL as $d=\frac{1}{2}\left(V / N_{\mathrm{IP}}\right)^{\frac{1}{3}} \approx 0.39 \mathrm{~nm}$. The resulting asymptotic linear and quadratic scaling at high concentrations

$$
\frac{\lambda_{n}}{\lambda_{\mathrm{D}}} \propto\left(\frac{d}{\lambda_{\mathrm{D}}}\right)^{n}
$$

is clearly visible from the blue and orange dashed lines in Fig. 4. While the linear scaling of $\lambda_{1} / \lambda_{\mathrm{D}}$ is only present for the cation-anion and anion-anion PMFs, the quadratic scaling at high IL concentrations is retained also in the cation-cation PMFs, as we reported earlier ${ }^{43}$. Such a quadratic scaling has also been obtained by Adar et al. ${ }^{38}$, who used an elegant theory based on the modification of the electrostatic kernel accounting for ion size effects, and more recently by Krucker-Velasquez and Swan ${ }^{81}$ in simulations of primitive model electrolytes. However, this quadratic scaling stands in contrast to the cubic scaling $\lambda_{\mathrm{S}} / \lambda_{\mathrm{D}} \propto\left(d / \lambda_{\mathrm{D}}\right)^{3}$ obtained from experimental SFB measurements ${ }^{37}$. Moreover, the magnitudes of the effective screening lengths according to SFB measurements exceed our values by up to a factor of ten. A comparison of the asymptotic screening lengths we obtained from bulk simulations with experimental data is shown in Table III. 
TABLE III. Comparison of screening lengths in the investigated bulk systems with corresponding values from SFB experiments involving the same or comparable substances. For each concentration, $\lambda_{\mathrm{S}}^{\text {sim }}$ represents the maximum of the three screening lengths obtained from the decays of the PMFs $w_{+-}, w_{--}$, and $w_{++}$. Listed values: molar ion pair concentration $c(\mathrm{~mol} / \mathrm{l})$, screening length from simulations $\lambda_{\mathrm{S}}^{\operatorname{sim}}(\mathrm{nm})$, and screening length from SFB measurements $\lambda_{\mathrm{S}}^{\mathrm{SFB}}(\mathrm{nm})$.

\begin{tabular}{lccr}
\hline \hline System & $c(\mathrm{~mol} / \mathrm{l})$ & $\lambda_{\mathrm{S}}^{\text {sim }}(\mathrm{nm})$ & $\lambda_{\mathrm{S}}^{\mathrm{SFB}}(\mathrm{nm})$ \\
\hline$\left[\mathrm{C}_{4} \mathrm{C}_{1} \mathrm{Im}\right]^{+}\left[\mathrm{PF}_{6}\right]^{-}$ & $4.63^{*}$ & 1.05 & - \\
\hline$\left[\mathrm{C}_{4} \mathrm{C}_{1} \mathrm{Im}\right]^{+}\left[\mathrm{PF}_{6}\right]^{-}$(coarse-grained) & $4.78^{*}$ & 1.43 & - \\
\hline & 1.16 & 0.25 & $0.52^{\mathrm{a}}$ \\
$\mathrm{NaCl}$ in water & 2.43 & 0.20 & $1.37^{\mathrm{a}}$ \\
& 4.86 & 0.32 & $2.99^{\mathrm{a}}$ \\
& 5.19 & 0.33 & $-21^{\mathrm{a}}$ \\
& 0.53 & 0.67 & $1.33^{\mathrm{b}}$ \\
& 0.97 & 0.65 & $4.12^{\mathrm{b}}$ \\
& 1.63 & 0.50 & $6.12^{\mathrm{b}}$ \\
{$\left[\mathrm{C}_{4} \mathrm{C}_{1} \mathrm{Im}\right]^{+}\left[\mathrm{NTf}_{2}\right]^{-}$in PC } & 2.10 & 0.47 & $7.68^{\mathrm{b}}$ \\
& 2.47 & 0.58 & \\
& 2.75 & 0.61 & $8.83^{\mathrm{c}}$ \\
& 2.98 & 0.69 & \\
\hline \hline
\end{tabular}

* Pure ionic liquid (without solvent).

a Data for $\mathrm{NaCl}$ in aqueous solution from ref. 14, linearly interpolated in the range $c \in[1.0,5.0] \mathrm{mol} / \mathrm{l}$.

b Data for $\left[\mathrm{C}_{4} \mathrm{C}_{1} \mathrm{Pyrr}\right]^{+}\left[\mathrm{NTf}_{2}\right]^{-}$in PC solution from ref. 14, linearly interpolated in the range $c \in[0.9,2.5] \mathrm{mol} / \mathrm{l}$.

c Data for $\left[\mathrm{C}_{4} \mathrm{C}_{1} \mathrm{Pyrr}\right]^{+}\left[\mathrm{NTf}_{2}\right]^{-}$in $\mathrm{PC}$ solution from ref. 14, average of data in the range $c \in[2.6,3.4] \mathrm{mol} / \mathrm{l}$.

\section{Ionic liquids confined between like-charged surfaces}

To investigate the influence of confinement on the screening behavior, we conducted additional simulations with $\mathrm{PBC}$ in the $x$ - and $y$-directions only, where the IL is confined between planar, like-charged walls in the $z$-direction as illustrated in Fig. 5 . Depending on the distance between the surfaces, the systems contained between 2000 and 4000 $\left[\mathrm{C}_{4} \mathrm{C}_{1} \mathrm{Im}\right]^{+}\left[\mathrm{NTf}_{2}\right]^{-}$ion pairs (cf. Table II) and an additional number of $352\left[\mathrm{C}_{4} \mathrm{C}_{1} \mathrm{Im}\right]^{+}$counterions compensating the negative wall charge. Note that if we used a force field with full charges, the required number of counterions would be smaller (about 282). Since counterions predominantly accumulate close to the surfaces, in both cases, the solid-liquid interaction should not influence the decay lengths and wavelengths of asymptotic decay modes at large distances $^{30}$ (see Section S2.3 of the supplementary material for a more detailed discussion). In every system, each wall consists of 5376 carbon atoms forming a hexagonal graphene layer with lattice constant $a=0.246 \mathrm{~nm}$. Each carbon atom carried a charge of $-0.02619 e$, resulting in a surface charge density of $\sigma_{q}=-1 e \mathrm{~nm}^{-2}$. For the largest system, we generated four independent replicas. All systems were equilibrated for $300 \mathrm{~ns}$. Each system was simulated for $1 \mu \mathrm{s}$ in production runs, with atom positions stored every $2 \mathrm{ps}$ for a posteriori 
analysis.

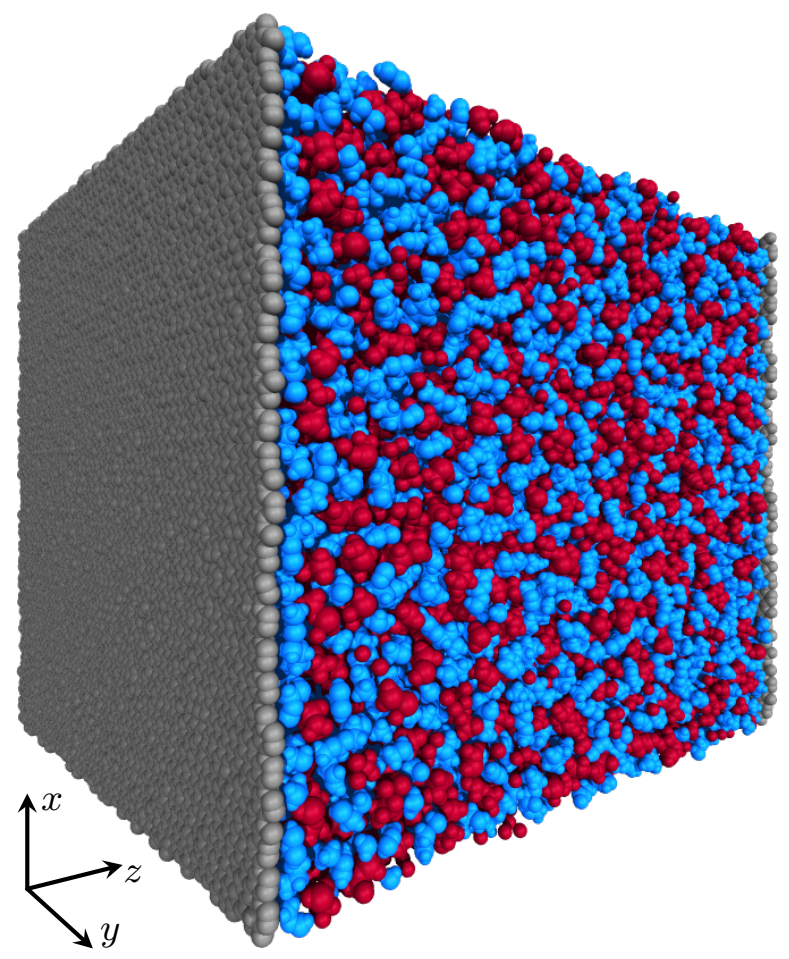

FIG. 5. Example visualization of a confined IL system. The ionic liquid $\left[\mathrm{C}_{4} \mathrm{C}_{1} \mathrm{Im}\right]^{+}\left[\mathrm{NTf}_{2}\right]^{-}$ (cations: blue; anions: red) is confined in the $z$-direction between planar, negatively charged graphene walls (gray).

To investigate the electrostatic screening in these systems, we computed the perpendicular component $E^{\perp}(z)$ of the microscopic electric field in the $z$-direction according to Eq. (10). Since all systems were symmetric in the $z$-direction with respect to their center at $z=L_{z} / 2$, the charge density profiles $\rho_{q}^{\perp}(z)$ were taken as the average of $\rho_{q}^{\perp}(z)$ and $\rho_{q}^{\perp}\left(L_{z}-z\right)$ prior to evaluating Eq. (10) to maximize the statistical quality of the data. The absolute values of the resulting electric fields $\left|E^{\perp}\right|(z)$ are shown in Fig. 6.

After a sharp increase due to the wall charge located at $z \approx 0.355 \mathrm{~nm}$, the electric field remains constant in a thin "empty" slab up to $z \approx 0.6 \mathrm{~nm}$ which exists due to LJ repulsion. Thereafter, because of the high surface charge density of the walls, the IL forms alternating cation-anion layers, resulting in an oscillatory behavior of $\left|E^{\perp}\right|(z)$. Up to $z \approx 1.5 \mathrm{~nm}$, $\left|E^{\perp}\right|(z)$ oscillates with a wavelength that is smaller than the size of the ions. This behavior results from the highly ordered structure of the IL in the vicinity of the walls, where both the rotational and translational motion of the ions is strongly restricted so that their molecular arrangement is almost static. In this arrangement, there exist preferred orientations of the ions with respect to the wall such that certain groups of atoms are predominantly found at specific distances from the wall. Thus, the short oscillation period of the electric field close to the walls is due to intramolecular contributions. A detailed analysis of the contributions of different atom types to the oscillations of the electric field is given in Section S2.2.2 of the supplementary material. With increasing distance from the walls, ionic motion becomes less restricted, so that at distances larger than $z \approx 2 \mathrm{~nm}$, the electric field's oscillations 


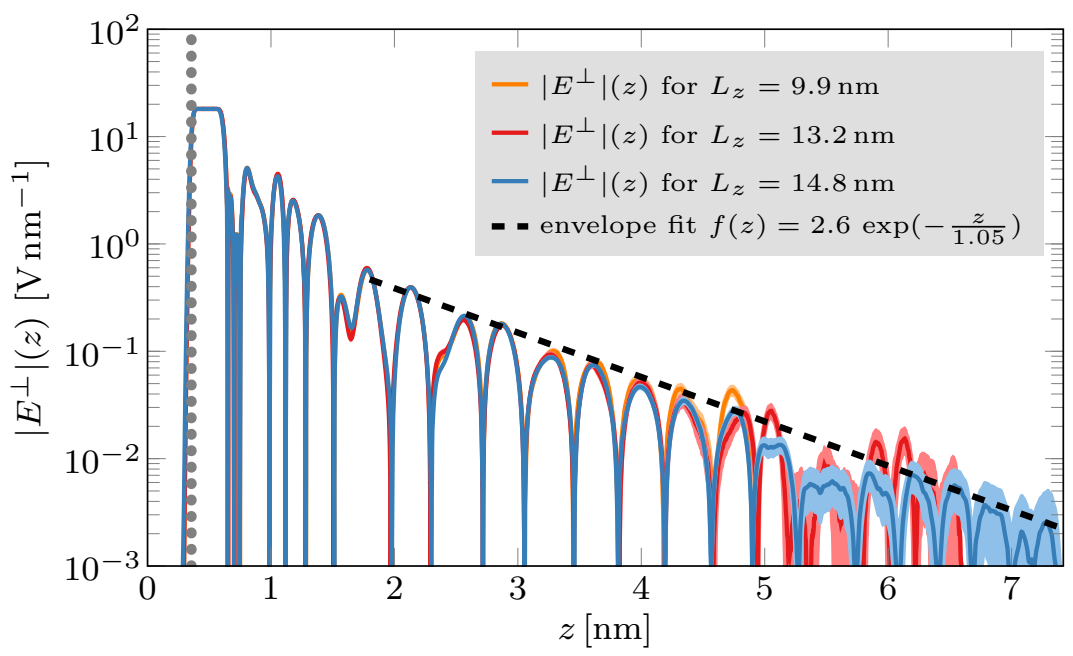

FIG. 6. Absolute value of the electric field's perpendicular component $\left|E^{\perp}\right|(z)$ (log-scale) in $\left[\mathrm{C}_{4} \mathrm{C}_{1} \mathrm{Im}\right]^{+}\left[\mathrm{NTf}_{2}\right]^{-}$confined between like-charged graphene walls. For $z \geq 2 \mathrm{~nm}$, the envelope of $\left|E^{\perp}\right|(z)$ can be described by a function of the form $f(z)=a \exp \left(-z / \lambda_{\mathrm{S}}\right)$ with an amplitude of $a \approx 2.6 \mathrm{~V}$ and a decay length of $\lambda_{\mathrm{S}}=1.05 \mathrm{~nm}$ as indicated by the dashed black line. The dotted gray vertical line marks the average wall position.

smooth out with an almost uniform period of $0.76 \mathrm{~nm}$, which corresponds to approximately two ion diameters and is in good agreement with the thickness $\Delta=0.79 \mathrm{~nm}$ of squeezed-out cation-anion layers measured in SFB experiments using the same $\mathrm{IL}^{35}$. The envelope of the electric field then decreases exponentially with a decay constant of $\lambda=1.05 \mathrm{~nm}$ (dashed black line in Fig. 6), which is in good agreement with the decay length of interionic forces $\lambda_{\mathrm{S}}=1.07 \mathrm{~nm}$ obtained from bulk simulations. The fact that the different lines displayed in Fig. 6 representing $\left|E^{\perp}\right|(z)$ in systems with different inter-wall distances all decay in the same manner suggests that the electrostatic screening behavior is independent of system size.

The electric field, however, reflects only the cumulative average distribution of charges in the system, which might mask other important details. To obtain information about the orientation of ions as a function of their distance from the walls, we computed the average value of the molecular rotational dipole moment's perpendicular component $\left|m_{\text {rot }}^{\perp}\right|(z)$ according to Eq. (12), which is depicted in Fig. 7 for different system sizes. As the molecular dipole moment is a cumulative quantity per molecule, its average cannot contain intramolecular features by construction. In that respect, it is interesting to see that even though up to $z \approx 1 \mathrm{~nm}$, the system consists almost entirely of cations (see Section S2.2.1 of the supplementary material), the first cation layer actually consists of two cation layers with opposite dipolar orientation. At distances exceeding $z=1 \mathrm{~nm}$, the amplitude of the rotational dipole moment's perpendicular component decays in the very same manner as the electric field with a decay constant of $\lambda=1.05 \mathrm{~nm}$. Again, as it was the case for the electric field, the decay of $\left|m_{\text {rot }}^{\perp}\right|(z)$ is the same for all studied system sizes. 


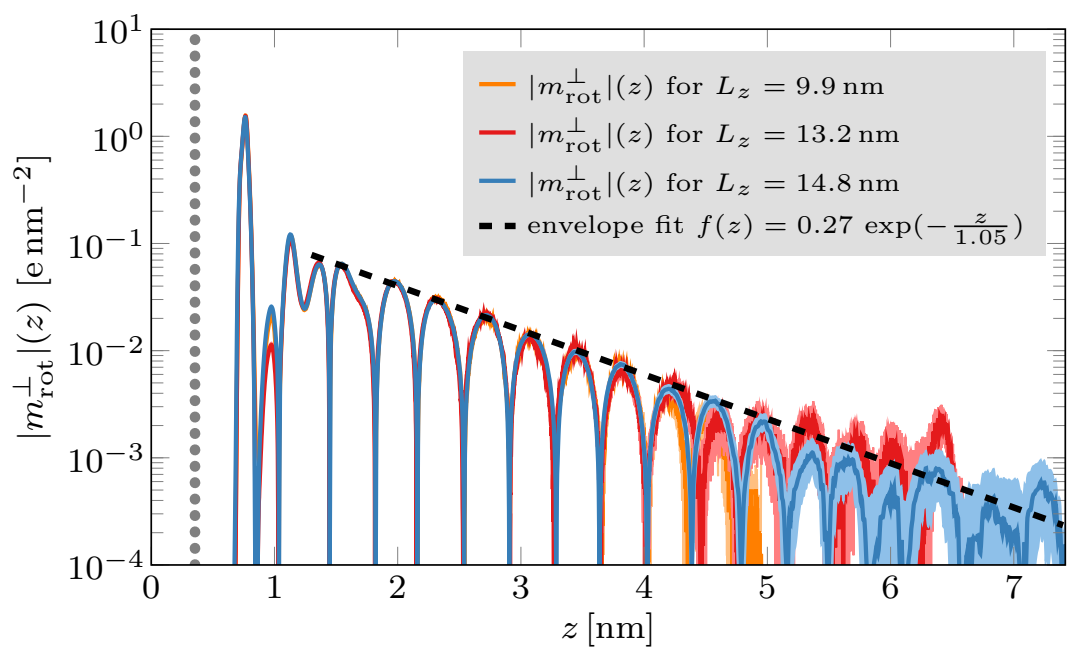

FIG. 7. Absolute value of the perpendicular component $\left|m_{\mathrm{rot}}^{\perp}\right|(z)$ of the rotational dipole moment density (log-scale) in $\left[\mathrm{C}_{4} \mathrm{C}_{1} \mathrm{Im}\right]^{+}\left[\mathrm{NTf}_{2}\right]^{-}$confined between like-charged graphene walls. The envelope of $\left|m_{\text {rot }}^{\perp}\right|(z)$ is depicted by the dashed black line, which again has a decay length of $\lambda_{\mathrm{S}}=1.05 \mathrm{~nm}$ (same as for $\left|E^{\perp}\right|(z)$ ). The average wall position is indicated by the dotted gray vertical line.

\section{Structural decay in confined ionic liquids}

Now that we have analyzed the decay of electrostatic observables in the system, we will have a closer look on the relaxation of the local ionic structure. To this aim, for the largest confined system, we divided the system into $n$ slabs of width $l_{z}$, each centered at a position $z_{i}=z_{0}+i \cdot l_{z}$, where $z_{0}$ is the minimal reference position. We then computed local, short-ranged RDFs $g_{+-}^{(i)}\left(r ; z_{i}\right)$ between cations from slab $i$ and all anions. Even though the system is anisotropic and its extent is bounded in the z-direction, these RDFs are spherical to facilitate their comparison with the cation-anion RDF in the bulk. Of course, this requires the reference positions $z_{i}$ to be located further away from any of the walls than the maximum distance $r_{\text {cut }}$ considered in the RDF computation. We took $n=98$ slabs of width $l_{z}=\frac{L_{z}-2 z_{0}}{n} \approx 0.1 \mathrm{~nm}$, chose the minimum reference position $z_{0}=2.5 \mathrm{~nm}$ (maximum $z_{n}=L_{z}-z_{0}$ ), and evaluated each RDF up to a distance of $r_{\text {cut }}=2 \mathrm{~nm}$. Furthermore, when computing such RDFs, it is important to account for the fluctuating number of cations within each reference slab. Likewise, to obtain comparable results despite the fluctuating number of anions found within a distance $r<r_{\text {cut }}$, all RDFs have been normalized with respect to the bulk anion density of $\approx 2.154 \mathrm{~nm}^{-3}$. Due to the symmetry of the system, we averaged the results of each pair $\left(z_{i}, z_{n-i}\right)$, yielding a total of $\frac{n}{2}=49$ independent RDFs, which are displayed in the top panel of Fig. 8, where the color of the lines change smoothly from the smallest $z_{i}=z_{0}$ (red) to the largest $z_{i}=z_{48}$ (blue). Due to the layered structure of the IL close to the walls, one might expect the shape of those RDFs $g_{+-}^{(i)}\left(r ; z_{i}\right)$ with small $z_{i}$ to be significantly different from those with the reference position close to the center of the system, and that the RDF's inherent spherical averaging may lead to a broadening of the peaks of the ones close to the walls. However, it turns out that the shapes and locations of especially the first maxima and minima of the RDFs are all very similar, and their shapes only differ slightly in the region beyond the second 

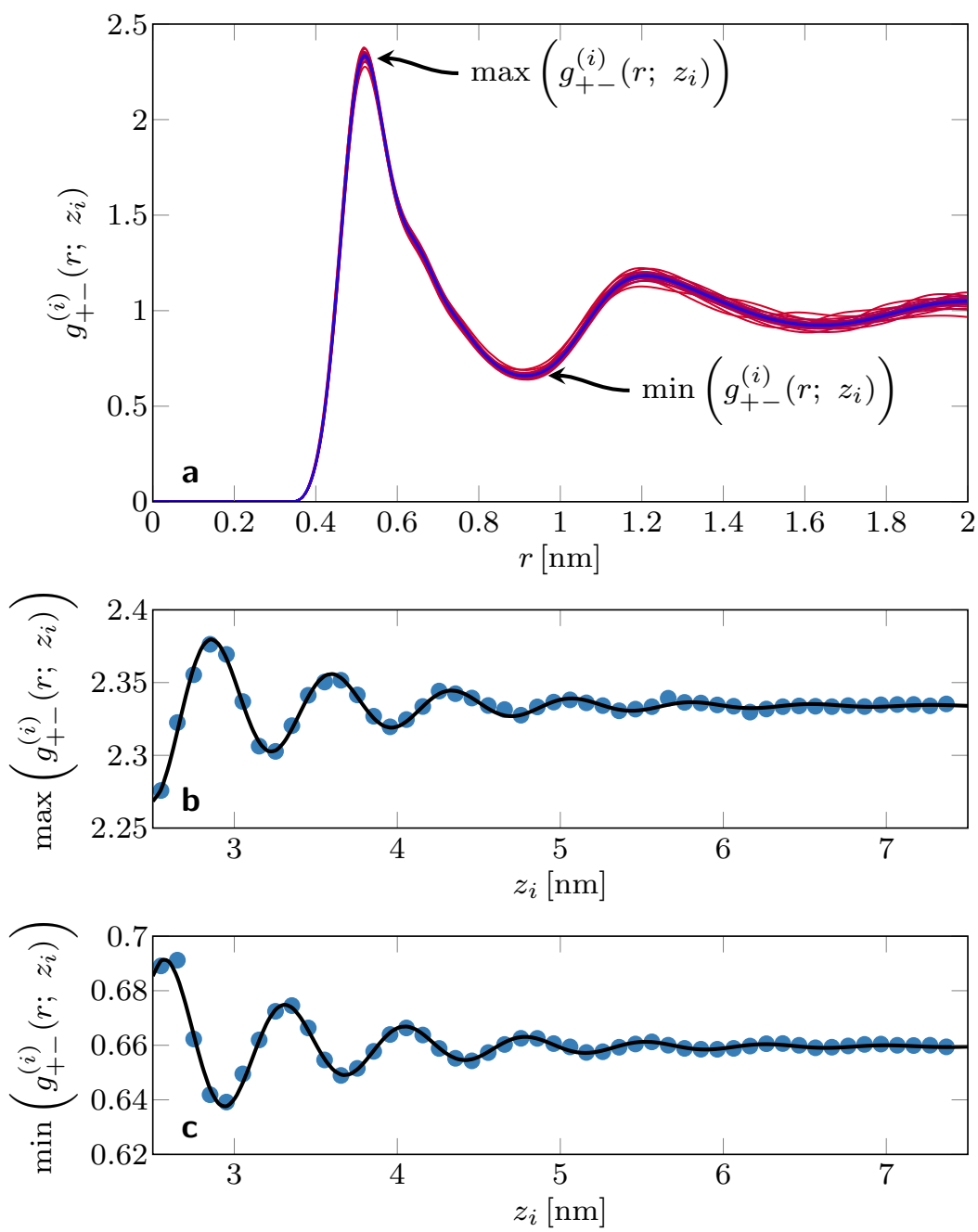

FIG. 8. Panel a: $z$-position-dependent cation-anion RDFs $g_{+-}^{(i)}\left(r ; z_{i}\right)$ of $\left[\mathrm{C}_{4} \mathrm{C}_{1} \mathrm{Im}\right]^{+}\left[\mathrm{NTf}_{2}\right]^{-}$confined between like-charged graphene walls. The lines are RDFs between those cations that have their centers of mass $z_{\text {com }}$ within $2\left|z_{i}-z_{\text {com }}\right| \leq l_{z}\left(z_{i}=z_{0}+i \cdot \Delta z\right.$ with $\left.z_{0}=2.5 \mathrm{~nm}, l_{z} \approx 0.1 \mathrm{~nm}\right)$ and all anions in the system. The color of the lines changes smoothly with the reference position ranging from $z_{i}=z_{0}$ (close to the walls, red) to $z_{i}=z_{48}=\frac{L_{z}}{2}$ (center of the system, blue). Panel $\mathbf{b}$ : Amplitude of the first maximum $\max \left(g_{+-}^{(i)}\left(r ; z_{i}\right)\right)$ of each of the the RDFs depicted in panel $\mathbf{a}$ as a function of the reference distance $z_{i}$ (blue dots). Panel c: Amplitude of the first minimum $\min \left(g_{+-}^{(i)}\left(r ; z_{i}\right)\right)$ of the same RDFs as a function of the reference distance $z_{i}$. The black lines in panels $\mathbf{b}$ and $\mathbf{c}$ are fits of a oscillatory exponential decays with decay length $1 \mathrm{~nm}$. This shows that the local structure around cations relaxes with increasing distance from the walls towards the bulk structure in a manner that is very similar to the oscillatory decay of the electrostatic screening.

maximum. The RDF with the reference group located in the center of the system (the "bluest" line) matches exactly with the cation-anion RDF computed in the bulk system. In the short-range part up to $r \approx 1.2 \mathrm{~nm}$, the only significant deviation can be observed in 
the amplitudes $\max \left(g_{+-}^{(i)}\left(r ; z_{i}\right)\right)$ and $\min \left(g_{+-}^{(i)}\left(r ; z_{i}\right)\right)$ of the first local minima and maxima at $r_{\max } \approx 0.52 \mathrm{~nm}$ and $r_{\min } \approx 0.91 \mathrm{~nm}$, respectively. Because the dependence of these amplitudes on the reference positions is not easily visible in Fig. $8 \mathrm{a}$, we plot $\max \left(g_{+-}^{(i)}\left(r ; z_{i}\right)\right)$ and $\min \left(g_{+-}^{(i)}\left(r ; z_{i}\right)\right)$ versus $z_{i}$ separately in Fig. 8b,c (blue dots). The black lines in these plots are fits of oscillatory exponential decays $f(z)=A \cos \left(\omega z_{i}-\varphi\right) \exp \left(-\frac{z_{i}}{\lambda}\right)+c$ to the data, which yield a decay length $\lambda=1 \mathrm{~nm}$ in both cases. We carried out similar analyses for anion-cation, anion-anion, and cation-cation RDFs, which give a very similar picture with decay lengths between 0.9 and $1.1 \mathrm{~nm}$ (see Section S2.2.3 of the supplementary material). This indicates that with increasing distance from the walls, the (spherically averaged) local arrangement of ions decays to the bulk structure in a very similar manner as the previously analyzed electrostatic properties.

\section{ON THE RELATION OF UNDERSCREENING TO OTHER MEASUREMENTS}

As the long-range monotonic decay of surface interaction forces mediated by concentrated electrolytes and ILs observed in SFB experiments was suggested to reflect the electrostatic screening behavior of these substances, several attempts have been made to relate the measured screening lengths to other observables. ${ }^{18,37}$ Here, we discuss the applicability of some of the theoretical models employed to establish such connections.

\section{A. Differential capacitance}

In ref. 18, Lee et al. suggested that the long-range decay of electrostatic interactions is consistent with concentration-dependent measurements of differential double-layer capacitance. The authors of ref. 18 used an approach similar to the Gouy-Chapman-Stern (GCS) model and divided the electrical double-layer into a Stern and a diffuse layer, so that the total differential capacitance $C_{\mathrm{d}}$ could be modeled as two capacitors in series with capacitance $C_{\text {Stern }}$ and $C_{\text {diffuse }}$, respectively. Unlike $C_{\text {Stern }}$, however, $C_{\text {diffuse }}$ is not constant and depends on the electrostatic potential at the Helmholtz plane separating the diffuse and Stern layers. Assuming low applied potential differences and constant $C_{\text {diffuse }}$, approximated by the capacitance at the potential of zero charge, Lee et al. estimated $C_{\text {diffuse }}=\varepsilon_{0} \varepsilon_{r} / \lambda_{S}$. Note that this expression is the same as the zero-voltage Gouy-Chapman capacitance with $\lambda_{\mathrm{D}}$ replaced by $\lambda_{\mathrm{S}}$. Within these assumptions, Lee et al. obtained for the total capacitance

$$
C_{\mathrm{d}}=\left(C_{\text {Stern }}^{-1}+C_{\text {diffuse }}^{-1}\right)^{-1}=\frac{\varepsilon_{0} \varepsilon_{r}}{a+\lambda_{\mathrm{S}}} .
$$

The concentration dependence of capacitance to compare with experiments comes from that of $\lambda_{\mathrm{S}}$ and $\varepsilon_{r}$.

To compute $C_{\mathrm{d}}$ in our work, we followed the usual practice (see, e.g., ref. 82) and took the width of the Stern layer $a=d / 2$, where $d\left(\approx 0.4 \mathrm{~nm}\right.$ for $\left.\left[\mathrm{C}_{2} \mathrm{C}_{1} \mathrm{Im}\right]^{+}\left[\mathrm{NTf}_{2}\right]^{-}\right)$is the bare ion diameter. Note that in ref. 18 a different value for $a$ was used, which shifts the curves analyzed in the following but does not change the conclusions of this analysis (see Section S3 of the supplementary material for details). Figure 9a compares direct measurements of $C_{\mathrm{d}}$ for $\left[\mathrm{C}_{2} \mathrm{C}_{1} \mathrm{Im}\right]^{+}\left[\mathrm{NTf}_{2}\right]^{-}$solutions in $\mathrm{PC}^{83}$ (black squares) with values obtained 
according to Eq. (15) for similar ILs. For the screening lengths $\lambda_{\mathrm{S}}$ and the relative permittivities $\varepsilon_{r}$ required to evaluate Eq. (15), we used the data either from SFB experiments with $\left[\mathrm{C}_{4} \mathrm{C}_{1} \mathrm{Pyrr}\right]^{+}\left[\mathrm{NTf}_{2}\right]^{-}$in $\mathrm{PC}^{14}$ (orange dots, same as in ref. 18) or from our simulations of $\left[\mathrm{C}_{4} \mathrm{C}_{1} \mathrm{Im}\right]^{+}\left[\mathrm{NTf}_{2}\right]^{-}$in PC (blue dots). Additionally, the green line in Fig. 9a depicts the concentration-dependent behavior of $C_{\mathrm{d}}$ according to the GCS model, i.e., with the screening length $\lambda_{\mathrm{S}}$ in Eq. (15) replaced by the Debye length $\lambda_{\mathrm{D}}$.

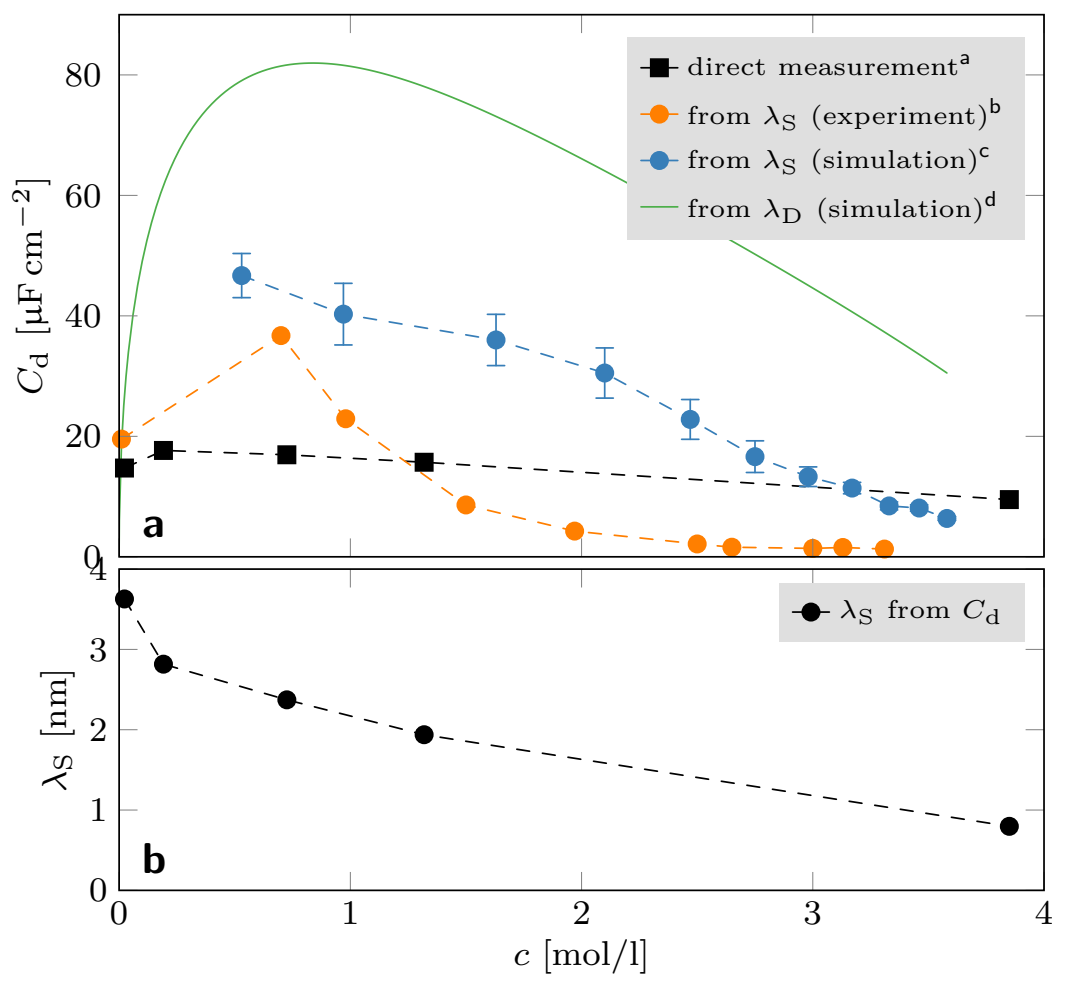

FIG. 9. Panel a: Comparison of the concentration-dependent differential capacitance $C_{\mathrm{d}}$ at the point of zero charge obtained from direct measurements ${ }^{83}$ (black squares) and according to the GCS model as defined by Eq. (15) (orange dots: SFB measurements ${ }^{14}$, blue circles: simulation). The solid green line shows $C_{\mathrm{d}}$ when $\lambda_{\mathrm{S}}$ is replaced by $\lambda_{\mathrm{D}}$ in Eq. (15).

${ }^{a}$ Differential capacitance for $\left[\mathrm{C}_{2} \mathrm{C}_{1} \mathrm{Im}\right]^{+}\left[\mathrm{NTf}_{2}\right]^{-}$from Bozym et al. ${ }^{83}$.

${ }^{\mathrm{b}} \lambda_{\mathrm{S}}$ and $\varepsilon_{r}$ data for $\left[\mathrm{C}_{4} \mathrm{C}_{1} \mathrm{Pyrr}\right]^{+}\left[\mathrm{NTf}_{2}\right]^{-}$from Smith et al. ${ }^{14}$.

${ }^{\mathrm{c}} \lambda_{\mathrm{S}}$ and $\varepsilon_{r}$ data from simulations of $\left[\mathrm{C}_{4} \mathrm{C}_{1} \mathrm{Im}\right]^{+}\left[\mathrm{NTf}_{2}\right]^{-}$(this work).

${ }^{\mathrm{d}} \lambda_{\mathrm{D}}$ computed according to Eq. (1) using interpolated $\varepsilon_{r}$ data from simulations of $\left[\mathrm{C}_{4} \mathrm{C}_{1} \mathrm{Im}\right]^{+}\left[\mathrm{NTf}_{2}\right]^{-}$(this work).

Panel b: Screening length $\lambda_{\mathrm{S}}$ computed according to the inverted GCS model (Eq. (16)) with $C_{\mathrm{d}}$ from direct measurements ${ }^{83}$ and $\varepsilon_{r}$ interpolated from simulations. In both panels, dashed lines serve as a guide to the eye.

Using Eq. (15) with data obtained from simulations appears to yield the best agreement with direct measurements at high concentrations, whereas the SFB-based data fails to reproduce the direct measurements for high concentrations both qualitatively and quantitatively. Interestingly, if $\lambda_{\mathrm{S}}$ is replaced by the Debye length $\lambda_{\mathrm{D}}$ in Eq. (15) (i.e., the GCS model), both the nonmonotonic behavior of $C_{\mathrm{d}}$ and its linear tail are still reproduced quite well 
(green line). Even in this case, despite the quantitative deviation, the qualitative agreement between the GCS model and direct measurements is better than that of Eq. (15) with $\lambda_{\mathrm{S}}$ obtained from SFB experiments. This raises the question whether this approach is capable of establishing any meaningful relation between the screening length and the differential capacitance at high ion concentrations. Furthermore, by solving Eq. (15) for $\lambda_{\mathrm{S}}$, one gets

$$
\lambda_{\mathrm{S}}=\frac{\varepsilon_{0} \varepsilon_{r}}{C_{\mathrm{d}}}-a .
$$

By evaluating Eq. (16) for the directly measured values of $C_{\mathrm{d}}$, we obtained the corresponding 'prediction' for $\lambda_{\mathrm{S}}$, which is depicted in Fig. 9b. The observed monotonic decrease of $\lambda_{\mathrm{S}}$ with increasing concentration demonstrates that Eq. (15) is unable to reproduce the expected nonmononic behavior of $\lambda_{\mathrm{S}}$, which, in turn, leads to the conclusion that this model cannot be used to connect the measured $C_{\mathrm{d}}$ values with $\lambda_{\mathrm{S}}$ at high concentrations.

\section{B. Excess chemical potential}

Another interesting approach to relating bulk electrolyte properties to $\lambda_{\mathrm{S}}$ was presented in ref. 37, where the screening length of aqueous $\mathrm{NaCl}$ solutions obtained from SFB measurements was used to predict the excess chemical potential $\mu_{\text {ex }}$ of the solution according to

$$
\mu_{\mathrm{ex}}=-\frac{1}{2} \frac{l_{B} k_{\mathrm{B}} T}{\lambda_{\mathrm{S}}+d}=-\frac{z^{2} e^{2}}{8 \pi \varepsilon_{0} \varepsilon_{r}\left(\lambda_{\mathrm{S}}+d\right)},
$$

where $l_{\mathrm{B}}$ denotes the Bjerrum length (see ref. 18 for a derivation). Note that this expression is identical to what one would obtain from Debye-Hückel theory but with the effective screening length $\lambda_{\mathrm{S}}$ replacing the Debye length $\lambda_{\mathrm{D}} \cdot{ }^{18}$ In Fig. 10a, the resulting prediction of $\mu_{\mathrm{ex}}$ for $\lambda_{\mathrm{S}}$ obtained from SFB experiments ${ }^{14}$ is compared with $\mu_{\mathrm{ex}}$ obtained from chemical activity coefficients ${ }^{84} \gamma$ according to $\mu_{\mathrm{ex}}=k_{\mathrm{B}} T \ln (\gamma)$.

While the quantitative agreement of both data series in Fig. 10a is indeed remarkable, the qualitative behavior at intermediate to high concentrations appears to be different. To investigate this discrepancy, we invert the model to calculate $\lambda_{\mathrm{S}}$ from $\mu_{\mathrm{ex}}$ as

$$
\lambda_{\mathrm{S}}=-\frac{z^{2} e^{2}}{8 \pi \varepsilon_{0} \varepsilon_{r} \mu_{\mathrm{ex}}}-d
$$

which allows us to conduct a scaling analysis in terms of the behavior of $\lambda_{S} / \lambda_{D}$ with respect to $d / \lambda_{\mathrm{D}}$ (similar to that in Section III B) for both data series. The resulting scalings are shown in Fig. 10b. As expected, the screening length $\lambda_{\mathrm{S}}$ from SFB measurements exhibits the cubic scaling $\lambda_{\mathrm{S}} / \lambda_{\mathrm{D}} \propto\left(d / \lambda_{\mathrm{D}}\right)^{3}$ (orange line) found for the decay of surface forces in many SFB measurements of concentrated electrolytes ${ }^{14,15,18,37}$. In contrast, the scaling of the screening length computed according to Eq. (18) (black dots) can be well described by a phenomenological function of the form $f\left(d / \lambda_{\mathrm{D}}\right)=a_{0}+a_{1}\left(a_{2}-d / \lambda_{\mathrm{D}}\right)^{\alpha}$ (solid black line), which shows a qualitatively entirely different behavior. Since $\alpha<0$, it diverges for $d / \lambda_{\mathrm{D}} \rightarrow a_{2}$, which corresponds to a diverging screening length $\lambda_{\mathrm{S}}$ for $\mu_{e x} \rightarrow 0$ in Eq. (18) as the concentration approaches the solubility threshold. However, such a behavior seems unphysical, as it would imply that free ions in a saturated solution were unable to respond to an external electric field.

Thus, as the model fails at high concentrations, it is questionable whether the mere quantitative agreement of the data in Fig. 10a in this regime allows drawing any meaningful conclusions. 

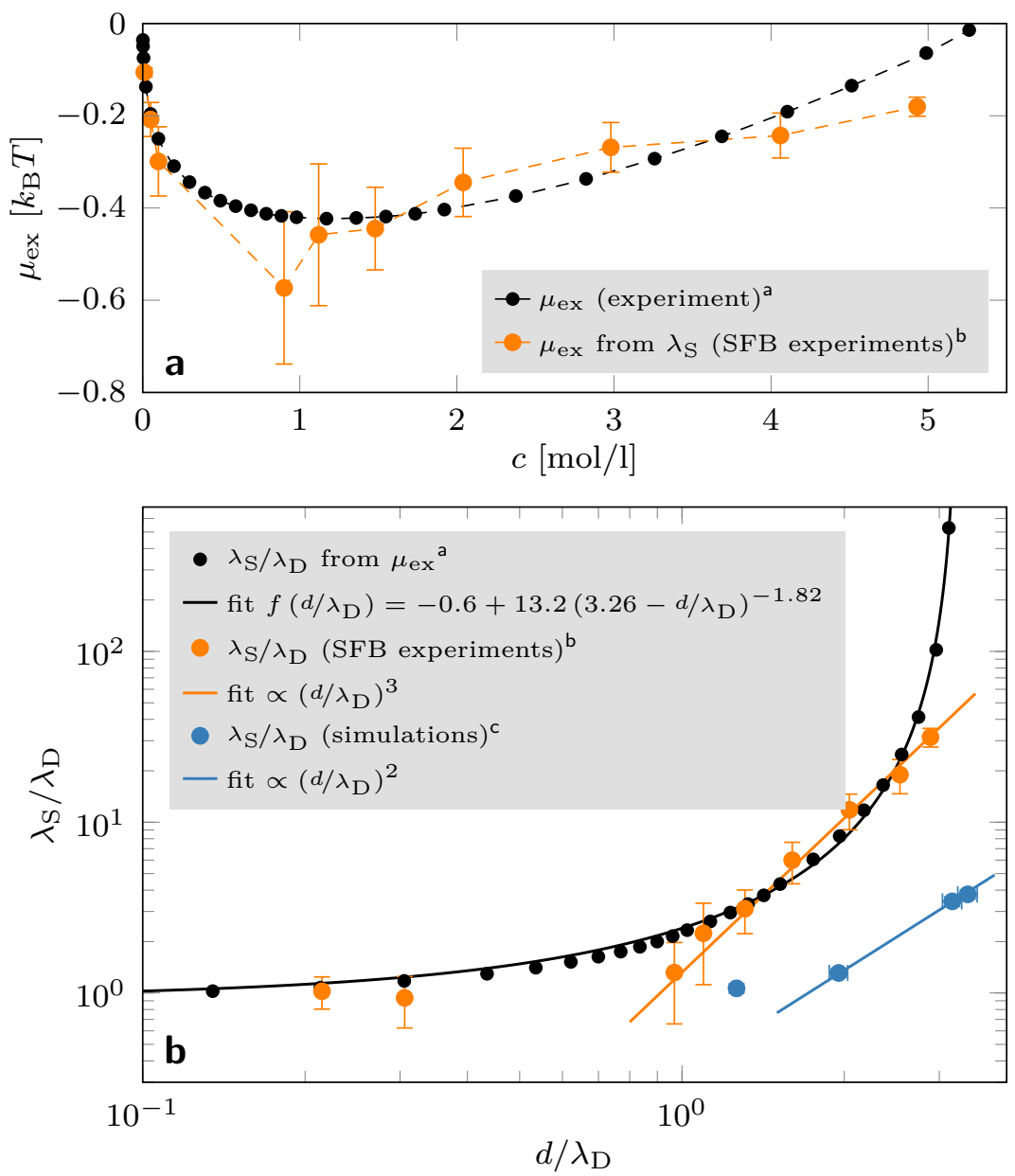

FIG. 10. Panel a: Excess chemical potential $\mu_{\mathrm{ex}}$ of aqueous $\mathrm{NaCl}$ solutions computed from activity coefficients $^{84} \gamma$ according to $\mu_{e x}=k_{\mathrm{B}} T \ln (\gamma)$ (black dots) and computed via Eq. (18) with $\lambda_{\mathrm{S}}$ taken from SFB measurements ${ }^{14}$ (orange dots). Dashed lines serve as guides to the eye.

Panel b: Comparison of the scaling $\lambda_{\mathrm{S}} / \lambda_{\mathrm{D}}$ versus $d / \lambda_{\mathrm{D}}$ for $\lambda_{\mathrm{S}}$ obtained from $\mu_{\mathrm{ex}}$ of aqueous $\mathrm{NaCl}$ solutions according to Eq. (18) (black dots), for $\lambda_{\mathrm{S}}$ obtained from corresponding SFB experiments (orange dots), and for $\lambda_{\mathrm{S}}$ from bulk simulations (blue dots). The solid black line represents a phenomenological fit of the form $f(x)=a_{0}+a_{1}\left(a_{2}-x\right)^{\alpha}$ with $x:=d / \lambda_{\mathrm{D}}$.

${ }^{a}$ Activity coefficients $\gamma$ from ref. 84 to obtain $\mu_{e x}=k_{\mathrm{B}} T \ln (\gamma)$.

${ }^{\mathrm{b}}$ Screening lengths $\lambda_{\mathrm{S}}$ and permittivities $\varepsilon_{r}$ (to compute $\lambda_{\mathrm{D}}$ ) from ref. 14 .

\section{CONCLUSION}

We have conducted large-scale MD simulations of concentrated electrolytes and pure ionic liquids in bulk and under confinement and calculated the PMFs between different ionic species with unprecedented precision. The large system sizes and long simulation times allowed us to analyze the PMFs in a range of interionic distances corresponding to the range of surface separations in SFB experiments. In contrast to experimental measurements, we found no evidence for the existence of a long-ranged, monotonic decay of effective interionic 
interactions. We obtained the characteristic decay lengths $\lambda_{\mathrm{S}}$ of all analyzed PMFs about an order of magnitude smaller than those inferred from the experiments.

We investigated the concentration-dependent scaling of the effective screening lengths $\lambda_{\mathrm{S}}$ with respect to the Debye length $\lambda_{\mathrm{D}}$ by analyzing interionic PMFs in electrolyte solutions for different ion concentrations. We found that there are at least two different decay lengths in the PMFs, with the asymptotic scaling $\lambda_{\mathrm{S}} / \lambda_{\mathrm{D}} \propto\left(d / \lambda_{\mathrm{D}}\right)^{2}$ prevailing at high ion concentrations. We recall that this scaling has also been found theoretically by Adar et al. ${ }^{38}$ and similar to our earlier results ${ }^{43}$ in simulations by Krucker-Velasquez and Swan ${ }^{81}$. However, it contrasts with the experimentally observed scaling $\lambda_{\mathrm{S}} / \lambda_{\mathrm{D}} \propto\left(d / \lambda_{\mathrm{D}}\right)^{3}$ of the decay of forces between charged surfaces across concentrated electrolytes.

Our results for IL systems confined between like-charged surfaces did not show any anomalously long electrostatic decay lengths either, confirming our findings from bulk simulations. The determined values of the screening lengths are in close agreement with the values obtained for the same systems in bulk, confirming the statistical-mechanical finding that solidliquid interactions do not affect asymptotic decay lengths. ${ }^{30}$ Our values compare well with the results of other studies based on smaller all-atom MD simulations ${ }^{85}$ as well as with theoretical approaches and simulations of primitive model electrolytes ${ }^{38,86,87}$.

We showed that relating screening lengths in highly concentrated electrolytes to other observables may be of limited value if the employed models fail at high ion concentrations and may therefore yield unphysical predictions in this regime. This highlights the importance of the ongoing efforts in the research on highly concentrated electrolytes and ILs. The physics of such systems remain an intricate subject, and there exist many possible approaches aiming to capture the existence of long-ranged monotonic decays (see, e.g., ref. 15 for an overview). Nevertheless, our simulation results indicate that the experimentally observed long-ranged monotonic decay of surface forces might not be a property of bulk electrolytes or ILs in the bulk and in confinement.

As of now, the origin of these forces is not understood. However, from a computational perspective, we see several possible directions for future research. To strengthen the link between simulation studies and analytical descriptions of concentrated electrolytes, the work of Kjellander on dressed ion theory ${ }^{17,30,32,41,42,88}$ appears as a prime candidate not only because of its comprehensiveness, but also due to its direct applicability to MD simulation data. As it directly relates an electrolyte's short-range screening behavior to its corresponding asymptotic decay modes, it would be particularly interesting to follow the route Kjellander outlined in ref. 17 (demonstrated for spherical ions in ref. 89) to extract such modes from our high-precision data.

Although the reduced-charge IL models used in this work provide the correct dynamics, such models influence the Coulomb interactions uniformly among all kinds of particles in a system at all distances. In this respect, another interesting question is how the electronic polarizability of ions affects the long-range electrostatic screening. However, since underscreening has been observed experimentally for various electrolytes with very different ionic polarizabilities, we do not expect electronic polarizability to play a significant role in longrange electrostatic screening. Nevertheless, models taking electronic polarizability explicitly into account should reproduce both short- and long-range Coulomb interactions more accurately. It might thus be worthwhile investigating its influence in MD simulations employing polarizable force fields.

Furthermore, with respect to SFB experiments, simulations with static system sizes might be unable to capture dynamic processes that could be important to reproduce and explain the 
experimental observations. Lhermerout and Perkin ${ }^{90}$ and also Han and Espinosa-Marzal ${ }^{35}$ have used classical hydrodynamic models to disentangle electrostatic and viscous forces acting during the squeeze-out of ion layers of ILs confined between the mica-coated surfaces of a surface force apparatus. However, the electrostatic screening lengths required to fit the models to the experimental data were still in the same range that had been previously reported without taking fluid viscosity into account. Providing a simulational backing for these approaches in terms of a dynamic microscopic picture will be challenging, as both the spatial and temporal scales of the experimental procedure are not easily accessible by simulations with molecular resolution. Concerning MD simulations, possible steps towards a more realistic picture with a closer correspondence to the experimental setup might include simulations of confined systems where the lateral pressure component in the center of the system is held constant, simulations at constant chemical potential, or even combinations thereof with dynamically changing confinement.

\section{SUPPLEMENTARY MATERIAL}

The supplementary material contains details about numerical methods and error estimation, additional results and analyses, and simulation parameters.

\section{ACKNOWLEDGMENTS}

We thank R. Kjellander for inspiring discussions during his visit to the ICP. S.K. also thanks A. Ciach and O. Patsahan for discussions regarding ref. 39. We acknowledge support by the High Performance and Cloud Computing Group at the Zentrum für Datenverarbeitung of the University of Tübingen, the state of Baden-Württemberg through bwHPC and the German Research Foundation (DFG) through grant no INST 37/935-1 FUGG. Furthermore, we are grateful for computing resources on the Cray XC40 (Hazel Hen) cluster provided by the High Performance Computing Center Stuttgart (HLRS), Germany. C. H. acknowledges financial contributions from the DFG (German Research Foundation) under Germanys Excellence Strategy EXC 2075390740016 and grants CRC 1313-Project-ID 327154368 and CRC 1333-Project-ID 358283783.

\section{AUTHOR DECLARATIONS}

The authors have no conflicts to disclose.

\section{DATA AVAILABILITY}

The data that support the findings of this study are available from the corresponding author upon reasonable request.

[1] R. D. Rogers and K. R. Seddon, Ionic liquids-solvents of the future?, Science 302, 792 (2003). 
[2] S. Pandey, Analytical applications of room-temperature ionic liquids: A review of recent efforts, Analytica Chimica Acta 556, 38 (2006).

[3] R. Hayes, G. G. Warr, and R. Atkin, Structure and nanostructure in ionic liquids, Chemical Reviews 115, 6357 (2015).

[4] Z. Lei, B. Chen, Y.-M. Koo, and D. R. MacFarlane, Introduction: Ionic liquids, Chemical Reviews 117, 6633 (2017).

[5] A. E. Visser, R. P. Swatloski, W. M. Reichert, R. Mayton, S. Sheff, A. Wierzbicki, J. H. Davis Jr., and R. D. Rogers, Task-specific ionic liquids for the extraction of metal ions from aqueous solutions, Chemical Communications , 135 (2001).

[6] T. L. Greaves, A. Weerawardena, C. Fong, I. Krodkiewska, and C. J. Drummond, Protic ionic liquids: Solvents with tunable phase behavior and physicochemical properties, The Journal of Physical Chemistry B 110, 22479 (2006).

[7] T. L. Greaves, A. Weerawardena, C. Fong, I. Krodkiewska, and C. J. Drummond, Correction of "protic ionic liquids: Solvents with tunable phase behavior and physicochemical properties", The Journal of Physical Chemistry B 110, 26506 (2006).

[8] V. Lesch, A. Heuer, V. A. Tatsis, C. Holm, and J. Smiatek, Peptides in the presence of aqueous ionic liquids: tunable co-solutes as denaturants or protectants?, Physical Chemistry Chemical Physics 17, 26049 (2015).

[9] M. A. Gebbie, M. Valtiner, X. Banquy, E. T. Fox, W. A. Henderson, and J. N. Israelachvili, Ionic liquids behave as dilute electrolyte solutions, Proceedings of the National Academy of Sciences of the United States of America 110, 9674 (2013).

[10] T. Baimpos, B. R. Shrestha, S. Raman, and M. Valtiner, Effect of interfacial ion structuring on range and magnitude of electric double layer, hydration, and adhesive interactions between mica surfaces in 0.05-3 $\mathrm{M} \mathrm{Li}^{+}$and $\mathrm{Cs}^{+}$electrolyte solutions, Langmuir 30, 4322 (2014).

[11] R. M. Espinosa-Marzal, A. Arcifa, A. Rossi, and N. D. Spencer, Microslips to "avalanches" in confined, molecular layers of ionic liquids, The Journal of Physical Chemistry Letters 5, 179 (2014).

[12] H.-W. Cheng, P. Stock, B. Moeremans, T. Baimpos, X. Banquy, F. U. Renner, and M. Valtiner, Characterizing the influence of water on charging and layering at electrified ionic-liquid/solid interfaces, Advanced Materials Interfaces 2, 1500159 (2015).

[13] M. A. Gebbie, H. A. Dobbs, M. Valtiner, and J. N. Israelachvili, Long-range electrostatic screening in ionic liquids, Proceedings of the National Academy of Sciences of the United States of America 112, 7432 (2015).

[14] A. M. Smith, A. A. Lee, and S. Perkin, The electrostatic screening length in concentrated electrolytes increases with concentration, The Journal of Physical Chemistry Letters 7, 2157 (2016).

[15] M. A. Gebbie, A. M. Smith, H. A. Dobbs, A. A. Lee, G. G. Warr, X. Banquy, M. Valtiner, M. W. Rutland, J. N. Israelachvili, S. Perkin, and R. Atkin, Long range electrostatic forces in ionic liquids, Chemical Communications 53, 1214 (2017).

[16] R. J. F. Leote de Carvalho and R. Evans, The decay of correlations in ionic fluids, Molecular Physics 83, 619 (1994).

[17] R. Kjellander, The intimate relationship between the dielectric response and the decay of intermolecular correlations and surface forces in electrolytes, Soft Matter 15, 5866 (2019).

[18] A. A. Lee, C. S. Perez-Martinez, A. M. Smith, and S. Perkin, Underscreening in concentrated electrolytes, Faraday Discussions 199, 239 (2017). 
[19] P. Debye and E. Hückel, Zur Theorie der Elektrolyte. I. Gefrierpunktserniedrigung und verwandte Erscheinungen, Physikalische Zeitschrift 24, 185 (1923).

[20] M. Gouy, Sur la constitution de la charge électrique à la surface d'un électrolyte, Journal de Physique Théorique et Appliquée 9, 457 (1910).

[21] D. L. Chapman, A contribution to the theory of electrocapillarity, Philosophical Magazine 25, 475 (1913).

[22] P. Attard, Asymptotic analysis of primitive model electrolytes and the electrical double layer, Physical Review E 48, 3604 (1993).

[23] R. Evans, R. J. F. Leote de Carvalho, J. R. Henderson, and D. C. Hoyle, Asymptotic decay of correlations in liquids and their mixtures, The Journal of Chemical Physics 100, 591 (1994).

[24] L. M. Varela, M. García, and V. Mosquera, Exact mean-field theory of ionic solutions: nonDebye screening, Physics Reports 382, 1 (2003).

[25] A. A. Kornyshev, Double-layer in ionic liquids: Paradigm change?, The Journal of Physical Chemistry B 111, 5545 (2007).

[26] M. A. Brown, G. V. Bossa, and S. May, Emergence of a Stern layer from the incorporation of hydration interactions into the Gouy-Chapman model of the electrical double layer, Langmuir 31, 11477 (2015).

[27] Z. A. H. Goodwin, G. Feng, and A. A. Kornyshev, Mean-field theory of electrical double layer in ionic liquids with account of short-range correlations, Electrochimica Acta 225, 190 (2017).

[28] F. Coupette, A. A. Lee, and A. Härtel, Screening lengths in ionic fluids, Physical Review Letters 121, 075501 (2018).

[29] N. Gavish, D. Elad, and A. Yochelis, From solvent-free to dilute electrolytes: Essential components for a continuum theory, The Journal of Physical Chemistry Letters 9, 36 (2018).

[30] R. Kjellander, Focus article: Oscillatory and long-range monotonic exponential decays of electrostatic interactions in ionic liquids and other electrolytes: The significance of dielectric permittivity and renormalized charges, The Journal of Chemical Physics 148, 193701 (2018).

[31] B. Rotenberg, O. Bernard, and J.-P. Hansen, Underscreening in ionic liquids: a first principles analysis, Journal of Physics: Condensed Matter 30, 054005 (2018).

[32] R. Kjellander, A multiple decay-length extension of the Debye-Hückel theory: to achieve high accuracy also for concentrated solutions and explain under-screening in dilute symmetric electrolytes, Physical Chemistry Chemical Physics 22, 23952 (2020).

[33] P. Gaddam and W. Ducker, Electrostatic screening length in concentrated salt solutions, Langmuir 35, 5719 (2019).

[34] N. Hjalmarsson, R. Atkin, and M. W. Rutland, Switchable long-range double layer force observed in a protic ionic liquid, Chemical Communications 53, 647 (2017).

[35] M. Han and R. M. Espinosa-Marzal, Electroviscous retardation of the squeeze out of nanoconfined ionic liquids, The Journal of Physical Chemistry C 122, 21344 (2018).

[36] M. Han and R. M. Espinosa-Marzal, Influence of water on structure, dynamics, and electrostatics of hydrophilic and hydrophobic ionic liquids in charged and hydrophilic confinement between mica surfaces, ACS Applied Materials \& Interfaces 11, 33465 (2019).

[37] A. A. Lee, C. S. Perez-Martinez, A. M. Smith, and S. Perkin, Scaling analysis of the screening length in concentrated electrolytes, Physical Review Letters 119, 026002 (2017).

[38] R. M. Adar, S. A. Safran, H. Diamant, and D. Andelman, Screening length for finite-size ions in concentrated electrolytes, Physical Review E 100, 042615 (2019).

[39] A. Ciach and O. Patsahan, Correct scaling of the correlation length from a theory for concentrated electrolytes, Journal of Physics: Condensed Matter 33, 37LT01 (2021). 
[40] J. R. Rumble, ed., CRC Handbook of Chemistry and Physics, 100th ed., CRC Handbook of Chemistry and Physics (CRC Press / Taylor \& Francis, Boca Raton, Florida, 2019).

[41] R. Kjellander and D. J. Mitchell, An exact but linear and Poisson-Boltzmann-like theory for electrolytes and colloid dispersions in the primitive model, Chemical Physics Letters 200, 76 (1992).

[42] R. Kjellander and D. J. Mitchell, Dressed-ion theory for electrolyte solutions: A Debye-Hückellike reformulation of the exact theory for the primitive model, The Journal of Chemical Physics 101, 603 (1994).

[43] J. Zeman, S. Kondrat, and C. Holm, Bulk ionic screening lengths from extremely large-scale molecular dynamics simulations, Chemical Communications 56, 15635 (2020).

[44] W. Humphrey, A. Dalke, and K. Schulten, VMD: Visual molecular dynamics, Journal of Molecular Graphics 14, 33 (1996).

[45] M. J. Abraham, T. Murtola, R. Schulz, S. Páll, J. C. Smith, B. Hess, and E. Lindahl, GROMACS: High performance molecular simulations through multi-level parallelism from laptops to supercomputers, SoftwareX 1, 19 (2015).

[46] S. Páll, M. J. Abraham, C. Kutzner, B. Hess, and E. Lindahl, Tackling exascale software challenges in molecular dynamics simulations with GROMACS, in Solving Software Challenges for Exascale: International Conference on Exascale Applications and Software, EASC 2014, Stockholm, Sweden, April 2-3, 2014, Revised Selected Papers, edited by S. Markidis and E. Laure (Springer International, 2015) pp. 3-27.

[47] S. Pronk, S. Páll, R. Schulz, P. Larsson, P. Bjelkmar, R. Apostolov, M. R. Shirts, J. C. Smith, P. M. Kasson, D. van der Spoel, B. Hess, and E. Lindahl, Gromacs 4.5: a high-throughput and highly parallel open source molecular simulation toolkit, Bioinformatics 29, 845 (2013).

[48] B. Hess, C. Kutzner, D. van der Spoel, and E. Lindahl, GROMACS 4: Algorithms for highly efficient, load-balanced, and scalable molecular simulation, Journal of Chemical Theory and Computation 4, 435 (2008).

[49] D. van der Spoel, E. Lindahl, B. Hess, G. Groenhof, A. E. Mark, and H. J. C. Berendsen, GROMACS: Fast, flexible, and free, Journal of Computational Chemistry 26, 1701 (2005).

[50] E. Lindahl, B. Hess, and D. van der Spoel, GROMACS 3.0: A package for molecular simulation and trajectory analysis, Journal of Molecular Modeling 7, 306 (2001).

[51] H. J. C. Berendsen, D. van der Spoel, and R. van Drunen, GROMACS: A message-passing parallel molecular dynamics implementation, Computer Physics Communications 91, 43 (1995).

[52] U. Essmann, L. Perera, M. L. Berkowitz, T. Darden, H. Lee, and L. G. Pedersen, A smooth Particle Mesh Ewald method, The Journal of Chemical Physics 103, 8577 (1995).

[53] C. L. Wennberg, T. Murtola, B. Hess, and E. Lindahl, Lennard-Jones lattice summation in bilayer simulations has critical effects on surface tension and lipid properties, Journal of Chemical Theory and Computation 9, 3527 (2013).

[54] I.-C. Yeh and M. L. Berkowitz, Ewald summation for systems with slab geometry, The Journal of Chemical Physics 111, 3155 (1999).

[55] B. Doherty, X. Zhong, S. Gathiaka, B. Li, and O. Acevedo, Revisiting OPLS force field parameters for ionic liquid simulations, Journal of Chemical Theory and Computation 13, $6131(2017)$.

[56] D. Roy and M. Maroncelli, An improved four-site ionic liquid model, The Journal of Physical Chemistry B 114, 12629 (2010).

[57] I. Leontyev and A. Stuchebrukhov, Accounting for electronic polarization in non-polarizable force fields, Physical Chemistry Chemical Physics 13, 2613 (2011). 
[58] F. Dommert, K. Wendler, R. Berger, L. D. Site, and C. Holm, Force fields for studying the structure and dynamics of ionic liquids: A critical review of recent developments, ChemPhysChem 13, 1625 (2012).

[59] J. Rigby and E. I. Izgorodina, Assessment of atomic partial charge schemes for polarisation and charge transfer effects in ionic liquids, Physical Chemistry Chemical Physics 15, 1632 (2013).

[60] I. V. Leontyev and A. A. Stuchebrukhov, Electronic continuum model for molecular dynamics simulations, The Journal of Chemical Physics 130, 085102 (2009).

[61] F. Dommert, J. Schmidt, C. Krekeler, Y. Y. Zhao, R. Berger, L. Delle Site, and C. Holm, Towards multiscale modeling of ionic liquids: From electronic structure to bulk properties, Journal of Molecular Liquids 152, 2 (2010).

[62] J. Schmidt, C. Krekeler, F. Dommert, Y. Zhao, R. Berger, L. Delle Site, and C. Holm, Ionic charge reduction and atomic partial charges from first-principles calculations of 1,3dimethylimidazolium chloride, The Journal of Physical Chemistry B 114, 6150 (2010).

[63] K. Wendler, S. Zahn, F. Dommert, R. Berger, C. Holm, B. Kirchner, and L. D. Site, Locality and fluctuations: Trends in imidazolium-based ionic liquids and beyond, Journal of Chemical Theory and Computation 7, 3040 (2011).

[64] K. Wendler, F. Dommert, Y. Zhao, R. Berger, C. Holm, and L. Delle Site, Ionic liquids studied across different scales: A computational perspective, Faraday Discussions 154, 111 (2012).

[65] J. Zeman, F. Uhlig, J. Smiatek, and C. Holm, A coarse-grained polarizable force field for the ionic liquid 1-butyl-3-methylimidazolium hexafluorophosphate, Journal of Physics: Condensed Matter 29, 504004 (2017).

[66] F. Uhlig, J. Zeman, J. Smiatek, and C. Holm, First-principles parametrization of polarizable coarse-grained force fields for ionic liquids, Journal of Chemical Theory and Computation 14, 1471 (2018).

[67] S. Weerasinghe and P. E. Smith, A Kirkwood-Buff derived force field for sodium chloride in water, The Journal of Chemical Physics 119, 11342 (2003).

[68] H. J. C. Berendsen, J. R. Grigera, and T. P. Straatsma, The missing term in effective pair potentials, The Journal of Physical Chemistry 91, 6269 (1987).

[69] M. Takeuchi, Y. Kameda, Y. Umebayashi, S. Ogawa, T. Sonoda, S.-i. Ishiguro, M. Fujita, and M. Sano, Ion-ion interactions of $\mathrm{LiPF}_{6}$ and $\mathrm{LiBF}_{4}$ in propylene carbonate solutions, Journal of Molecular Liquids 148, 99 (2009).

[70] N. Michaud-Agrawal, E. J. Denning, T. B. Woolf, and O. Beckstein, MDAnalysis: A toolkit for the analysis of molecular dynamics simulations, Journal of Computational Chemistry 32, 2319 (2011).

[71] R. J. Gowers, M. Linke, J. Barnoud, T. J. E. Reddy, M. N. Melo, S. L. Seyler, J. Domański, D. L. Dotson, S. Buchoux, I. M. Kenney, and O. Beckstein, MDAnalysis: A Python package for the rapid analysis of molecular dynamics simulations, in Proceedings of the 15th Python in Science Conference, edited by S. Benthall and S. Rostrup (2016) pp. 98-105.

[72] L. D. Dalcín, R. R. Paz, and M. Storti, MPI for Python, Journal of Parallel and Distributed Computing 65, 1108 (2005).

[73] L. D. Dalcín, R. R. Paz, M. Storti, and J. D'Elía, MPI for Python: Performance improvements and MPI-2 extensions, Journal of Parallel and Distributed Computing 68, 655 (2008).

[74] L. D. Dalcín, R. R. Paz, P. A. Kler, and A. Cosimo, Parallel distributed computing using Python, Advances in Water Resources 34, 1124 (2011). 
[75] M. Neumann and O. Steinhauser, On the calculation of the frequency-dependent dielectric constant in computer simulations, Chemical Physics Letters 102, 508 (1983).

[76] M. Sega, S. S. Kantorovich, A. Arnold, and C. Holm, On the calculation of the dielectric properties of liquid ionic systems, in Recent Advances in Broadband Dielectric Spectroscopy, NATO Science for Peace and Security Series B: Physics and Biophysics, edited by Y. P. Kalmykov (Springer Netherlands, 2013) pp. 103-122.

[77] C. Schröder, M. Haberler, and O. Steinhauser, On the computation and contribution of conductivity in molecular ionic liquids, The Journal of Chemical Physics 128, 134501 (2008).

[78] S. Gabl, C. Schröder, and O. Steinhauser, Computational studies of ionic liquids: Size does matter and time too, The Journal of Chemical Physics 137, 094501 (2012).

[79] J.-F. Côté, D. Brouillette, J. E. Desnoyers, J.-F. Rouleau, J.-M. St-Arnaud, and G. Perron, Dielectric constants of acetonitrile, $\gamma$-butyrolactone, propylene carbonate, and 1,2dimethoxyethane as a function of pressure and temperature, Journal of Solution Chemistry 25, 1163 (1996).

[80] C. Daguenet, P. J. Dyson, I. Krossing, A. Oleinikova, J. Slattery, C. Wakai, and H. Weingärtner, Dielectric response of imidazolium-based room-temperature ionic liquids, The Journal of Physical Chemistry B 110, 12682 (2006).

[81] E. Krucker-Velasquez and J. W. Swan, Underscreening and hidden ion structures in large scale simulations of concentrated electrolytes, The Journal of Chemical Physics 155, 134903 (2021).

[82] V. Lockett, M. Horne, R. Sedev, T. Rodopoulos, and J. Ralston, Differential capacitance of the double layer at the electrode/ionic liquids interface, Physical Chemistry Chemical Physics 12, 12499 (2010).

[83] D. J. Bozym, B. Uralcan, D. T. Limmer, M. A. Pope, N. J. Szamreta, P. G. Debenedetti, and I. A. Aksay, Anomalous capacitance maximum of the glassy carbon-ionic liquid interface through dilution with organic solvents, The Journal of Physical Chemistry Letters 6, 2644 (2015).

[84] W. J. Hamer and Y.-C. Wu, Osmotic coefficients and mean activity coefficients of uni-univalent electrolytes in water at $25^{\circ} \mathrm{c}$, Journal of Physical and Chemical Reference Data 1, 1047 (1972).

[85] S. W. Coles, C. Park, R. Nikam, M. Kanduč, J. Dzubiella, and B. Rotenberg, Correlation length in concentrated electrolytes: Insights from all-atom molecular dynamics simulations, The Journal of Physical Chemistry B 124, 1778 (2020).

[86] P. Cats, R. Evans, A. Härtel, and R. van Roij, Primitive model electrolytes in the near and far field: Decay lengths from DFT and simulations, The Journal of Chemical Physics 154, 124504 (2021).

[87] N. Anousheh, F. J. Solis, and V. Jadhao, Ionic structure and decay length in highly concentrated confined electrolytes, AIP Advances 10, 125312 (2020).

[88] R. Kjellander, Decay behavior of screened electrostatic surface forces in ionic liquids: the vital role of non-local electrostatics, Physical Chemistry Chemical Physics 18, 18985 (2016).

[89] J. Ulander and R. Kjellander, The decay of pair correlation functions in ionic fluids: a dressed ion theory analysis of Monte Carlo simulations, The Journal of Chemical Physics 114, 4893 (2001).

[90] R. Lhermerout and S. Perkin, Nanoconfined ionic liquids: Disentangling electrostatic and viscous forces, Physical Review Fluids 3, 014201 (2018). 


\title{
Supplementary Material
}

\section{lonic Screening in Bulk and under Confinement}

\author{
Johannes Zeman ${ }^{1}$, Svyatoslav Kondrat ${ }^{2,3,4}$, and Christian Holm*1 \\ ${ }^{1}$ Institute for Computational Physics, University of Stuttgart, D-70569 Stuttgart, Germany \\ ${ }^{2}$ Department of Complex Systems, Institute of Physical Chemistry, Polish Academy of Sciences, \\ Kasprzaka 44/52, 01-224 Warsaw, Poland \\ ${ }^{3}$ Max-Planck-Institut für Intelligente Systeme, Heisenbergstraße 3, 70569 Stuttgart, Germany \\ ${ }^{4}$ IV. Institut für Theoretische Physik, Universität Stuttgart, Pfaffenwaldring 57, 70569 Stuttgart, Germany
}

\section{Contents}

S1 Numerical Methods $\quad$ S2

S1.1 Computation of radial pair distribution functions . . . . . . . . . . . S2

S1.1.1 Distance-dependent statistical accuracy of radial pair distribution functions . . S4

S1.1.2 Error estimation of radial pair distribution functions . . . . . . . . . . . S4

S1.1.3 Error estimation of potentials of mean force . . . . . . . . . . . . S5

$\mathrm{S} 1.2$ Computation of static relative permittivities . . . . . . . . . . . . S6

S1.2.1 Proof of equation S27 (equation 7 in the main article) . . . . . . . S6

S1.2.2 Determining static relative permittivities via the Einstein-Helfand method . . . S8

S1.2.3 Error estimation of static permittivities . . . . . . . . . . . . . S11

S2 Additional Results $\quad$ S13

S2.1 Bulk systems . . . . . . . . . . . . . . . . . . . . . S13

S2.1.1 Volumetric system properties . . . . . . . . . . . . . . . . S13

S2.1.2 Static relative dielectric permittivities . . . . . . . . . . . . . . . . S14

S2.1.3 PMF behavior over time . . . . . . . . . . . . . . . . S15

S2.1.4 Screening length scaling analysis of $\left[\mathrm{C}_{4} \mathrm{C}_{1} \mathrm{Im}\right]^{+}\left[\mathrm{NTf}_{2}\right]^{-}$in propylene carbonate $\mathrm{S} 17$

$\mathrm{S} 2.2$ Confined ionic liquid systems . . . . . . . . . . . . . . . . . . S19

S2.2.1 Number density profiles . . . . . . . . . . . . . . . . . . . . . S19

S2.2.2 Charge density profiles . . . . . . . . . . . . . . . . S20

S2.2.3 Position-dependent RDFs . . . . . . . . . . . . . . . . . . . S21

S2.3 Using reduced-charge ion models in confined systems . . . . . . . . . . . . . . S23

S3 Relating Underscreening to other Measurements $\quad$ S24

S3.1 Differential Capacitance Estimates for Different Widths of the Stern Layer . . . . . . . S24

S4 GROMACS Simulation Parameters $\quad$ S25

$\mathrm{S} 4.1\left[\mathrm{C}_{4} \mathrm{C}_{1} \mathrm{Im}\right]^{+}\left[\mathrm{PF}_{6}\right]^{-}$(all-atom model $) \ldots \ldots \ldots \ldots \ldots \ldots \ldots \ldots \ldots \ldots$

$\mathrm{S} 4.2\left[\mathrm{C}_{4} \mathrm{C}_{1} \mathrm{Im}\right]^{+}\left[\mathrm{PF}_{6}\right]^{-}$(coarse-grained model) $\ldots \ldots \ldots \ldots \ldots \ldots \ldots \ldots$

$\mathrm{S} 4.3$ Aqueous $\mathrm{NaCl}$ solutions . . . . . . . . . . . . . . . . . . . . $\mathrm{S} 27$

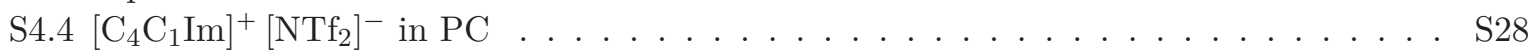

$\mathrm{S} 4.5\left[\mathrm{C}_{4} \mathrm{C}_{1} \mathrm{Im}\right]^{+}\left[\mathrm{NTf}_{2}\right]^{-}$confined between like-charged graphene walls $\ldots \ldots \ldots$. . . . . S S29

$\begin{array}{ll}\text { References } & \text { S30 }\end{array}$

${ }^{*}$ E-mail: holm@icp.uni-stuttgart.de 


\section{S1 Numerical Methods}

\section{S1.1 Computation of radial pair distribution functions}

In homogeneous systems with a fixed number of particles, the radial pair distribution function (RDF) between two molecular species $X$ and $Y$ is formally defined as

$$
g_{X Y}(r)=\frac{\left\langle\rho_{X Y}(r)\right\rangle}{\left\langle\rho_{Y}\right\rangle}
$$

where $\left\langle\rho_{X Y}(r)\right\rangle$ denotes the expected number density of particles of species $Y$ residing on shells of radius $r$ centered at any particle of species $X$, and $\left\langle\rho_{Y}\right\rangle$ is the expected number density of particles of species $Y$ in the entire system, i.e., their bulk number density.

The numerical evaluation of $g_{X Y}(r)$ requires the discretization of space into intervals of width $\Delta r$, so that we define the discretized distance

$$
r_{n}=\left(n+\frac{1}{2}\right) \Delta r, \quad n \in \mathbb{N}_{0} .
$$

In a system with orthorhombic volume $V=L_{x} L_{y} L_{z}$ and periodic boundary conditions in all three dimensions which comprises $N_{X}$ particles of species $X$ and $N_{Y}$ particles of species $Y \neq X$, the RDF is then estimated according to

$$
g_{X Y}\left(r_{n}\right) \approx \hat{g}_{X Y}\left(r_{n}\right):=\frac{\hat{\rho}_{X Y}\left(r_{n}\right)}{\hat{\rho}_{Y}}
$$

with the estimator $\hat{\rho}_{Y}$ for the expected bulk number density of particles of species $Y$

$$
\hat{\rho}_{Y}=\frac{N_{Y}}{\bar{V}},
$$

where we defined the average system volume

$$
\bar{V}:=\frac{1}{N} \sum_{i=1}^{N} V_{i} .
$$

Here, we have averaged over $N$ time steps so that $V_{i}$ denotes the volume of the system at time step $i$, allowing for systems with fluctuating simulation box volumes.

The estimator for the expected number density of particles of species $Y$ residing on shells of radius $r$ centered at any particle of species $X$ is defined as

$$
\hat{\rho}_{X Y}\left(r_{n}\right)=\frac{1}{N N_{X}} \sum_{i=1}^{N} \frac{H_{n, i}^{X Y}}{V_{n, i}^{\text {shell }}},
$$

where $V_{n, i}^{\text {shell }}$ is the shell volume (see below) and the distance histogram $H_{n, i}^{X Y}$ is given as

$$
H_{n, i}^{X Y}:=H_{i}^{X Y}\left(r_{n}\right)=\sum_{j=1}^{N_{X}} \sum_{k=1}^{N_{Y}} \delta\left(\left\lfloor\frac{\left\|\boldsymbol{r}_{k, i}-\boldsymbol{r}_{j, i}\right\|_{\mathrm{pbc}}}{\Delta r}\right\rfloor-n\right) .
$$

Again, we averaged over configurations of $N$ time steps so that $\boldsymbol{r}_{j, i}$ and $\boldsymbol{r}_{k, i}$ are the positions of the particles $j$ and $k$ at time step $i$. The function $\delta(\cdot)$ is the discrete Dirac delta function, which is defined as

$$
\delta(x)= \begin{cases}1 & \text { if } x=0 \\ 0 & \text { otherwise }\end{cases}
$$


and the floor operator $\lfloor\cdot\rfloor$ yields the closest integer smaller than or equal to its argument. The operator $\|\cdot\|_{\mathrm{pbc}}$ is the Euclidean $L_{2}$-norm (i.e., distance) with periodic boundary conditions applied according to the minimum image convention

$$
\|\boldsymbol{r}\|_{\mathrm{pbc}}^{2}=\sum_{d \in\{x, y, z\}}\left(\left(r_{d} \bmod L_{d}\right)+h\left(-\left(r_{d} \bmod L_{d}\right)-\frac{L_{d}}{2}\right) L_{d}-h\left(\left(r_{d} \bmod L_{d}\right)-\frac{L_{d}}{2}\right) L_{d}\right)^{2}
$$

where $h(\cdot)$ denotes the Heaviside step function defined as

$$
h(x)= \begin{cases}1 & \text { if } x>0 \\ 0 & \text { otherwise }\end{cases}
$$

The shell volumes $V_{n, i}^{\text {shell }}:=V_{i}^{\text {shell }}\left(r_{n}\right)$ could in principle be calculated from the volume difference of two spheres with radii

$$
\begin{aligned}
& R_{n}^{\text {outer }}=r_{n}+\frac{\Delta r}{2}=(n+1) \Delta r \\
& R_{n}^{\text {inner }}=r_{n}-\frac{\Delta r}{2}=n \Delta r .
\end{aligned}
$$

Although seemingly trivial, this requires particular attention. According to the minimum image convention defined in eq. (S9), the maximum possible distance in a 3d-periodic orthorhombic box with edge lengths $L_{x, i}, L_{y, i}, L_{z, i}$ at time step $i$ is

$$
r_{i}^{\max }:=\left\|\boldsymbol{r}_{i}^{\max }\right\|_{\mathrm{pbc}}=\frac{1}{2} \sqrt{L_{x, i}^{2}+L_{y, i}^{2}+L_{z, i}^{2}} .
$$

However, the radius of the largest sphere that is completely enclosed by the box at all time steps $i$ is

$$
R_{\mathrm{enc}}^{\max }=\frac{1}{2} \min _{i}\left(L_{x, i}, L_{y, i}, L_{z, i}\right) .
$$

Thus, if and only if we limit the evaluation of $\hat{g}_{X Y}\left(r_{n}\right)$ to distances $r_{n} \leqslant R_{\mathrm{enc}}^{\max }-\frac{\Delta r}{2}$, the shell volumes are time-invariant and can be obtained from

$$
V_{n, i}^{\text {shell }} \equiv V_{n}^{\text {shell }}=\frac{4 \pi}{3}\left(((n+1) \Delta r)^{3}-(n \Delta r)^{3}\right), \quad n \leqslant \frac{R_{\mathrm{enc}}^{\max }}{\Delta r}+1 .
$$

Finally, the complete equation for the numerical RDF estimator reads

$$
\hat{g}_{X Y}\left(r_{n}\right)=\left(\frac{1}{N N_{Y}} \sum_{i=1}^{N} V_{i}\right) \frac{1}{N N_{X} V_{n}^{\text {shell }}} \sum_{i=1}^{N} \sum_{j=1}^{N_{X}} \sum_{k=1}^{N_{Y}} \delta\left(\left\lfloor\frac{\left\|\boldsymbol{r}_{k, i}-\boldsymbol{r}_{j, i}\right\|_{\mathrm{pbc}}}{\Delta r}\right\rfloor-n\right) .
$$

If the RDF is to be evaluated among particles of the same species $X$, we want to exclude pair distances of particles paired with themselves, which is achieved by removing the pairs $(j, k)$ with $j=k$ from the histogram summation. The corresponding RDF estimator then becomes

$$
\hat{g}_{X X}\left(r_{n}\right)=\left(\frac{1}{N N_{X}} \sum_{i=1}^{N} V_{i}\right) \frac{2}{N N_{X} V_{n}^{\text {shell }}} \sum_{i=1}^{N} \sum_{j=1}^{N_{X}} \sum_{k=j+1}^{N_{X}} \delta\left(\left\lfloor\frac{\left\|\boldsymbol{r}_{k, i}-\boldsymbol{r}_{j, i}\right\|_{\mathrm{pbc}}}{\Delta r}\right\rfloor-n\right),
$$

where we additionally exploited the symmetry of the Euclidean distance by shifting the initial index of the innermost sum and multiplying by 2 . 


\section{S1.1.1 Distance-dependent statistical accuracy of radial pair distribution functions}

Let us consider an ideal gas in a 3d-periodic orthorhombic volume with constant edge lengths $L_{x}, L_{y}, L_{z}$ comprising a fixed number of particles $N_{X}$ at thermal equilibrium. Then, by definition of an ideal gas, the expected number density in any part of the system equals the bulk density so that the expected value of $g_{X X}(r)$ is $\left\langle g_{X X}(r)\right\rangle=1$ for all possible distances $r$. However, if we measure the estimator $\hat{g}_{X X}\left(r_{n}\right)$ of this system for a given number of time steps $N$, this measurement will be subject to a statistical error $\epsilon\left(\hat{g}_{X X}\left(r_{n}\right)\right)$. Because of the constant volume and fixed number of particles, the measured bulk number density $\hat{\rho}_{X}$ will be time-invariant and equal to its expected value $\left\langle\rho_{X}\right\rangle=\frac{N_{X}}{V}$. Thus, the statistical error can only be due to the estimator $\hat{\rho}_{X X}\left(r_{n}\right)$. If we assume that the time $\Delta t$ between measurements is large enough for the system to decorrelate, the different measurements of $\hat{\rho}_{X X}\left(r_{n}\right)$ at subsequent time steps will be uncorrelated. The statistical error $\epsilon\left(\hat{\rho}_{X X}\left(r_{n}\right)\right)$ should then be proportional to $\frac{1}{\sqrt{N}}$ for all $r_{n}$. Nevertheless, the expected value of this error will also depend on the square root of the expected number of particle distances $\left\langle N^{\text {dist }}\left(r_{n}\right)\right\rangle$ contributing to the histogram $H_{n}^{X X}$. According to eq. (S15),

$$
\left\langle N^{\text {dist }}\left(r_{n}\right)\right\rangle=\frac{1}{2} N_{X}\left(N_{X}-1\right) \frac{V_{n}^{\text {shell }}}{V} .
$$

Since both $N_{X}$ and $V$ are constant, we conjecture that

$$
\left\langle\epsilon\left(\hat{g}_{X X}\left(r_{n}\right)\right)\right\rangle \propto \frac{1}{\sqrt{V_{n}^{\text {shell }}}} .
$$

\section{S1.1.2 Error estimation of radial pair distribution functions}

Whenever $g_{X Y}(r)$ is estimated in systems with constant simulation box volumes $V_{i}=\bar{V}=V$, then $\left\langle\rho_{Y}\right\rangle=\rho_{Y}=\frac{N_{Y}}{V}$ at all time steps. It follows that

$$
\left\langle g_{X Y}(r)\right\rangle=\frac{\left\langle\rho_{X Y}(r)\right\rangle}{\left\langle\rho_{Y}\right\rangle}=\frac{V}{N_{Y}}\left\langle g_{X Y}(r)\right\rangle=\left\langle\frac{\rho_{X Y}(r)}{\rho_{Y}}\right\rangle \quad \text { if } V=\text { const. }
$$

For the estimator $\hat{g}_{X Y}\left(r_{n}\right)$, it follows analogously that it can be expressed as the time average

$$
\hat{g}_{X Y}\left(r_{n}\right)=\frac{1}{N} \sum_{i=1}^{N} \hat{g}_{X Y, i}\left(r_{n}\right) \text { if } V=\text { const },
$$

where $\hat{g}_{X Y, i}\left(r_{n}\right)$ denotes $\hat{g}_{X Y}\left(r_{n}\right)$ evaluated at a single time step $i$. In other words, $\hat{g}_{X Y}\left(r_{n}\right)$ is the mean of the time series $\hat{g}_{X Y}\left(r_{n}\right)(t)$. Thus, we can estimate the error $\epsilon\left(\hat{g}_{X Y}\left(r_{n}\right)\right)$ separately for each distance $r_{n}$. Since we cannot safely assume that subsequently measured values $\hat{g}_{X Y, i}\left(r_{n}\right), \hat{g}_{X Y, i+j}\left(r_{n}\right)$ are uncorrelated, we have to compute the error estimate according to

$$
\hat{\epsilon}\left(\hat{g}_{X Y}\left(r_{n}\right)\right)=\sqrt{\frac{1}{N} \sum_{j=-j_{\max }}^{j_{\max }} b \hat{R}_{j}^{\hat{g} \hat{g}}\left(r_{n}\right)}
$$

with the biased (hence the prescript $b$ ) auto-covariance estimator ${ }^{b} \hat{R}_{j}^{\hat{g} \hat{g}}\left(r_{n}\right)$, which reads

$$
{ }^{b} \hat{R}_{j}^{\hat{g} \hat{g}}\left(r_{n}\right)=\frac{1}{N} \sum_{i=1}^{N-|j|}\left(\hat{g}_{X Y, i}\left(r_{n}\right)-\frac{1}{N} \sum_{k=1}^{N} \hat{g}_{X Y, k}\left(r_{n}\right)\right)\left(\hat{g}_{X Y, i+j}\left(r_{n}\right)-\frac{1}{N} \sum_{k=1}^{N} \hat{g}_{X Y, k}\left(r_{n}\right)\right) .
$$

Of course, due to the the convolution theorem, ${ }^{b} \hat{R}_{j}^{\hat{g} \hat{g}}\left(r_{n}\right)$ can be equivalently evaluated using Fourier transforms for reasons of computational efficiency. 
For systems with fluctuating box volumes, eq. (S18) does not strictly hold because generally, $V_{i} \neq \bar{V}$. Nevertheless, we can follow the same procedure as described above because $\left\langle g_{X Y}(r)\right\rangle \approx\left\langle\frac{\rho_{X Y}(r)}{\rho_{Y}}\right\rangle$ is a sufficiently good approximation for large $N_{X}, N_{Y}$, and $N$ by virtue of the law of large numbers.

It is important to note, however, that there is absolutely no justification for using this approximation for any other purpose except for estimating $\hat{\epsilon}\left(\hat{g}_{X Y}\left(r_{n}\right)\right)$. In particular, under no circumstances should it be used for evaluating $\hat{g}_{X Y}\left(r_{n}\right)$ because neither could this improve computational efficiency nor numerical accuracy. After all, if anything, it would yield biased results!

\section{S1.1.3 Error estimation of potentials of mean force}

The PMF $w_{X Y}(r)$ between species $X$ and $Y$ is computed from the natural logarithm of the corresponding RDF $g_{X Y}(r)$ according to

$$
w_{X Y}(r)=-k_{\mathrm{B}} T \ln \left(g_{X Y}(r)\right) .
$$

For large $r, g_{X Y}(r) \approx 1$, so that we can approximate the logarithm by its first-order Taylor expansion around 1 , which is $\left.\ln (x)\right|_{1}=x-1+\mathcal{O}\left((x-1)^{2}\right)$. This linearization allows us to approximate $w_{X Y}(r)$ as

$$
w_{X Y}(r) \approx k_{\mathrm{B}} T\left(\frac{\left\langle\rho_{X Y}(r)\right\rangle}{\left\langle\rho_{Y}\right\rangle}-1\right) \text { for large } r
$$

so that by using again the approximation $\left\langle g_{X Y}(r)\right\rangle \approx\left\langle\frac{\rho_{X Y}(r)}{\rho_{Y}}\right\rangle$, we can obtain the statistical error of the estimator $\hat{w}_{X Y}(r)$ in the same manner as we have done for $\hat{g}_{X Y}(r)$, reading

$$
\hat{\epsilon}\left(\hat{w}_{X Y}\left(r_{n}\right)\right)=\sqrt{\frac{1}{N} \sum_{j=-N}^{N} b \hat{R}_{j}^{\hat{w} \hat{w}}\left(r_{n}\right)}
$$

with

$$
{ }^{b} \hat{R}_{j}^{\hat{w} \hat{w}}\left(r_{n}\right)=\frac{1}{N} \sum_{i=1}^{N-|j|}\left(\hat{w}_{X Y, i}\left(r_{n}\right)-\frac{1}{N} \sum_{k=1}^{N} \hat{w}_{X Y, k}\left(r_{n}\right)\right)\left(\hat{w}_{X Y, i+j}\left(r_{n}\right)-\frac{1}{N} \sum_{k=1}^{N} \hat{w}_{X Y, k}\left(r_{n}\right)\right) .
$$

Since the linearization is only valid for large $r$ where $g_{X Y}(r) \approx 1$, this error estimate is not exact for $r \rightarrow 0$. Nevertheless, since the statistical error analysis is only relevant for the PMF's asymptotic behavior at large separations, this approach is sufficient for our purposes. 


\section{S1.2 Computation of static relative permittivities}

In Molecular Dynamics (MD) simulations employing periodic boundary conditions (PBC) in all three dimensions and electrostatic tinfoil boundary conditions at infinity, the simulated system's static relative permittivity $\varepsilon_{r}$ can be computed according to ${ }^{\mathrm{S} 1}$

$$
\varepsilon_{r}=1+\frac{\left\langle\boldsymbol{M}^{2}\right\rangle}{3 V k_{\mathrm{B}} T \varepsilon_{0}},
$$

where $V$ is the simulation box volume, $k_{\mathrm{B}}$ the Boltzmann constant, $T$ the system's absolute temperature, the operator $\langle\cdot\rangle$ returns its argument's expected value, and $M$ denotes the system's itinerant total dipole moment. In systems with free ionic charges, the latter is ill-defined ${ }^{\mathrm{S} 2}$ and cannot be measured directly. Instead, $\left\langle\boldsymbol{M}^{2}\right\rangle$ can be obtained from the itinerant total current $\boldsymbol{J}$ according to

$$
\left\langle\boldsymbol{M}^{2}\right\rangle=-\lim _{t \rightarrow \infty} \int_{0}^{t} \tau\langle\boldsymbol{J}(0) \boldsymbol{J}(\tau)\rangle \mathrm{d} \tau,
$$

where $\boldsymbol{J}(t)$ is defined as

$$
\boldsymbol{J}(t)=\frac{\mathrm{d}}{\mathrm{d} t} \boldsymbol{M}(t)
$$

and computed from

$$
\boldsymbol{J}(t)=\sum_{i} q_{i} \boldsymbol{v}_{i}(t)
$$

where the sum runs over all atoms $i$ in the system with charges $q_{i}$ and velocities $\boldsymbol{v}_{i}$. Since all $q_{i}$ are constant, and the velocities $\boldsymbol{v}_{i}(t)$ are independent of the origin of the spatial coordinate system and remain continuous in time even when ions move across periodic boundaries, $\boldsymbol{J}(t)$ is a well-defined and directly measurable quantity.

\section{S1.2.1 Proof of equation S27 (equation 7 in the main article)}

We recall the definition of the autocorrelation function of a continuous time-dependent variable $A(t) \in \mathbb{R}$

$$
R^{A A}(\tau):=\langle A(t) A(t+\tau)\rangle
$$

where the symmetry

$$
R^{A A}(\tau)=\langle A(t) A(t+\tau)\rangle \stackrel{t \rightarrow t-\tau}{=}\langle A(t-\tau) A(t)\rangle=R^{A A}(-\tau)
$$

holds. Provided that $A(t)$ is differentiable in $t$, by applying the chain rule, we see that its derivative $\dot{R}^{A A}(\tau):=\frac{\mathrm{d}}{\mathrm{d} \tau} R^{A A}(\tau)$ is

$$
\dot{R}^{A A}(\tau)=\langle A(t) \dot{A}(t+\tau)\rangle=R^{A \dot{A}}(\tau)
$$

and

$$
\dot{R}^{A A}(\tau)=-\langle\dot{A}(t-\tau) A(t)\rangle \stackrel{t \rightarrow t+\tau}{=}-\langle\dot{A}(t) A(t+\tau)\rangle=-R^{\dot{A} A}(\tau) .
$$

For the second derivative $\ddot{R}^{A A}(\tau):=\frac{\mathrm{d}^{2}}{\mathrm{~d} t^{2}} R^{A A}(\tau)$, it follows analogously that

$$
\ddot{R}^{A A}(\tau)=-R^{\dot{A} \dot{A}}(\tau)
$$

Likewise, the indefinite integral of $R^{\dot{A} \dot{A}}(\tau)$ is

$$
\begin{aligned}
\int R^{\dot{A} \dot{A}}(\tau) \mathrm{d} \tau & =\left\langle\dot{A}(t) \int \dot{A}(t+\tau) \mathrm{d} \tau\right\rangle=\langle\dot{A}(t)(A(t+\tau)+C)\rangle \\
& =R^{\dot{A} A}(\tau)+C\langle\dot{A}(t)\rangle=-R^{A \dot{A}}(\tau)+C\langle\dot{A}(t)\rangle
\end{aligned}
$$

with the constant of integration $C$. 
Both the total dipole moment $\boldsymbol{M}$ and the total current $\boldsymbol{J}$ in a finite volume of a system in nonsolid state at thermodynamic equilibrium are real-valued, continuously differentiable, and weakly stationary random variables with zero mean. Weak stationarity means that their distributions and, thus, their expected values are time-invariant, so that their autocorrelation functions can be expressed as

$$
\begin{aligned}
R^{M M}(\tau) & =\langle\boldsymbol{M}(t) \boldsymbol{M}(t+\tau)\rangle=\langle\boldsymbol{M}(0) \boldsymbol{M}(\tau)\rangle, \\
R^{J J}(\tau) & =\langle\boldsymbol{J}(t) \boldsymbol{J}(t+\tau)\rangle=\langle\boldsymbol{J}(0) \boldsymbol{J}(\tau)\rangle .
\end{aligned}
$$

Since $\boldsymbol{M}(t)$ is continuously differentiable, we can express its value at time $t$ as

$$
\boldsymbol{M}(t)=\boldsymbol{M}\left(t_{0}\right)+\int_{t_{0}}^{t}\left(\frac{\mathrm{d}}{\mathrm{d} \tau} \boldsymbol{M}(\tau)\right) \mathrm{d} \tau .
$$

By inserting the definition (S28) and setting $t_{0}=0$, we obtain

$$
\int_{0}^{t} \boldsymbol{J}(\tau) \mathrm{d} \tau=\boldsymbol{M}(t)-\boldsymbol{M}(0) .
$$

Integrating the RHS of eq. (S27) by parts yields

$$
\begin{aligned}
-\lim _{t \rightarrow \infty} \int_{0}^{t} \tau\langle\boldsymbol{J}(0) \boldsymbol{J}(\tau)\rangle \mathrm{d} \tau=-\lim _{t \rightarrow \infty} & \left(\left[\tau \int\langle\boldsymbol{J}(0) \boldsymbol{J}(\tau)\rangle \mathrm{d} \tau\right]_{0}^{t}\right. \\
& \left.-\int_{0}^{t}\left(\int\langle\boldsymbol{J}(0) \boldsymbol{J}(\tau)\rangle \mathrm{d} \tau\right) \mathrm{d} \tau\right) .
\end{aligned}
$$

By exploiting the properties of the integrals of autocorrelation functions according to eq. (S35), we obtain

$$
\begin{aligned}
-\lim _{t \rightarrow \infty} \int_{0}^{t} \tau\langle\boldsymbol{J}(0) \boldsymbol{J}(\tau)\rangle \mathrm{d} \tau & =\lim _{t \rightarrow \infty}\left([\tau\langle\boldsymbol{M}(0) \boldsymbol{J}(\tau)\rangle+\tau C \underbrace{\langle\boldsymbol{J}(\tau)\rangle}_{=0}]_{0}^{t}-\int_{0}^{t}(\langle\boldsymbol{M}(0) \boldsymbol{J}(\tau)\rangle+C \underbrace{\langle\boldsymbol{J}(\tau)\rangle}_{=0}) \mathrm{d} \tau\right) \\
& =\lim _{t \rightarrow \infty}(\underbrace{0\langle\boldsymbol{M}(0) \boldsymbol{J}(0)\rangle}_{=0}+t\langle\boldsymbol{M}(0) \boldsymbol{J}(t)\rangle-\int_{0}^{t}\langle\boldsymbol{M}(0) \boldsymbol{J}(\tau)\rangle \mathrm{d} \tau) \\
& =\underbrace{\lim _{t \rightarrow \infty}(t\langle\boldsymbol{M}(0) \boldsymbol{J}(t)\rangle)}_{=0}-\lim _{t \rightarrow \infty}\left\langle\boldsymbol{M}(0) \int_{0}^{t} \boldsymbol{J}(\tau) \mathrm{d} \tau\right\rangle
\end{aligned}
$$

The first term of the RHS of eq. (S39) vanishes because asymptotically, $\langle\boldsymbol{M}(0) \boldsymbol{J}(t)\rangle$ decays exponentially with $t$.

Inserting eq. (S38) into eq. (S39) yields

$$
\begin{aligned}
-\lim _{t \rightarrow \infty} \int_{0}^{t} \tau\langle\boldsymbol{J}(0) \boldsymbol{J}(\tau)\rangle \mathrm{d} \tau & =-\lim _{t \rightarrow \infty}\langle\boldsymbol{M}(0)[\boldsymbol{M}(t)-\boldsymbol{M}(0)]\rangle . \\
& =-\underbrace{\lim _{t \rightarrow \infty}\langle\boldsymbol{M}(0) \boldsymbol{M}(t)\rangle}_{=0}+\lim _{t \rightarrow \infty}\langle\boldsymbol{M}(0) \boldsymbol{M}(0)\rangle \\
& =\left\langle\boldsymbol{M}^{2}\right\rangle,
\end{aligned}
$$

proving the validity of eq. (S27) 


\section{S1.2.2 Determining static relative permittivities via the Einstein-Helfand method}

In practice, the tail of the current autocorrelation function $\langle\boldsymbol{J}(0) \boldsymbol{J}(\tau)\rangle$ suffers from statistical noise, which is amplified by the factor $\tau$ in the integral of eq. (S27). Thus, determining $\left\langle\boldsymbol{M}^{2}\right\rangle$ directly from eq. (S27) is infeasible. This issue is clearly visible in fig. S1, which shows the autocorrelation $\langle\boldsymbol{J}(0) \boldsymbol{J}(\tau)\rangle$ (panel a), its running integral $\int_{0}^{\tau}\left\langle\boldsymbol{J}(0) \boldsymbol{J}\left(\tau^{\prime}\right)\right\rangle \mathrm{d} \tau^{\prime}$ (panel $\mathbf{b}$ ), and the negative integral of the lag timeweighted autocorrelation $-\int_{0}^{\tau} \tau^{\prime}\left\langle\boldsymbol{J}(0) \boldsymbol{J}\left(\tau^{\prime}\right)\right\rangle \mathrm{d} \tau^{\prime}$ (panel c) of the current $\boldsymbol{J}$ obtained from simulations of a $50 \mathrm{~mol} \%$ solution of $\left[\mathrm{C}_{4} \mathrm{C}_{1} \mathrm{Im}\right]^{+}\left[\mathrm{NTf}_{2}\right]^{-}$in propylene carbonate.

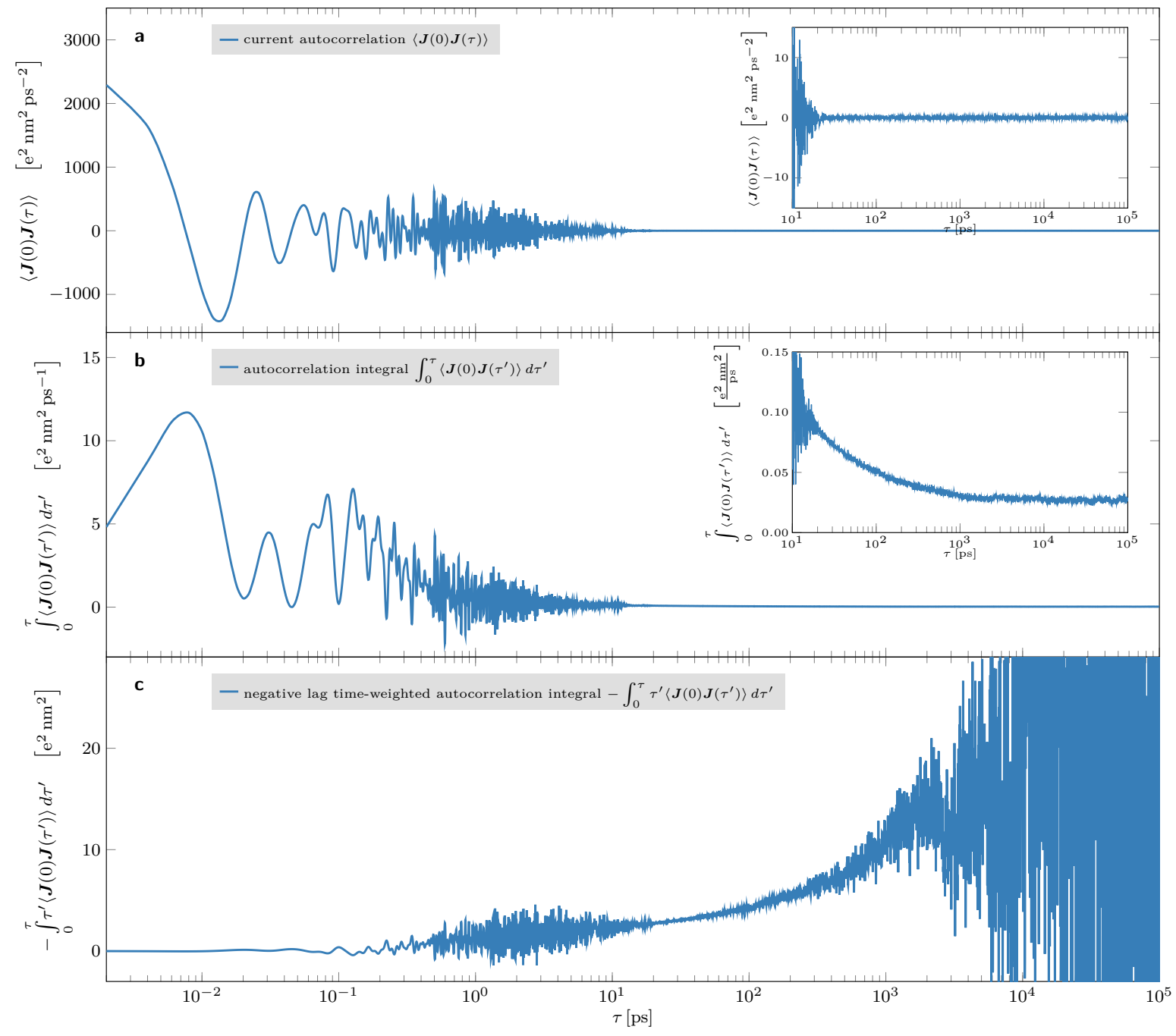

Figure S1: Current autocorrelation data obtained from simulations of a solution of $\left[\mathrm{C}_{4} \mathrm{C}_{1} \mathrm{Im}\right]^{+}\left[\mathrm{NTf}_{2}\right]^{-}$in propylene carbonate at $T=$ $300 \mathrm{~K}$ with an IL mole fraction of $x_{\mathrm{IL}}=0.5$ (concentration $c_{\mathrm{IL}}=2.75 \mathrm{~mol} / \mathrm{I}$ ) comprising 500 ion pairs and 500 solvent molecules. The current $\boldsymbol{J}(t)$ was computed according to eq. (S29) and recorded at every time step ( $\Delta t=2 \mathrm{fs}$ ) during the simulation time of $t_{\mathrm{sim}} \approx 1074 \mathrm{~ns}\left(2^{29}\right.$ time steps) per run from three independent simulation runs. For each run, the autocorrelation functions $\left\langle J_{d}(0) J_{d}(\tau)\right\rangle$ were computed for each spatial dimension $d \in\{x, y, z\}$, summed up over $d$ to obtain $\langle\boldsymbol{J}(0) \boldsymbol{J}(\tau)\rangle$, and finally averaged over all runs.

Panel a: Current autocorrelation $\langle\boldsymbol{J}(0) \boldsymbol{J}(\tau)\rangle$. Inset: Magnification of the tail region $\left(10 \leqslant \tau \leqslant 10^{5} \mathrm{ps}\right)$. The autocorrelation seems to have converged to zero at $\tau \approx 30 \mathrm{ps}$, and the remaining tail appears to be random noise.

Panel b: Running integral $\int_{0}^{\tau}\left\langle\boldsymbol{J}(0) \boldsymbol{J}\left(\tau^{\prime}\right)\right\rangle \mathrm{d} \tau^{\prime}$ of the current autocorrelation. Inset: Magnification of the tail region $\left(10 \leqslant \tau \leqslant 10^{5} \mathrm{ps}\right)$, from which it becomes evident that the seemingly random noise in the autocorrelation's tail actually still contains information up to $\tau \approx 3 \cdot 10^{3} \mathrm{ps}$, where the integral has converged to a value of about $0.027 \mathrm{e}^{2} \mathrm{~nm}^{2} \mathrm{ps}^{-1}$.

Panel c: Negative running integral $\int_{0}^{\tau} \tau^{\prime}\left\langle\boldsymbol{J}(0) \boldsymbol{J}\left(\tau^{\prime}\right)\right\rangle \mathrm{d} \tau^{\prime}$ of the lag time-weighted current autocorrelation (RHS of eq. (S27)). Due to the multiplication with the lag time, the noise in the current autocorrelation's tail is amplified, which makes it practically impossible to obtain a meaningful estimate of the integral's asymptotic value $\left\langle\boldsymbol{M}^{2}\right\rangle$. 
An alternative approach is to estimate $\left\langle\boldsymbol{M}^{2}\right\rangle$ from the itinerant dipole moment's mean square displacement $\left\langle(\Delta \boldsymbol{M})^{2}(t)\right\rangle=\left\langle[\boldsymbol{M}(t)-\boldsymbol{M}(0)]^{2}\right\rangle$, which will be discussed in the following.

According to eq. (S38), we can express $\left\langle(\Delta \boldsymbol{M})^{2}(t)\right\rangle$ as

$$
\begin{aligned}
\left\langle(\Delta \boldsymbol{M})^{2}(t)\right\rangle & =\left\langle\left(\int_{0}^{t} \boldsymbol{J}\left(t^{\prime}\right) \mathrm{d} t^{\prime}\right)^{2}\right\rangle \\
& =\int_{0}^{t}\left(\int_{0}^{t}\left\langle\boldsymbol{J}\left(t^{\prime}\right) \boldsymbol{J}\left(t^{\prime \prime}\right)\right\rangle \mathrm{d} t^{\prime \prime}\right) \mathrm{d} t^{\prime},
\end{aligned}
$$

where we identify $\left\langle\boldsymbol{J}\left(t^{\prime}\right) \boldsymbol{J}\left(t^{\prime \prime}\right)\right\rangle$ as the autocorrelation function of the itinerant current $\boldsymbol{J}$ centered at $t^{\prime}$. Since $\left\langle\boldsymbol{J}\left(t^{\prime}\right) \boldsymbol{J}\left(t^{\prime \prime}\right)\right\rangle$ is symmetric around $t^{\prime}$, we can rewrite eq. (S41) as

$$
\left\langle(\Delta \boldsymbol{M})^{2}(t)\right\rangle=2 \int_{0}^{t}\left(\int_{0}^{t^{\prime}}\left\langle\boldsymbol{J}\left(t^{\prime}\right) \boldsymbol{J}\left(t^{\prime \prime}\right)\right\rangle \mathrm{d} t^{\prime \prime}\right) \mathrm{d} t^{\prime} .
$$

Furthermore, at equilibrium, the autocorrelation function is invariant under translations in time, so that translating by $-t^{\prime}$ and substituting $t^{\prime \prime}-t^{\prime} \rightarrow \tau$ yields

$$
\left\langle(\Delta \boldsymbol{M})^{2}(t)\right\rangle=2 \int_{0}^{t}\left(\int_{0}^{t^{\prime}}\langle\boldsymbol{J}(0) \boldsymbol{J}(\tau)\rangle \mathrm{d} \tau\right) \mathrm{d} t^{\prime} .
$$

We can now change the order of integration by expressing the ranges of integration as

$$
\underbrace{\left(0 \leqslant \tau \leqslant t^{\prime}\right)}_{\text {inner }} \wedge \underbrace{\left(0 \leqslant t^{\prime} \leqslant t\right)}_{\text {outer }} \Leftrightarrow\left(0 \leqslant \tau \leqslant t^{\prime} \leqslant t\right) \Leftrightarrow \underbrace{\left(\tau \leqslant t^{\prime} \leqslant t\right)}_{\text {inner }} \wedge \underbrace{(0 \leqslant \tau \leqslant t)}_{\text {outer }}
$$

to obtain

$$
\begin{aligned}
\left\langle(\Delta \boldsymbol{M})^{2}(t)\right\rangle & =2 \int_{0}^{t}\left(\int_{\tau}^{t}\langle\boldsymbol{J}(0) \boldsymbol{J}(\tau)\rangle \mathrm{d} t^{\prime}\right) \mathrm{d} \tau \\
& =2 \int_{0}^{t}\langle\boldsymbol{J}(0) \boldsymbol{J}(\tau)\rangle\left(\int_{\tau}^{t} \mathrm{~d} t^{\prime}\right) \mathrm{d} \tau \\
& =2 \int_{0}^{t}\langle\boldsymbol{J}(0) \boldsymbol{J}(\tau)\rangle(t-\tau) \mathrm{d} \tau \\
& =2 t \int_{0}^{t}\langle\boldsymbol{J}(0) \boldsymbol{J}(\tau)\rangle \mathrm{d} \tau-2 \int_{0}^{t} \tau\langle\boldsymbol{J}(0) \boldsymbol{J}(\tau)\rangle \mathrm{d} \tau .
\end{aligned}
$$

In the long time limit $t \gg \tau_{\mathrm{c}}$ far beyond the autocorrelation time $\tau_{\mathrm{c}}$, both integrals converge to a constant so that

$$
\begin{aligned}
\lim _{t \gg \tau_{c}}\left(\left\langle(\Delta \boldsymbol{M})^{2}(t)\right\rangle\right) & =2 t \lim _{t \gg \tau_{c}}\left(\int_{0}^{t}\langle\boldsymbol{J}(0) \boldsymbol{J}(\tau)\rangle \mathrm{d} \tau\right)-2 \lim _{t \gg \tau_{c}}\left(\int_{0}^{t} \tau\langle\boldsymbol{J}(0) \boldsymbol{J}(\tau)\rangle \mathrm{d} \tau\right) \\
: & =c_{1} t+c_{2}
\end{aligned}
$$


approaches a linear time dependence for large $t$ with slope $c_{1}$ and offset $c_{2}$. A comparison with eq. (S27) immediately shows that $c_{2}=2\left\langle\boldsymbol{M}^{2}\right\rangle$. Thus, by fitting a linear function $f(t)=c_{1} t+c_{2}$ to $\left\langle(\Delta \boldsymbol{M})^{2}(t)\right\rangle$ for large $t$, we can obtain the relative permittivity of the system according to

$$
\varepsilon_{r}=1+\frac{c_{2}}{6 V k_{\mathrm{B}} T \varepsilon_{0}} .
$$

It is important to note that the onset of the linear regime, and, therefore, the start of the fitting range, is generally difficult to determine from $\left\langle(\Delta \boldsymbol{M})^{2}(t)\right\rangle$. Instead, it should be chosen as the time where the integrated current autocorrelation $\int_{0}^{t}\langle\boldsymbol{J}(0) \boldsymbol{J}(\tau)\rangle \mathrm{d} \tau$ has converged to a constant because this is much easier to identify.

Figure S2 shows the itinerant dipole moment's mean square displacement $\left\langle(\Delta \boldsymbol{M})^{2}(t)\right\rangle$ computed according to eq. (S45) from the autocorrelation data displayed in fig. S1a. The curve looks intriguingly

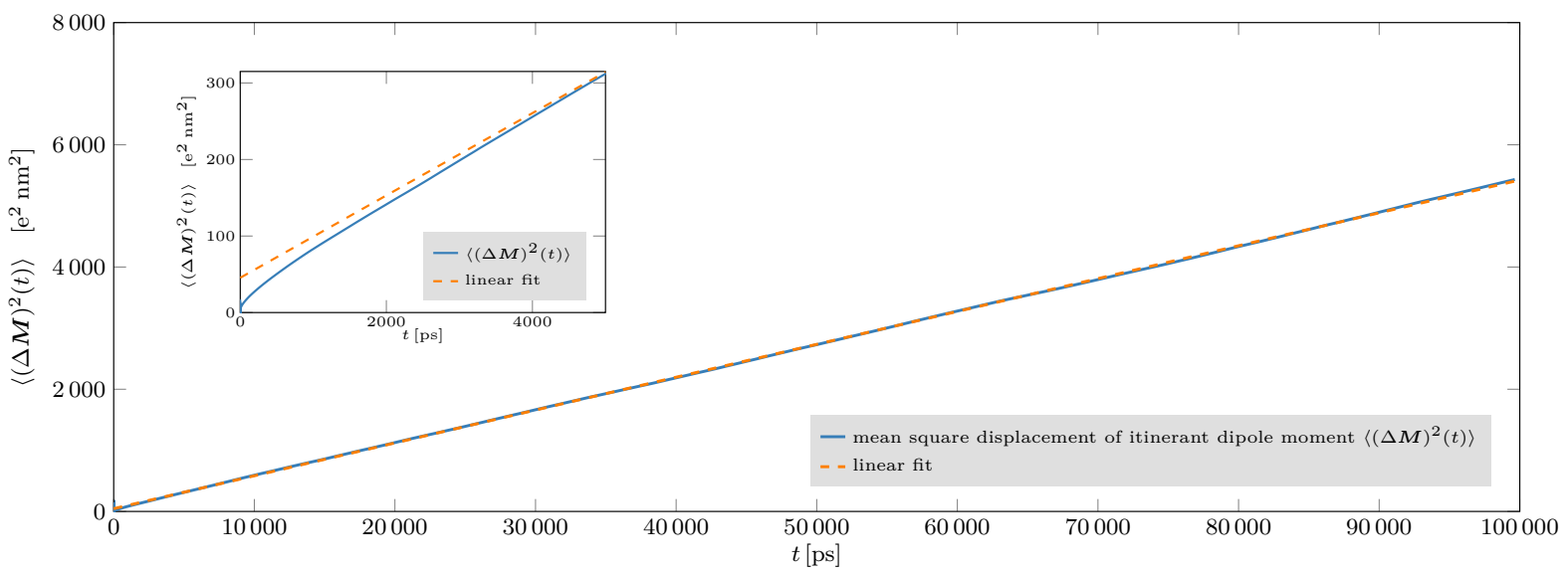

Figure S2: Mean square displacement $\left\langle(\Delta M)^{2}(t)\right\rangle$ of the system's itinerant dipole moment $\boldsymbol{M}$ (solid blue line) computed according to eq. (S45) from the autocorrelation data displayed in fig. S1a. The dashed orange line represents a least-squares fit of the linear function $f(t)=c_{1} t+c_{2}$ (eq. (S46)) in the range $3 \cdot 10^{3} \leqslant t \leqslant 10^{5}$, which appears to fit the data extremely well. As discussed in the text, this seemingly perfect fit is misleading, and the actual error is much larger than what one would infer from the covariance matrix of the least squares fit. Inset: Magnification of the mean square displacement's short time behavior (blue line). According to eqs. (S27) and (S46), the y-axis offset $c_{2}$ can be used to estimate $\left\langle\boldsymbol{M}^{2}\right\rangle=\frac{1}{2} c_{2}$.

linear, so that the least-squares fit (dashed orange line) to the linear function $f(t)=c_{1} t+c_{2}$ (eq. (S46)) in the range $3 \cdot 10^{3} \leqslant t \leqslant 10^{5}$ matches the data almost perfectly. The fit parameters and corresponding error estimates $c_{1}=0.05378679 \pm 5.9 \cdot 10^{-8} \mathrm{e}^{2} \mathrm{~nm}^{2} \mathrm{ps}^{-1}$ and $c_{2}=45.4505 \pm 3.4 \cdot 10^{-3} \mathrm{e}^{2} \mathrm{~nm}^{2}$ confirm this. If we now compute the system's static relative permittivity according to eq. (S47), we obtain

$$
\varepsilon_{r}=1+\frac{(45.4505 \pm 0.0034) \mathrm{e}^{2} \mathrm{~nm}^{2}}{6 \cdot(301.7629 \pm 0.0026) \mathrm{nm}^{3} \cdot \varepsilon_{0} \cdot k_{\mathrm{B}} \cdot 300 \mathrm{~K}}=18.5707 \pm 0.0013 .
$$

While the value itself seems reasonable, the error estimate is suspiciously small. In fact, this is for two reasons: (i) the time series used to compute $\left\langle(\Delta \boldsymbol{M})^{2}(t)\right\rangle$ are strongly correlated, which means that the data entering the fit is heavily oversampled, and (ii) the statistical error of $\left\langle(\Delta \boldsymbol{M})^{2}(t)\right\rangle$ is not taken into account. As we will see in the following, even though $\left\langle(\Delta \boldsymbol{M})^{2}(t)\right\rangle$ appears to be perfectly linear in the long time limit, its statistical error is in fact quite substantial. 


\section{S1.2.3 Error estimation of static permittivities}

As mentioned earlier, the analysis of the itinerant dipole moment's mean square displacement $\left\langle(\Delta \boldsymbol{M})^{2}(t)\right\rangle$ in the previous section was based on current autocorrelation data obtained from three independent simulation runs, so that one can perform separate analyses for each system to obtain a better error estimate for the static relative permittivity $\varepsilon_{r}$. However, this would only yield three independent results, and therefore a rather poor statistical error estimate. In order to improve error estimation, we can make use of the fact that the recorded current $\boldsymbol{J}(t)$ is a vector with three independent components, so that its autocorrelation is the sum of the individual components' autocorrelations

$$
\langle\boldsymbol{J}(0) \boldsymbol{J}(\tau)\rangle=\sum_{d \in\{x, y, z\}}\left\langle J_{d}(0) J_{d}(\tau)\right\rangle .
$$

Since the system under consideration is isotropic, the spatial components $\left\langle J_{d}(0) J_{d}(\tau)\right\rangle$ must be identical. However, this assumption does not strictly hold for the statistical estimates we are computing from a finite number of samples during a finite simulation time. Thus, together with that fact that $J_{x}, J_{y}, J_{z}$ are statistically independent, we can obtain three independent estimates of $\left\langle(\Delta \boldsymbol{M})^{2}(t)\right\rangle$ from each simulation run according to

$$
\left\langle(\Delta \boldsymbol{M})^{2}(t)\right\rangle=3\left\langle\left(\Delta M_{d}\right)^{2}(t)\right\rangle=6 t \int_{0}^{t}\left\langle J_{d}(0) J_{d}(\tau)\right\rangle \mathrm{d} \tau-6 \int_{0}^{t} \tau\left\langle J_{d}(0) J_{d}(\tau)\right\rangle \mathrm{d} \tau .
$$

Thus, we have nine independent estimates of $\left\langle(\Delta \boldsymbol{M})^{2}(t)\right\rangle$ available for error analysis. These estimates are displayed in fig. S3.

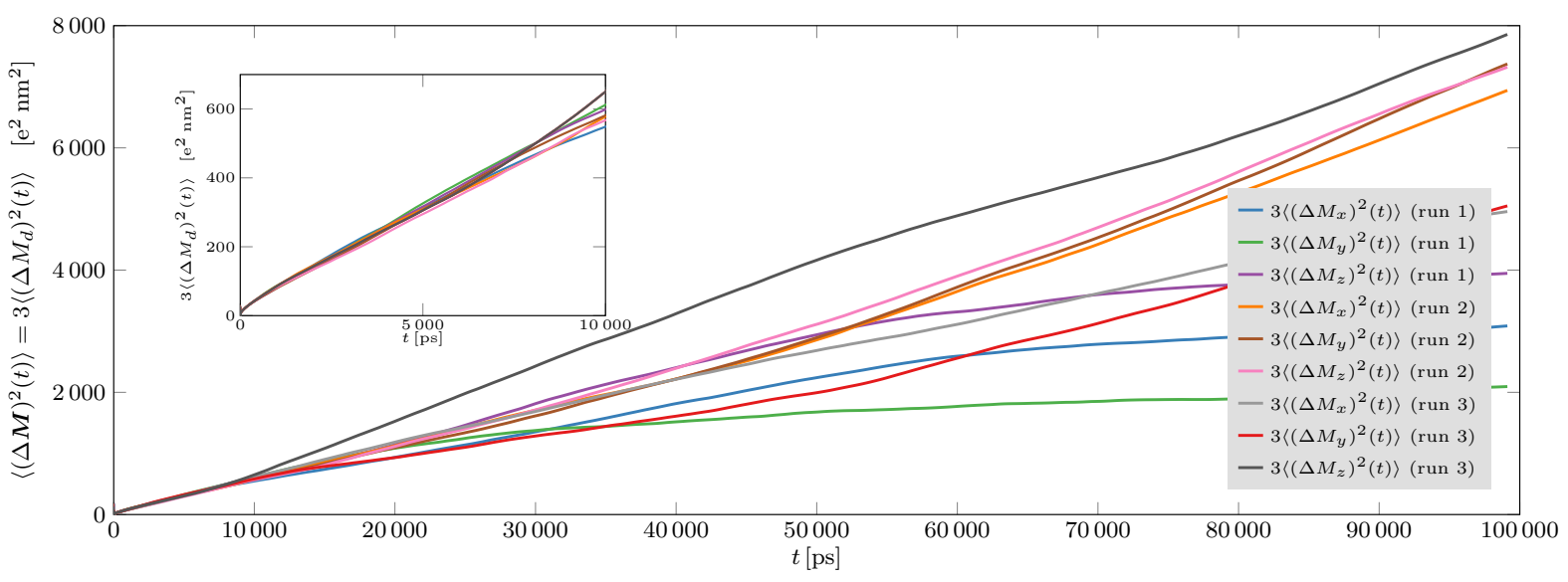

Figure S3: Estimates of the mean square displacement $\left\langle(\Delta M)^{2}(t)\right\rangle$ of the system's itinerant dipole moment $\boldsymbol{M}$ obtained from separate analyses of the three spatial components of each of three independent simulation runs. Inset: Magnification of the first 10000 ps.

It is now obvious that the seemingly perfect linear behavior of $\left\langle(\Delta \boldsymbol{M})^{2}(t)\right\rangle$ is the average of many components, which are actually distributed all over the place for large $t$. However, we also see that for $3000 \leqslant t \leqslant 8000 \mathrm{ps}$, all traces follow approximately the same linear trend, which can also be seen in the magnification of this region (inset in fig. S3). Since the components are statistically independent, we can use them to estimate the standard error $\epsilon\left(\left\langle(\Delta \boldsymbol{M})^{2}(t)\right\rangle\right)$ for each sampled time $t$. Figure S4 displays $\left\langle(\Delta \boldsymbol{M})^{2}(t)\right\rangle$ (blue line) together with its corresponding statistical one-sigma error margin $\left\langle(\Delta \boldsymbol{M})^{2}(t)\right\rangle \pm \epsilon\left(\left\langle(\Delta \boldsymbol{M})^{2}(t)\right\rangle\right)$ (light blue area). Since for large $t$, the statistical error is indeed very large and the individual components do not follow a general linear trend anymore, one should refrain from using this data for fitting. 


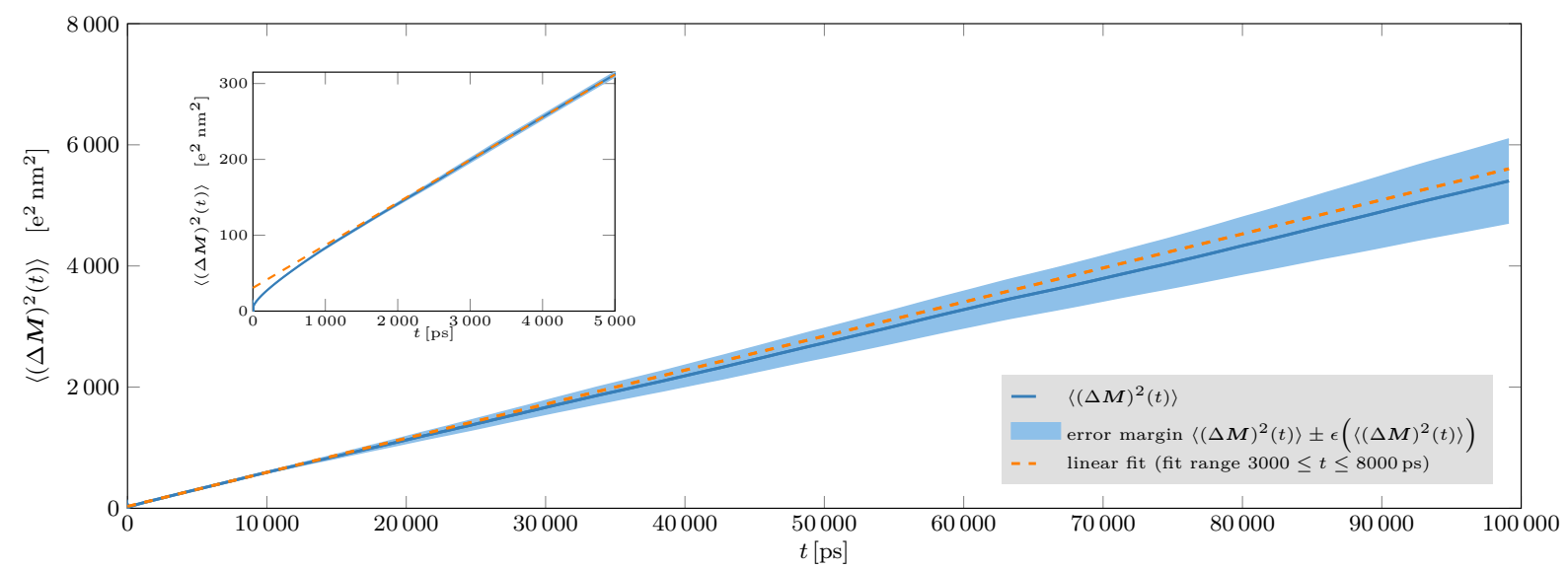

Figure S4: Mean square displacement $\left\langle(\Delta M)^{2}(t)\right\rangle$ (blue line) of the system's itinerant dipole moment $M$ with corresponding one-sigma error margin (light blue area). Inset: Magnification of the first $5000 \mathrm{ps}$.

The start of the fit range must be chosen according to the time where the integrated current autocorrelation converges to a constant (here: $t \approx 3000 \mathrm{ps}$ ), and its end according to the time where the individual components $3\left\langle\left(\Delta M_{d}\right)^{2}(t)\right\rangle$ begin to deviate from the general linear trend (here: $t \approx 8000 \mathrm{ps}$ ). The resulting fit is shown as a dashed orange line in fig. S4.

At this point, it is important to note that we still have the problem of oversampled data. Thus, the statistical error still cannot be inferred from the fit covariance matrix even if $\epsilon\left(\left\langle(\Delta \boldsymbol{M})^{2}(t)\right\rangle\right)$ is taken into account in the fitting procedure. Instead, since all components show the same linear trend in the fit range, the error $\epsilon\left(\left\langle(\Delta \boldsymbol{M})^{2}(t)\right\rangle\right)$ can be entirely attributed to shifts in the amplitude, and thus, the true error of the fit parameter $c_{2}$ can be well approximated by the average value of $\epsilon\left(\left\langle(\Delta M)^{2}(t)\right\rangle\right)$ in the fit range.

The resulting fit parameter $c_{2}=36.6141 \pm 6.1947 \mathrm{e}^{2} \mathrm{~nm}^{2}$ now allows us to compute the relative permittivity with a reasonable error estimate, yielding

$$
\varepsilon_{r}=1+\frac{(36.6141 \pm 6.1947) \mathrm{e}^{2} \mathrm{~nm}^{2}}{6 \cdot(301.7629 \pm 0.0026) \mathrm{nm}^{3} \cdot \varepsilon_{0} \cdot k_{\mathrm{B}} \cdot 300 \mathrm{~K}}=15.15 \pm 2.39 .
$$

Compared to the "naive" result we obtained in eq. (S48), we see that taking the statistical error of $\left\langle(\Delta \boldsymbol{M})^{2}(t)\right\rangle$ into account does not only dramatically increase the statistical error of the permittivity estimate $\varepsilon_{r}$, it also affects its value quite substantially. Ultimately, we see that the quality of permittivity data obtained via the Einstein-Helfand method is rather questionable if no proper error estimation is performed. 


\section{S2 Additional Results}

\section{S2.1 Bulk systems}

\section{S2.1.1 Volumetric system properties}

\begin{tabular}{|c|c|c|c|c|}
\hline \multicolumn{5}{|c|}{ pure $\left[\mathrm{C}_{4} \mathrm{C}_{1} \mathrm{Im}\right]^{+}\left[\mathrm{PF}_{6}\right]^{-}$(all-atom) } \\
\hline$c(\mathrm{~mol} / \mathrm{l})$ & $\bar{V}\left(\mathrm{~nm}^{3}\right)$ & $\bar{\varrho}\left(\mathrm{kg} / \mathrm{m}^{3}\right)$ & $\varrho_{\exp }\left(\mathrm{kg} / \mathrm{m}^{3}\right)$ & $\Delta \varrho(\%)$ \\
\hline $4.62987(4)$ & $38,735.0(3)$ & $1,315.735(10)$ & $1,360.47$ & -3.29 \\
\hline \multicolumn{5}{|c|}{ pure $\left[\mathrm{C}_{4} \mathrm{C}_{1} \mathrm{Im}\right]^{+}\left[\mathrm{PF}_{6}\right]^{-}$(coarse-grained) } \\
\hline$c(\mathrm{~mol} / \mathrm{l})$ & $\bar{V}\left(\mathrm{~nm}^{3}\right)$ & $\bar{\varrho}\left(\mathrm{kg} / \mathrm{m}^{3}\right)$ & $\varrho_{\exp }\left(\mathrm{kg} / \mathrm{m}^{3}\right)$ & $\Delta \varrho(\%)$ \\
\hline $4.78280(2)$ & $124,396.7(5)$ & $1,359.223(6)$ & $1,360.47$ & -0.09 \\
\hline
\end{tabular}

\begin{tabular}{c|c|c|r|r}
\multicolumn{5}{c}{$\left[\mathrm{C}_{4} \mathrm{C}_{1} \mathrm{Im}^{+}\left[\mathrm{NTf}_{2}\right]^{-}\right.$in PC (all-atom) } \\
$c(\mathrm{~mol} / \mathrm{l})$ & $\bar{V}\left(\mathrm{~nm}^{3}\right)$ & $\bar{\varrho}\left(\mathrm{kg} / \mathrm{m}^{3}\right)$ & $\varrho$ exp $\left(\mathrm{kg} / \mathrm{m}^{3}\right)$ & $\Delta \varrho(\%)$ \\
\hline \hline $0.00000(0)$ & $139.46(5)$ & $1,215.5(4)$ & $1,197.30$ & 1.52 \\
$0.53340(1)$ & $622.628(5)$ & $1,258.31(1)$ & $1,229.92$ & 2.31 \\
$0.96600(1)$ & $859.490(1)$ & $1,292.67(1)$ & $1,262.49$ & 2.39 \\
$1.62504(1)$ & $510.922(1)$ & $1,345.08(1)$ & $1,307.08$ & 2.91 \\
$2.10316(1)$ & $394.772(3)$ & $1,383.12(1)$ & $1,339.01$ & 3.29 \\
$2.46649(2)$ & $336.619(3)$ & $1,412.06(1)$ & $1,363.58$ & 3.56 \\
$2.75140(2)$ & $301.763(3)$ & $1,434.72(1)$ & $1,382.19$ & 3.80 \\
$2.98136(3)$ & $278.487(3)$ & $1,452.98(2)$ & $1,396.75$ & 4.03 \\
$3.17022(4)$ & $261.896(3)$ & $1,467.99(2)$ & $1,408.63$ & 4.21 \\
$3.32780(4)$ & $249.495(3)$ & $1,480.49(2)$ & $1,418.32$ & 4.38 \\
$3.46103(7)$ & $239.891(5)$ & $1,491.00(3)$ & $1,426.15$ & 4.55 \\
$3.57732(5)$ & $232.092(3)$ & $1,500.20(2)$ & $1,432.52$ & 4.72
\end{tabular}

$\mathrm{NaCl}$ in $\mathrm{SPC} / \mathrm{E}$ water (all-atom)

\begin{tabular}{l|c|r|r|r}
$c(\mathrm{~mol} / \mathrm{l})$ & $\bar{V}\left(\mathrm{~nm}^{3}\right)$ & $\bar{\varrho}\left(\mathrm{kg} / \mathrm{m}^{3}\right)$ & $\varrho_{\exp }\left(\mathrm{kg} / \mathrm{m}^{3}\right)$ & $\Delta \varrho(\%)$ \\
\hline \hline $1.155534(2)$ & $5,388.87(1)$ & $1,047.766(2)$ & $1,042.34$ & 0.52 \\
$2.432431(8)$ & $2,559.999(8)$ & $1,098.209(4)$ & $1,090.56$ & 0.70 \\
$4.433304(9)$ & $80,905.0(2)$ & $1,168.069(2)$ & $1,162.79$ & 0.45 \\
$4.86353(2)$ & $1,280.349(5)$ & $1,181.711(5)$ & $1,178.07$ & 0.31 \\
$5.19117(3)$ & $1,199.541(7)$ & $1,191.838(7)$ & $1,189.70$ & 0.18
\end{tabular}

Table S1: Volumetric system properties. The listed values are molar ion pair concentration $c(\mathrm{~mol} / \mathrm{I})$, average simulation box volume $\bar{V}\left(\mathrm{~nm}^{3}\right)$, average mass density $\varrho\left(\mathrm{kg} / \mathrm{m}^{3}\right)$, experimental mass density $\varrho \exp \left(\mathrm{kg} / \mathrm{m}^{3}\right)$, and relative deviation $\Delta \varrho=\frac{\bar{\varrho}-\varrho_{\exp }}{\varrho}(\%)$. The experimental value of $\varrho$ exp for $\left[\mathrm{C}_{4} \mathrm{C}_{1} \mathrm{Im}\right]^{+}\left[\mathrm{PF}_{6}\right]^{-}$at $300 \mathrm{~K}$ is a linear extrapolation of temperaturedependent mass density measurements taken from Machida et al., ${ }^{\mathrm{S} 3}$ whereas the values of $\varrho_{\exp }$ for $\left[\mathrm{C}_{4} \mathrm{C}_{1} \mathrm{Im}\right]^{+}\left[\mathrm{NTf}_{2}\right]^{-}$in PC are linear interpolations of temperature- and concentration-dependent measurements obtained from Vraneš et al. ${ }^{\mathrm{S} 4}$ For aqueous $\mathrm{NaCl}$, the values of $\varrho$ exp are linear inter- and extrapolations of temperature- and concentration-dependent data from ref. S5. 


\section{S2.1.2 Static relative dielectric permittivities}

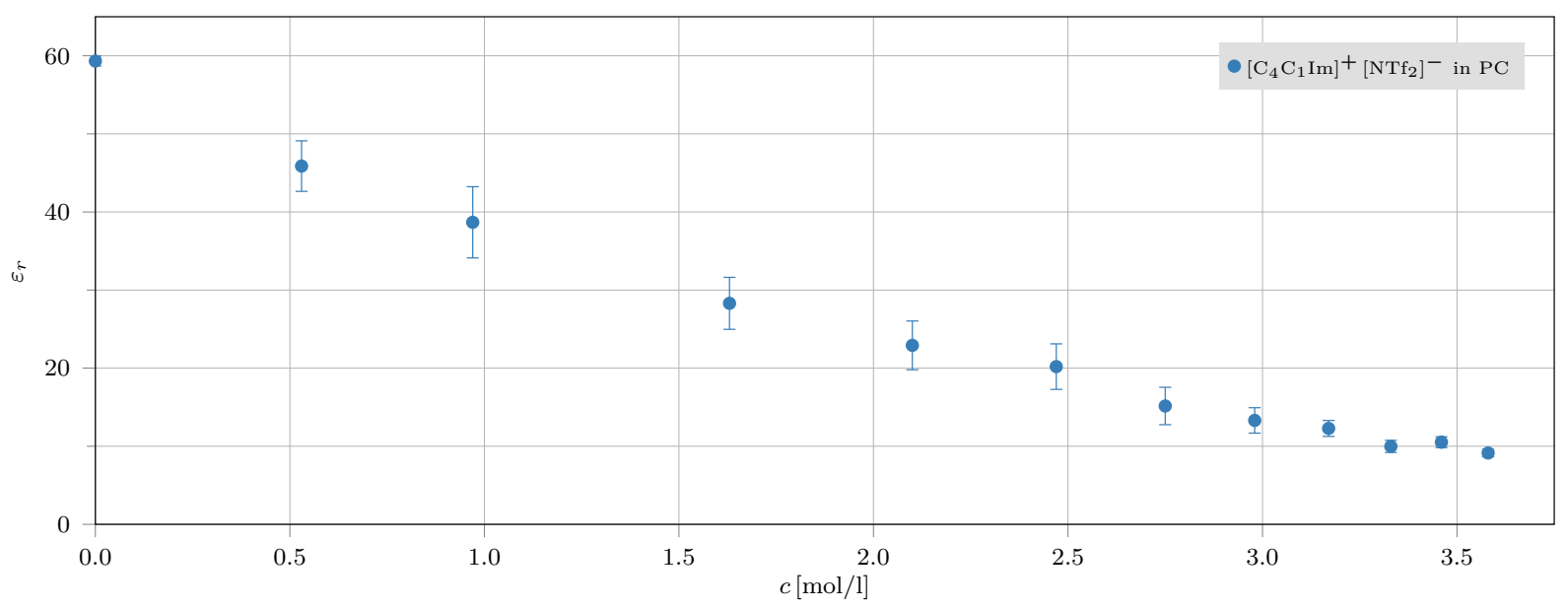

Figure S5: Static relative dielectric permittivities $\varepsilon_{r}$ of $\left[\mathrm{C}_{4} \mathrm{C}_{1} \mathrm{Im}\right]^{+}\left[\mathrm{NTf}_{2}\right]^{-}$in $\mathrm{PC}$ as a function of molar concentration $c$ according to table S2.

\begin{tabular}{l|r|l|l|l}
\multicolumn{6}{c}{$\left[\mathrm{C}_{4} \mathrm{C}_{1} \mathrm{Im}\right]^{+}\left[\mathrm{NTf}_{2}\right]^{-}$in PC } \\
$x$ & $c(\mathrm{~mol} / \mathrm{l})$ & $\varepsilon_{r}$ & \multicolumn{1}{c}{$\lambda_{\mathrm{D}}(\mathrm{nm})$} & $\lambda_{\mathrm{S}}(\mathrm{nm})$ \\
\hline \hline 0.00 & 0.00 & $59.3(6)$ & - & - \\
0.05 & 0.53 & $46(3)$ & $0.32(1)$ & $0.67(3)$ \\
0.10 & 0.97 & $39(5)$ & $0.22(1)$ & $0.65(4)$ \\
0.20 & 1.63 & $28(3)$ & $0.144(8)$ & $0.496(8)$ \\
0.30 & 2.10 & $23(3)$ & $0.114(8)$ & $0.465(6)$ \\
0.40 & 2.47 & $20(3)$ & $0.099(7)$ & $0.584(9)$ \\
0.50 & 2.75 & $15(2)$ & $0.081(6)$ & $0.607(4)$ \\
0.60 & 2.98 & $13(2)$ & $0.073(4)$ & $0.686(4)$ \\
0.70 & 3.17 & $12(1)$ & $0.068(3)$ & $0.753(6)$ \\
0.80 & 3.33 & $10.0(8)$ & $0.060(2)$ & $0.846(8)$ \\
0.90 & 3.46 & $10.5(7)$ & $0.060(2)$ & $0.95(1)$ \\
1.00 & 3.58 & $9.1(5)$ & $0.055(1)$ & $1.07(1)$
\end{tabular}

Table S2: Static relative permittivities $\varepsilon_{r}$, Debye lengths $\lambda_{\mathrm{D}}$, and effective screening lengths $\lambda_{\mathrm{S}}$ for solutions of $\left[\mathrm{C}_{4} \mathrm{C}_{1} \mathrm{Im}^{+}\right]^{+}\left[\mathrm{NT} \mathrm{f}_{2}\right]^{-}$in PC at different concentrations. The numbers in parentheses represent the uncertainty of the last digit. 


\section{S2.1.3 PMF behavior over time}

As stated in the main article, when analyzing simulations of concentrated ionic liquid systems, it is crucial that enough simulation time has been allowed for such systems to reach thermal equilibrium. The following plots show how the PMFs of the largest investigated systems (pure $\left[\mathrm{C}_{4} \mathrm{C}_{1} \mathrm{Im}\right]^{+}\left[\mathrm{PF}_{6}\right]^{-}$, AA and CG models) change over time.

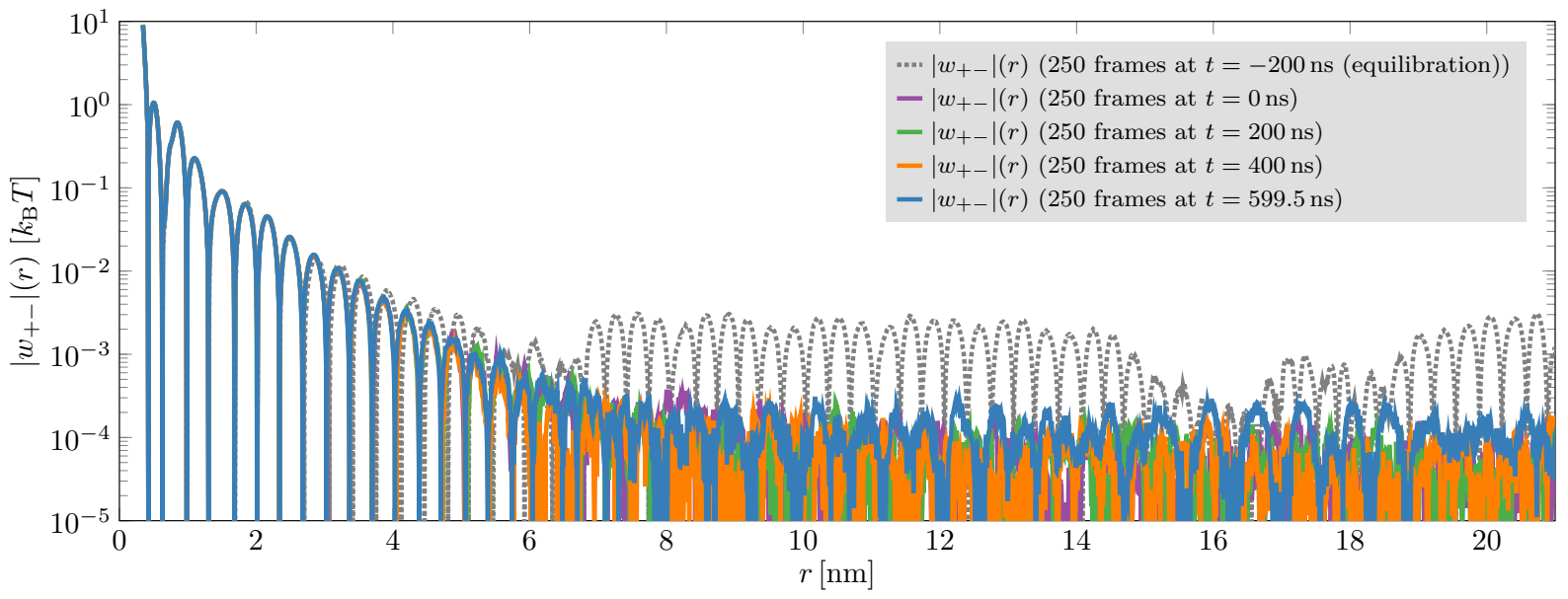

Figure S6: Absolute value of the potential of mean force $\left|w_{+-}\right|(r)$ between anions and cations in neat $\left[\mathrm{C}_{4} \mathrm{C}_{1} \operatorname{lm}\right]^{+}\left[\mathrm{PF}_{6}\right]^{-}(\mathrm{AA} \mathrm{model})$ for 250 analyzed frames (corresponding to a time span $\Delta t=0.5 \mathrm{~ns}$ ) taken at different time steps $t$ during the simulation The first PMF at $t=-200 \mathrm{~ns}$ (dashed gray line) was computed from trajectory data during system equilibration. Even though its short-range behavior is similar to the remaining PMF curves, this PMF exhibits comparatively large-amplitude, regular oscillations in its tail, showing that the system is not properly equilibrated at this point in time. The remaining PMFs were computed for the same number of frames but at times $t \in\{0,200,400,599.5\}$ ns after equilibration. After equilibration, all PMFs show the same qualitative behavior with rather noisy tails of similar amplitude, demonstrating that the system has reached an equilibrium state.

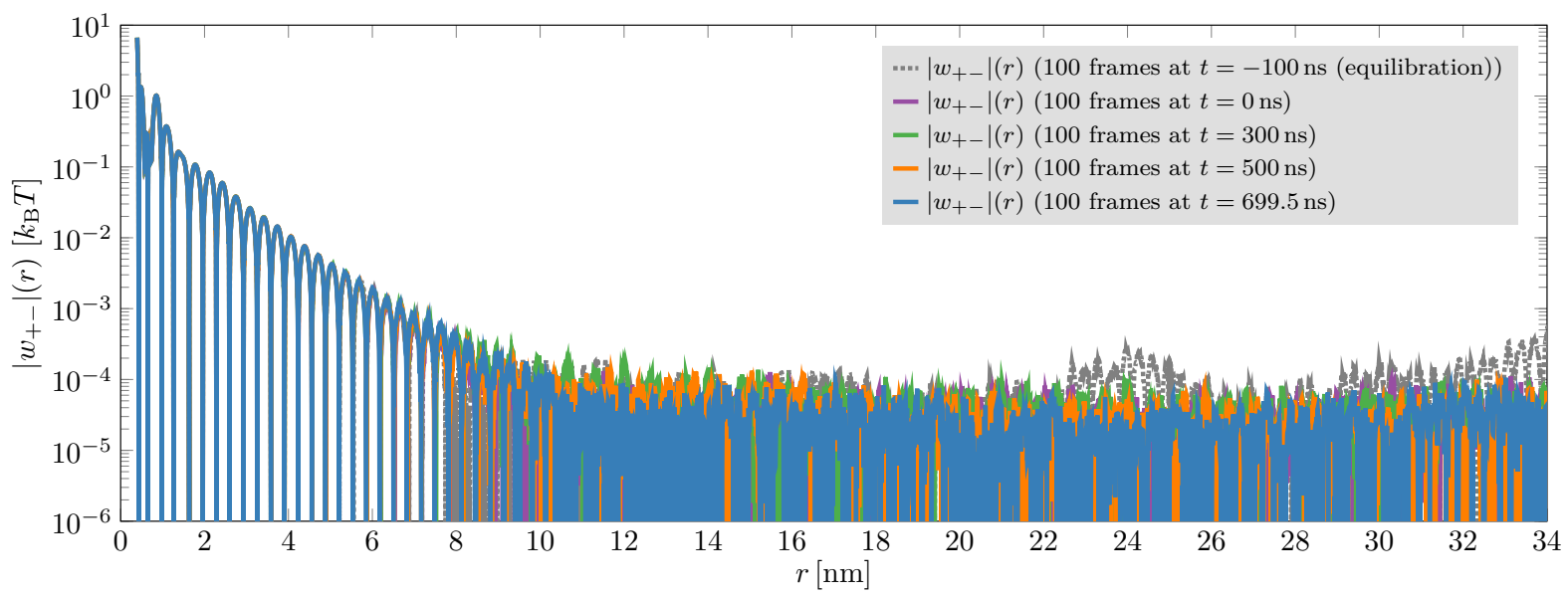

Figure S7: Absolute value of the potential of mean force $\left|w_{+-}\right|(r)$ between anions and cations in neat $\left[\mathrm{C}_{4} \mathrm{C}_{1} \mathrm{Im}\right]^{+}\left[\mathrm{PF}_{6}\right]^{-}(\mathrm{CG}$ model $)$ for 100 analyzed frames (corresponding to a time span $\Delta t=0.5 \mathrm{ns)}$ taken at different time steps $t$ during the simulation. The first PMF at $t=-100 \mathrm{~ns}$ (dashed gray line) was computed from trajectory data during system equilibration. It is clearly visible that there are small structural artifacts in the PMF tail at $r \approx 24 \mathrm{~nm}$ and for $r>29 \mathrm{~nm}$, showing that the system is not yet equilibrated at this point in time. The remaining PMFs were computed for the same number of frames but at times $t \in\{0,300,500,699.5\}$ ns after equilibration. Obviously, the structural artifacts have vanished after equilibration, and all PMFs show the same qualitative behavior, demonstrating that the system is indeed equilibrated.

The PMFs shown in the above figs. S6 and S7 demonstrate that the systems are properly equilibrated, however, they do not yield implications about the relaxation of the cumulative PMF behavior over time. Therefore, the following figs. S8 and S9 show the PMFs' relaxation behavior depending on the 
number of analyzed frames after equilibration.

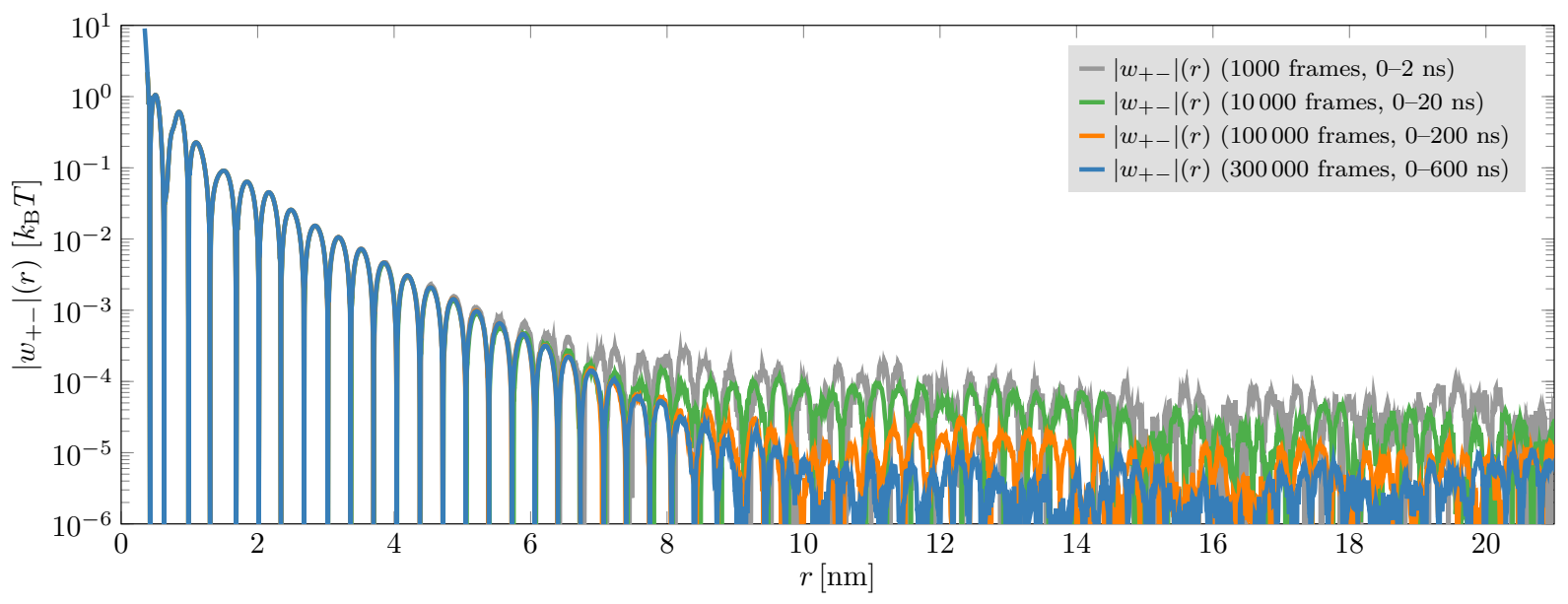

Figure S8: Absolute value of the potential of mean force $\left|w_{+-}\right|(r)$ between anions and cations in neat $\left[\mathrm{C}_{4} \mathrm{C}_{1} \mathrm{Im}\right]^{+}\left[\mathrm{PF}_{6}\right]^{-}(\mathrm{AA} \mathrm{model})$ for different numbers of analyzed frames (i.e., time spans). Regardless of the number of analyzed frames, the PMFs exhibit an exponentially damped oscillatory behavior, followed by rather noisy oscillations of almost constant amplitude. As the number of analyzed frames increases, the extent of the exponentially damped region increases to larger distances and the amplitude of the oscillatory tail decreases. However, there is no sign of any long-range monotonic decay.

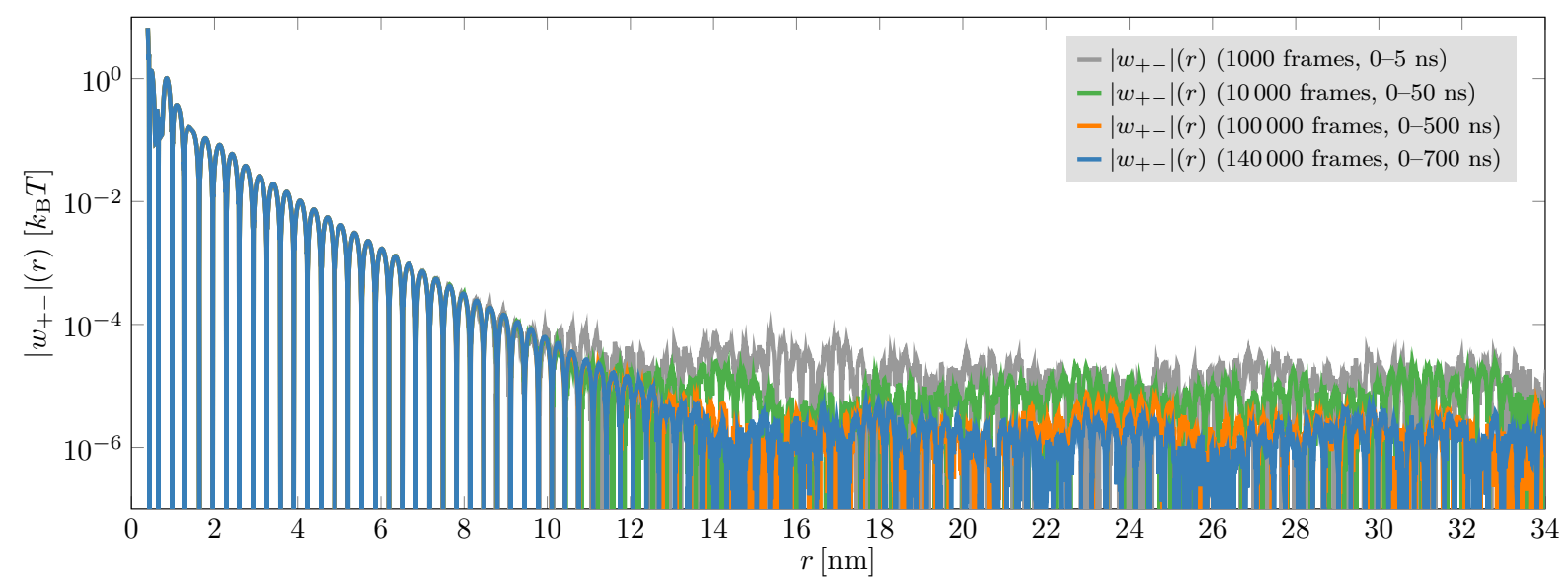

Figure S9: Absolute value of the potential of mean force $\left|w_{+-}\right|(r)$ between anions and cations in neat $\left[\mathrm{C}_{4} \mathrm{C}_{1} \mathrm{Im}\right]^{+}\left[\mathrm{PF}_{6}\right]^{-}\left(\mathrm{CG}^{-}\right.$model $)$ for different numbers of analyzed frames (i.e., time spans). As for the all-atom model, the PMFs exhibit an exponentially damped oscillatory behavior, followed by rather noisy oscillations of almost constant amplitude. Likewise, as the number of analyzed frames increases, the extent of the exponentially damped region increases to larger distances and the amplitude of the oscillatory tail decreases. Again, there is no sign of any long-range monotonic decay.

For both molecular models, the extent of the damped oscillatory region increases with simulation time but the qualitative PMF behavior remains unchanged and without any indication for a long-ranged, monotonic decay. 


\section{S2.1.4 Screening length scaling analysis of $\left[\mathrm{C}_{4} \mathrm{C}_{1} \mathrm{Im}\right]^{+}\left[\mathrm{NTf}_{2}\right]^{-}$in propylene carbonate}
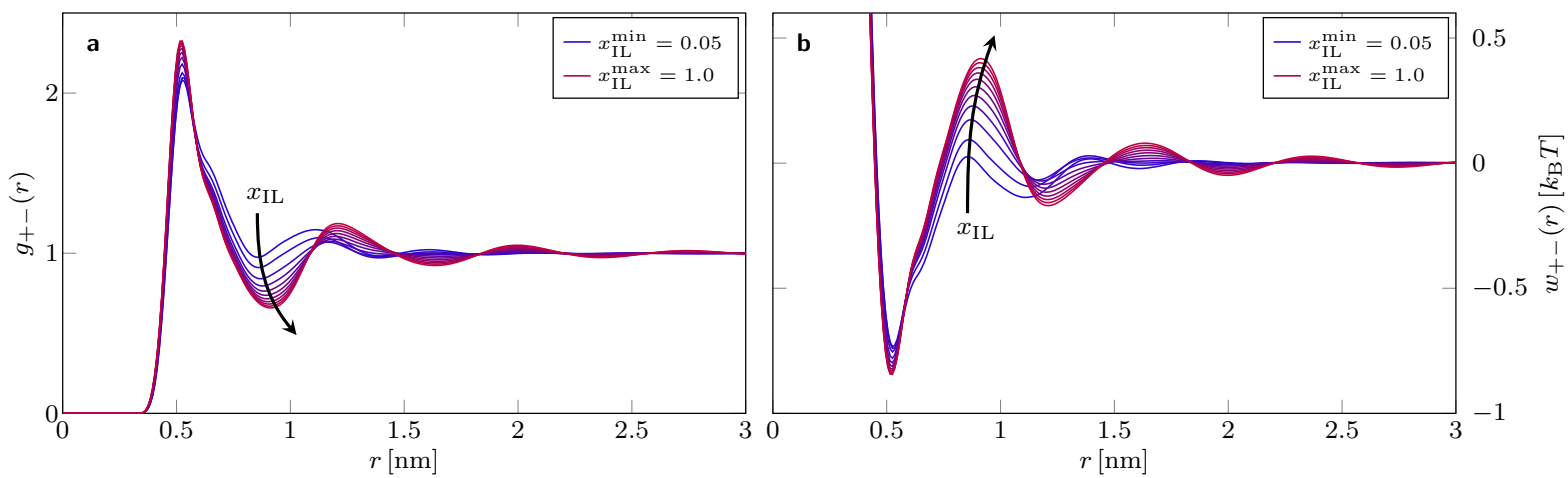

Figure S10: Panel a: Cation-anion radial distribution functions $g_{+-}(r)$ of $\left[\mathrm{C}_{4} \mathrm{C}_{1} \mathrm{Im}\right]^{+}\left[\mathrm{NTf}_{2}\right]^{-}$in propylene carbonate for different IL mole fractions $x$ ranging from $x=0.05$ (blue) to $x=1.0$ (red). Panel $\mathbf{b}$ : Corresponding cation-anion potentials of mean force $w_{+-}(r)$ of the same systems (same color code). Arrows indicate increasing IL mole fraction.
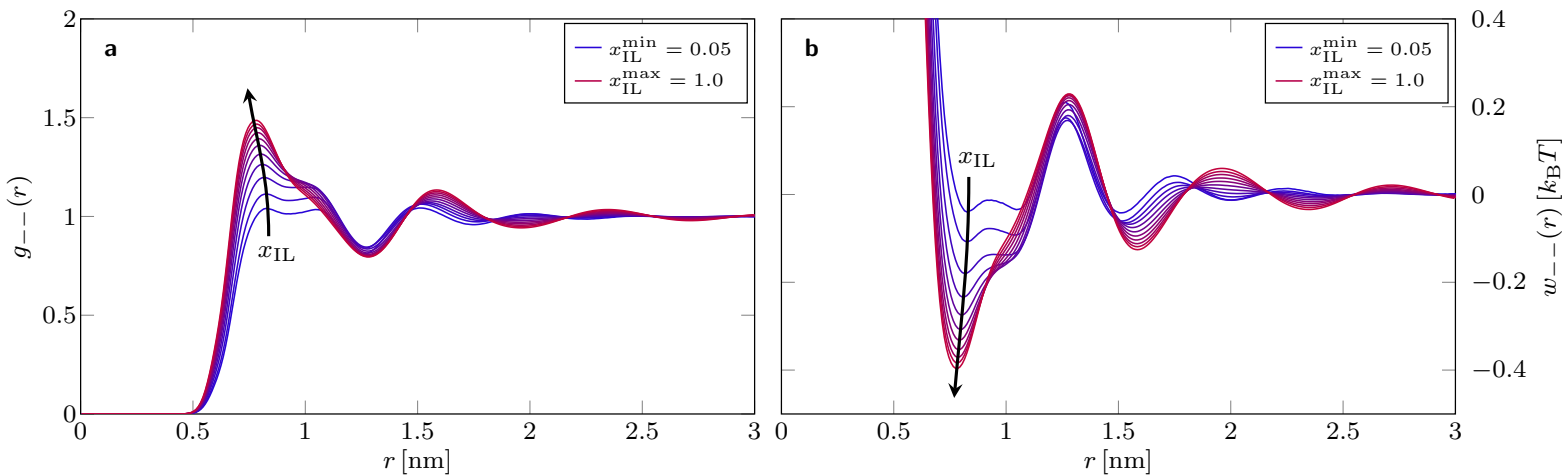

Figure S11: Panel a: Anion-anion radial distribution functions $g_{--}(r)$ of $\left[\mathrm{C}_{4} \mathrm{C}_{1} \mathrm{Im}\right]^{+}\left[\mathrm{NTf}_{2}\right]^{-}$in propylene carbonate for different IL mole fractions $x$ ranging from $x=0.05$ (blue) to $x=1.0$ (red). Panel $\mathbf{b}$ : Corresponding anion-anion potentials of mean force $w_{--}(r)$ of the same systems (same color code). Arrows indicate increasing IL mole fraction.
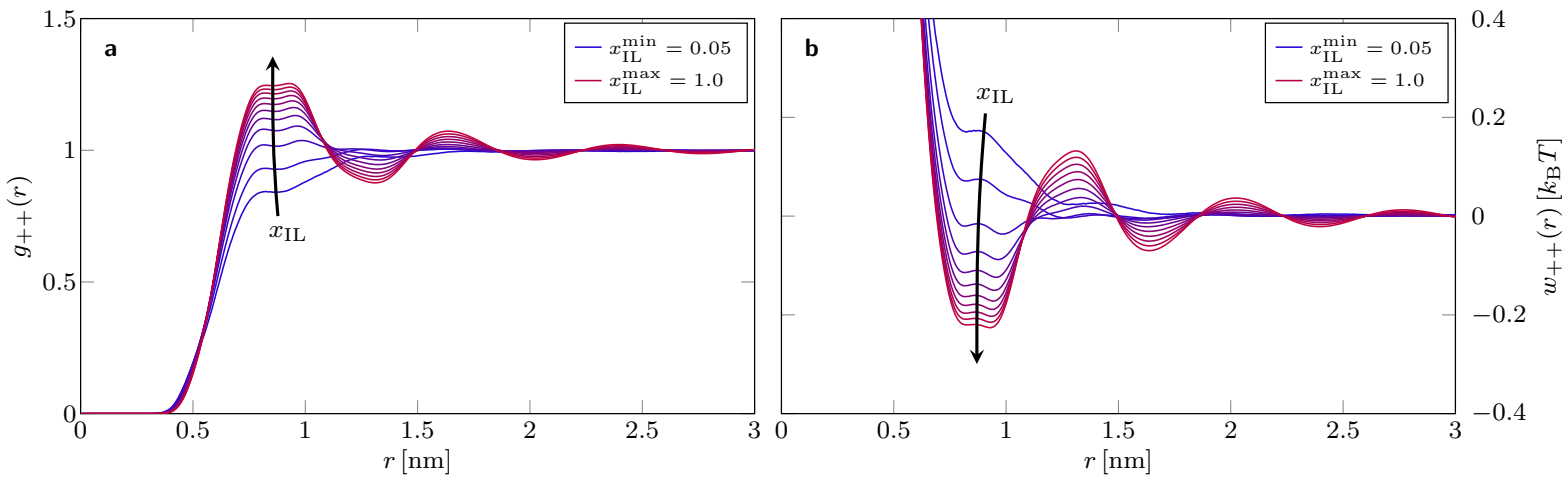

Figure S12: Panel a: Cation-cation radial distribution functions $g_{++}(r)$ of $\left[\mathrm{C}_{4} \mathrm{C}_{1} \mathrm{Im}\right]^{+}\left[\mathrm{NTf}_{2}\right]^{-}$in propylene carbonate for different IL mole fractions $x$ ranging from $x=0.05$ (blue) to $x=1.0$ (red). Panel $\mathbf{b}$ : Corresponding cation-cation potentials of mean force $w_{++}(r)$ of the same systems (same color code). Arrows indicate increasing IL mole fraction.

Listed below in tables S3 to S5 are the parameters resulting from fits of a superposition of $k=2$ 
oscillatory, exponentially damped functions of the form

$$
f(r)=\sum_{n=1}^{k} \frac{A_{n}}{r} \cos \left(\omega_{n} r-\phi_{n}\right) \exp \left(-\frac{r}{\lambda_{n}}\right)
$$

to the PMFs in solutions of $\left[\mathrm{C}_{4} \mathrm{C}_{1} \mathrm{Im}\right]^{+}\left[\mathrm{NTf}_{2}\right]^{-}$in propylene carbonate at different IL mole fractions $x$.

\begin{tabular}{r|r|r|l|r|r|c|c|c|c|c}
$x$ & $c_{\mathrm{IL}}(\mathrm{mol} / \mathrm{l})$ & $A_{1}\left(k_{\mathrm{B}} T\right)$ & $\lambda_{1}(\mathrm{~nm})$ & $2 \pi / \omega_{1}(\mathrm{~nm})$ & $\phi_{1}$ & $A_{2}\left(k_{\mathrm{B}} T\right)$ & $\lambda_{2}(\mathrm{~nm})$ & $2 \pi / \omega_{2}(\mathrm{~nm})$ & $\phi_{2}$ & $R^{2}$ \\
\hline \hline 0.05 & 0.53 & $1.11(4)$ & $0.444(5)$ & $0.477(1)$ & $5.83(5)$ & $-15(2)$ & $0.222(3)$ & $\infty$ & - & 0.9955 \\
0.10 & 0.97 & $1.13(7)$ & $0.404(6)$ & $0.458(1)$ & $0.38(7)$ & $9534(5496)$ & $0.158(7)$ & $116(1)$ & $2(44)$ & 0.9948 \\
0.20 & 1.63 & $0.39(2)$ & $0.496(8)$ & $0.465(3)$ & $5.87(5)$ & $2.8(2)$ & $0.309(5)$ & $0.799(3)$ & $6.04(6)$ & 0.9980 \\
0.30 & 2.10 & $0.79(8)$ & $0.41(1)$ & $0.468(6)$ & $5.6(1)$ & $1.6(1)$ & $0.395(7)$ & $0.729(3)$ & $1.37(7)$ & 0.9966 \\
0.40 & 2.47 & $1.16(9)$ & $0.373(7)$ & $0.465(5)$ & $5.6(1)$ & $1.42(5)$ & $0.462(4)$ & $0.731(1)$ & $1.56(3)$ & 0.9990 \\
0.50 & 2.75 & $1.5(1)$ & $0.348(6)$ & $0.461(5)$ & $5.84(9)$ & $1.14(2)$ & $0.555(3)$ & $0.7383(8)$ & $1.50(2)$ & 0.9996 \\
0.60 & 2.98 & $1.7(1)$ & $0.339(6)$ & $0.464(5)$ & $5.73(9)$ & $1.02(1)$ & $0.636(3)$ & $0.7409(6)$ & $1.45(1)$ & 0.9997 \\
0.70 & 3.17 & $1.5(1)$ & $0.351(6)$ & $0.467(5)$ & $5.55(9)$ & $0.99(1)$ & $0.700(3)$ & $0.7446(5)$ & $1.35(1)$ & 0.9998 \\
0.80 & 3.33 & $1.6(2)$ & $0.343(8)$ & $0.468(7)$ & $5.5(1)$ & $0.90(1)$ & $0.786(4)$ & $0.7457(5)$ & $1.32(1)$ & 0.9998 \\
0.90 & 3.46 & $1.6(2)$ & $0.34(1)$ & $0.468(8)$ & $5.53(1)$ & $0.83(1)$ & $0.871(5)$ & $0.7471(5)$ & $1.28(1)$ & 0.9997 \\
1.00 & 3.58 & $2.0(3)$ & $0.33(1)$ & $0.48(1)$ & $5.1(2)$ & $0.81(1)$ & $0.941(7)$ & $0.7457(5)$ & $1.28(1)$ & 0.9997
\end{tabular}

Table S3: Fit parameters of eq. (S52) to cation-anion PMFs $w_{+-}(r)$ in solutions of $\left[\mathrm{C}_{4} \mathrm{C}_{1} \mathrm{Im}\right]^{+}[\mathrm{NTf}]^{-}$in propylene carbonate for different mole fractions $x$ in the fit range $1.2 \leqslant r \leqslant 3.0 \mathrm{~nm}$. The values (units) are IL mole fraction $x$, concentration $c(\mathrm{~mol} / \mathrm{I})$, amplitudes $A_{n}\left(k_{\mathrm{B}} T\right)$, wavelengths $2 \pi / \omega_{n}(\mathrm{~nm})$, phase shifts $\phi_{n}$, and the coefficient of determination $R^{2}$. The numbers in parentheses represent the uncertainty of the last digit.

\begin{tabular}{|c|c|c|c|c|c|c|c|c|c|c|}
\hline$x$ & $c_{\mathrm{IL}}(\mathrm{mol} / \mathrm{l})$ & $A_{1}\left(k_{\mathrm{B}} T\right)$ & $\lambda_{1}(\mathrm{~nm})$ & $2 \pi / \omega_{1}(\mathrm{~nm})$ & $\phi_{1}$ & $A_{2}\left(k_{\mathrm{B}} T\right)$ & $\lambda_{2}(\mathrm{~nm})$ & $2 \pi / \omega_{2}(\mathrm{~nm})$ & $\phi_{2}$ & $R^{2}$ \\
\hline 0.05 & 0.53 & $3.8(1)$ & $0.421(4)$ & $0.4843(7)$ & $4.09(3)$ & $4.3(4)$ & $0.308(7)$ & $\infty$ & - & 0.9976 \\
\hline 0.10 & 0.97 & $0.9(1)$ & $0.56(1)$ & $0.463(2)$ & $5.4(1)$ & $88(10)$ & $0.201(4)$ & $0.489(4)$ & $3.7(1)$ & 0.9995 \\
\hline 0.20 & 1.63 & $3.8(2)$ & $0.389(5)$ & $0.475(3)$ & $4.61(7)$ & $1.9(2)$ & $0.36(1)$ & $0.714(5)$ & $4.8(1)$ & 0.9991 \\
\hline 0.30 & 2.10 & $3.5(2)$ & $0.382(4)$ & $0.469(3)$ & $4.94(5)$ & $1.75(5)$ & $0.465(6)$ & $0.720(2)$ & $4.82(4)$ & 0.9996 \\
\hline 0.40 & 2.47 & $3.5(1)$ & $0.373(4)$ & $0.466(2)$ & $5.16(5)$ & $1.69(5)$ & $0.522(4)$ & $0.723(1)$ & $4.79(2)$ & 0.9998 \\
\hline 0.50 & 2.75 & $3.8(2)$ & $0.358(4)$ & $0.466(3)$ & $5.24(5)$ & $1.40(3)$ & $0.607(4)$ & $0.7282(7)$ & $4.70(2)$ & 0.9998 \\
\hline 0.60 & 2.98 & $4.0(2)$ & $0.349(4)$ & $0.464(3)$ & $5.37(5)$ & $1.23(2)$ & $0.686(4)$ & $0.7310(7)$ & $4.63(1)$ & 0.9998 \\
\hline 0.70 & 3.17 & $5.0(4)$ & $0.329(5)$ & $0.464(4)$ & $5.46(8)$ & $1.13(2)$ & $0.753(6)$ & $0.7342(7)$ & $4.54(2)$ & 0.9997 \\
\hline 0.80 & 3.33 & $6.3(6)$ & $0.312(6)$ & $0.468(6)$ & $5.4(1)$ & $0.98(2)$ & $0.846(8)$ & $0.7371(8)$ & $4.46(1)$ & 0.9996 \\
\hline 0.90 & 3.46 & $7.5(9)$ & $0.303(7)$ & $0.476(7)$ & $5.1(1)$ & $0.85(2)$ & $0.95(1)$ & $0.7394(8)$ & $4.40(2)$ & 0.9995 \\
\hline 1.00 & 3.58 & $8(1)$ & $0.296(8)$ & $0.479(8)$ & $5.0(1)$ & $0.76(2)$ & $1.07(1)$ & $0.7388(9)$ & $4.38(2)$ & 0.9994 \\
\hline
\end{tabular}

Table S4: Fit parameters of eq. (S52) to anion-anion PMFs $w_{--}(r)$ in solutions of $\left.\left[\mathrm{C}_{4} \mathrm{C}_{1} \mathrm{Im}\right]^{+}[\mathrm{NTf}]_{2}\right]^{-}$in propylene carbonate for different mole fractions $x$ in the fit range $1.2 \leqslant r \leqslant 3.0 \mathrm{~nm}$. The values (units) are IL mole fraction $x$, concentration $c(\mathrm{~mol} / \mathrm{I})$, amplitudes $A_{n}\left(k_{\mathrm{B}} T\right)$, wavelengths $2 \pi / \omega_{n}(\mathrm{~nm})$, phase shifts $\phi_{n}$, and the coefficient of determination $R^{2}$. The numbers in parentheses represent the uncertainty of the last digit.

\begin{tabular}{|c|c|c|c|c|c|c|c|c|c|c|}
\hline$x$ & $c_{\mathrm{IL}}(\mathrm{mol} / \mathrm{l})$ & $A_{1}\left(k_{\mathrm{B}} T\right)$ & $\lambda_{1}(\mathrm{~nm})$ & $2 \pi / \omega_{1}(\mathrm{~nm})$ & $\phi_{1}$ & $A_{2}\left(k_{\mathrm{B}} T\right)$ & $\lambda_{2}(\mathrm{~nm})$ & $2 \pi / \omega_{2}(\mathrm{~nm})$ & $\phi_{2}$ & $R^{2}$ \\
\hline$\overline{0.05}$ & 0.53 & $0.09(1)$ & $0.67(3)$ & $0.47(1)$ & $\overline{00.7(1)}$ & $2.8(2)$ & $0.302(4)$ & $\infty$ & - & 0.9853 \\
\hline 0.10 & 0.97 & $0.07(1)$ & $0.65(4)$ & $0.46(2)$ & $1.0(1)$ & $0.21(6)$ & $0.43(3)$ & $1.11(2)$ & $6.0(2)$ & 0.8456 \\
\hline 0.20 & 1.63 & $0.24(3)$ & $0.46(1)$ & $0.48(1)$ & $5.5(1)$ & $0.18(3)$ & $0.44(2)$ & $0.96(2)$ & $0.3(2)$ & 0.9868 \\
\hline 0.30 & 2.10 & $56(25)$ & $0.19(1)$ & $0.53(2)$ & $4.3(3)$ & $0.3(1)$ & $0.45(3)$ & $0.86(1)$ & $1.6(2)$ & 0.9905 \\
\hline 0.40 & 2.47 & $12(2)$ & $0.226(6)$ & $0.50(1)$ & $5.0(2)$ & $0.26(2)$ & $0.58(1)$ & $0.740(3)$ & $4.77(7)$ & 0.9990 \\
\hline 0.50 & 2.75 & $92(34)$ & $0.169(7)$ & $0.45(2)$ & $0.9(4)$ & $0.50(2)$ & $0.597(6)$ & $0.734(2)$ & $4.95(4)$ & 0.9991 \\
\hline 0.60 & 2.98 & $12(2)$ & $0.227(6)$ & $0.48(1)$ & $5.3(3)$ & $0.56(1)$ & $0.668(3)$ & $0.730(1)$ & $5.12(3)$ & 0.9997 \\
\hline 0.70 & 3.17 & $24(4)$ & $0.206(4)$ & $0.47(1)$ & $5.8(2)$ & $0.584(7)$ & $0.744(3)$ & $0.7394(5)$ & $4.89(1)$ & 0.9999 \\
\hline 0.80 & 3.33 & $40(7)$ & $0.193(4)$ & $0.46(1)$ & $0.1(3)$ & $0.606(6)$ & $0.815(3)$ & $0.7411(4)$ & $4.82(1)$ & 0.9999 \\
\hline 0.90 & 3.46 & $84(16)$ & $0.176(4)$ & $0.43(2)$ & $1.3(3)$ & $0.604(5)$ & $0.895(3)$ & $0.7444(4)$ & $4.72(1)$ & 0.9999 \\
\hline 1.00 & 3.58 & $104(22)$ & $0.174(4)$ & $0.43(2)$ & $1.6(3)$ & $0.594(4)$ & $0.983(3)$ & $0.7432(4)$ & $4.71(1)$ & 0.9999 \\
\hline
\end{tabular}

Table S5: Fit parameters of eq. (S52) to cation-cation PMFs $w_{++}(r)$ in solutions of $\left[\mathrm{C}_{4} \mathrm{C}_{1} \mathrm{Im}\right]^{+}\left[\mathrm{NTf}_{2}\right]^{-}$in propylene carbonate for different mole fractions $x$ in the fit range $1.2 \leqslant r \leqslant 3.0 \mathrm{~nm}$. The values (units) are IL mole fraction $x$, concentration $c(\mathrm{~mol} / \mathrm{I})$, amplitudes $A_{n}\left(k_{\mathrm{B}} T\right)$, wavelengths $2 \pi / \omega_{n}(\mathrm{~nm})$, phase shifts $\phi_{n}$, and the coefficient of determination $R^{2}$. The numbers in parentheses represent the uncertainty of the last digit.

Fitting the data to eq. (S52) with $k=3$ terms resulted in extremely unstable results under slight variation of initial fit parameters, while setting $k=1$ lead to large fitting errors. 


\section{S2.2 Confined ionic liquid systems}

\section{S2.2.1 Number density profiles}

To investigate the layered structure of the IL in the $z$-direction of the different confined systems, we computed the center-of-mass-based number densities of ions according to

$$
\rho_{n}^{ \pm}(z)=\left\langle\sum_{i} \delta\left(z-z_{i}^{\mathrm{com}}\right)\right\rangle
$$

where $\delta(\cdot)$ is the Dirac delta function, the operator $\langle\cdot\rangle$ denotes the canonical average, and the index $i$ runs over all molecules in the system with center-of-mass position $z_{i}^{\text {com }}$, respectively. For the numerical evaluation of eq. (S53), the system is discretized in the $z$-direction into $n$ slabs of width $l_{z}=L_{z} / n$, where $L_{z}$ is the length of the simulation box in the $z$-direction. The number density profile is then calculated according to

$$
\rho_{n}^{ \pm}(z)=\left\langle\frac{1}{L_{x} L_{y} l_{z}} \sum_{\substack{i \\ 2\left|z-z_{i}^{\text {com }}\right|<l_{z}}} 1\right\rangle,
$$

The top row of fig. S13 shows snapshots of the $x$-z-plane of all confined systems (from left to right: $\left.L_{z}=8.2 \mathrm{~nm}, L_{z}=9.9 \mathrm{~nm}, L_{z}=13.2 \mathrm{~nm}, L_{z}=14.8 \mathrm{~nm}\right)$. The corresponding center-of-mass-based number densities of $\left[\mathrm{C}_{4} \mathrm{C}_{1} \mathrm{Im}\right]^{+}$cations $\left(\rho_{n}^{+}(z)\right.$, blue $)$ and $\left[\mathrm{NTf}_{2}\right]^{-}$anions $\left(\rho_{n}^{-}(z)\right.$, red $)$ are displayed in the bottom row.
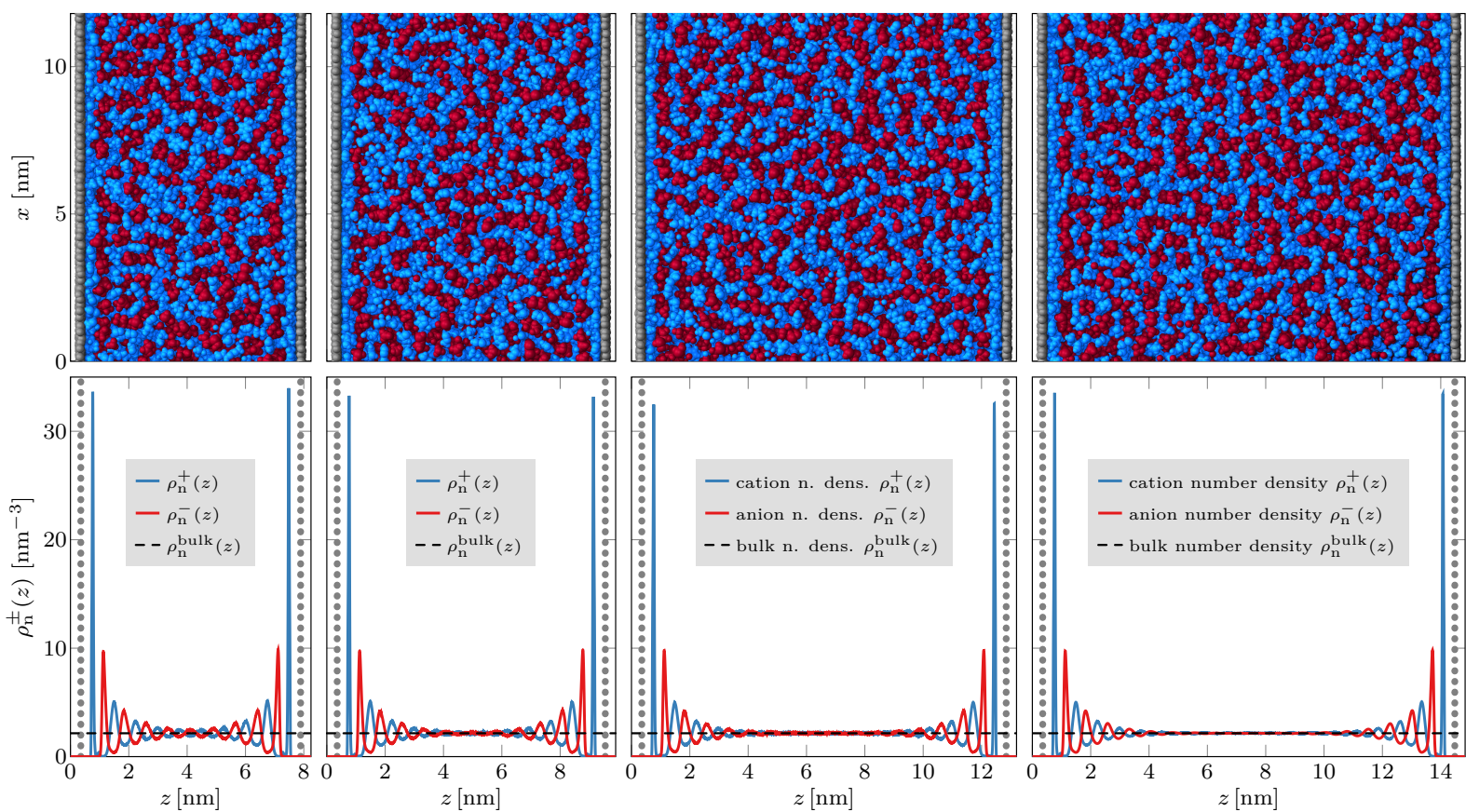

Figure S13: Top row: Snapshots of the $x$-z-plane of all confined systems with different simulation box sizes (from left to right: $\left.L_{z}=8.2 \mathrm{~nm}, L_{z}=9.9 \mathrm{~nm}, L_{z}=13.2 \mathrm{~nm}, L_{z}=14.8 \mathrm{~nm}\right)$. Bottom row: Cation $\left(\rho_{n}^{+}(z)\right.$, blue), anion $\left(\rho_{n}^{-}(z)\right.$, red), and bulk (black, dashed) number density $\rho_{n}(z)$ of $\left[\mathrm{C}_{4} \mathrm{C}_{1} \mathrm{Im}\right]^{+}\left[\mathrm{NTf}_{2}\right]^{-}$confined between like-charged graphene walls. In the vicinity of the walls (up to $z \approx 1 \mathrm{~nm}$, and likewise, down to $z \approx L_{z}-1 \mathrm{~nm}$ ), the total number density is almost entirely due to cations.

The ion number densities shown in fig. S13 make it clear that on either side of the systems, the first layer close to each of the negatively charged walls consists entirely of cations, all systems exhibit a very similar structure with alternating cation and anion layers, and this structural feature decays 
exponentially towards the center of the systems. A closer look at the number density profiles of the first three systems reveals that while all profiles decay in the same manner, the first cation layers close to the walls do not always have the exact same amplitude. This is a consequence of the fact that the total dipole moment of such slab systems fluctuates slowly around zero, and the average ion distributions have not fully converged to perfect symmetry within the simulation times of these systems $\left(t_{\mathrm{sim}}=1 \mu \mathrm{s}\right)$. The largest system (rightmost column in fig. S13), which is used for our analyses in the main article, does not suffer from this inaccuracy due to its much longer simulation time of $t_{\mathrm{sim}}=4 \mu \mathrm{s}$. This highlights the very slow dynamics in confined IL systems.

\section{S2.2.2 Charge density profiles}

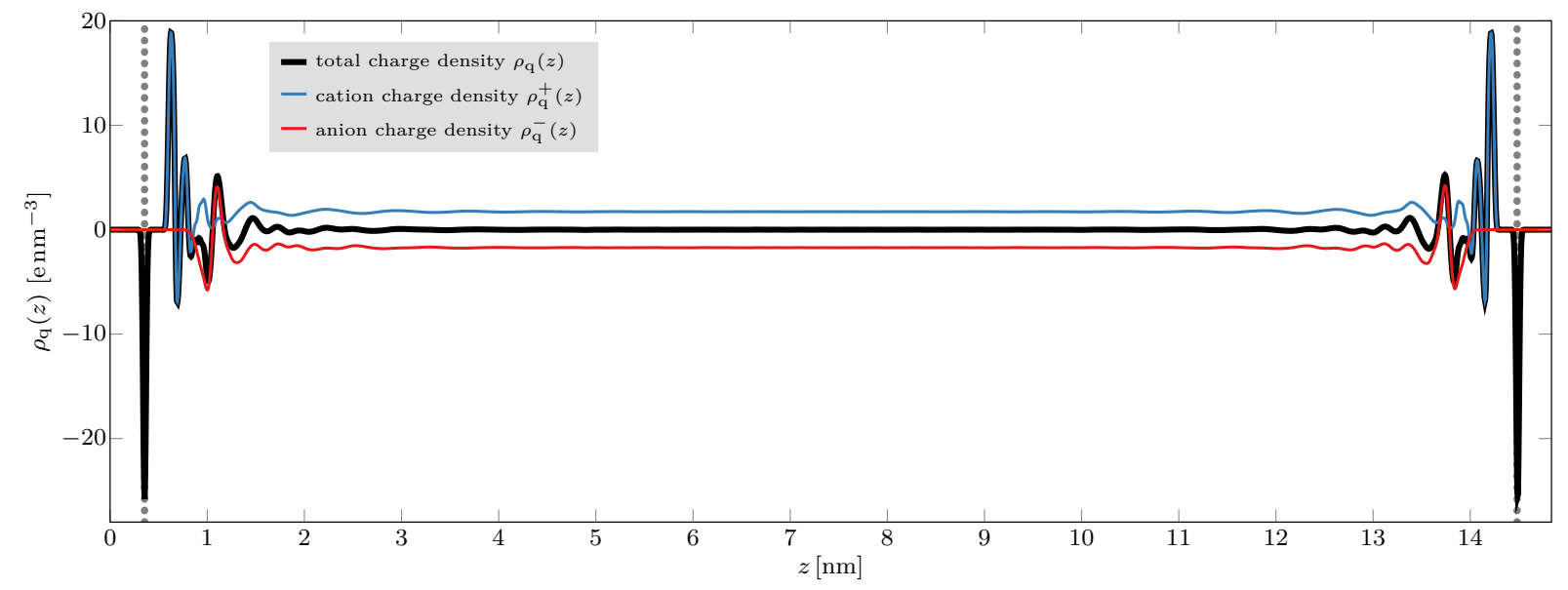

Figure S14: Cation $\left(\rho_{\mathrm{q}}^{+}(z)\right.$, blue), anion $\left(\rho_{\mathrm{q}}^{-}(z)\right.$, red), and total (black) charge density $\rho_{\mathrm{q}}(z)$ of $\left[\mathrm{C}_{4} \mathrm{C}_{1} \operatorname{lm}\right]^{+}[\mathrm{NTf}]^{-}$confined between like-charged graphene walls. In the vicinity of the walls (up to $z=0.81 \mathrm{~nm}$, and likewise, down to $z=L_{z}-0.81 \mathrm{~nm}$ ), the total charge density is entirely due to cations. Nevertheless, the charge density oscillates around zero in this region, which is due to the negative partial charges of carbon atoms in the cations' butyl and methyl side chains. Due to the typically preferred orientations of cations close the surface, these atoms have a high probability to be located at a specific distance from the surface, yielding a net-negative charge density in this region. Thereafter, between $z=0.81 \mathrm{~nm}$ and $z=1.2 \mathrm{~nm}$, the anion contribution dominates. For larger distances, both cations and anions have relatively similar contributions (in magnitude) to the total charge density. Towards the center of the system, all charge densities converge to their corresponding bulk values. 


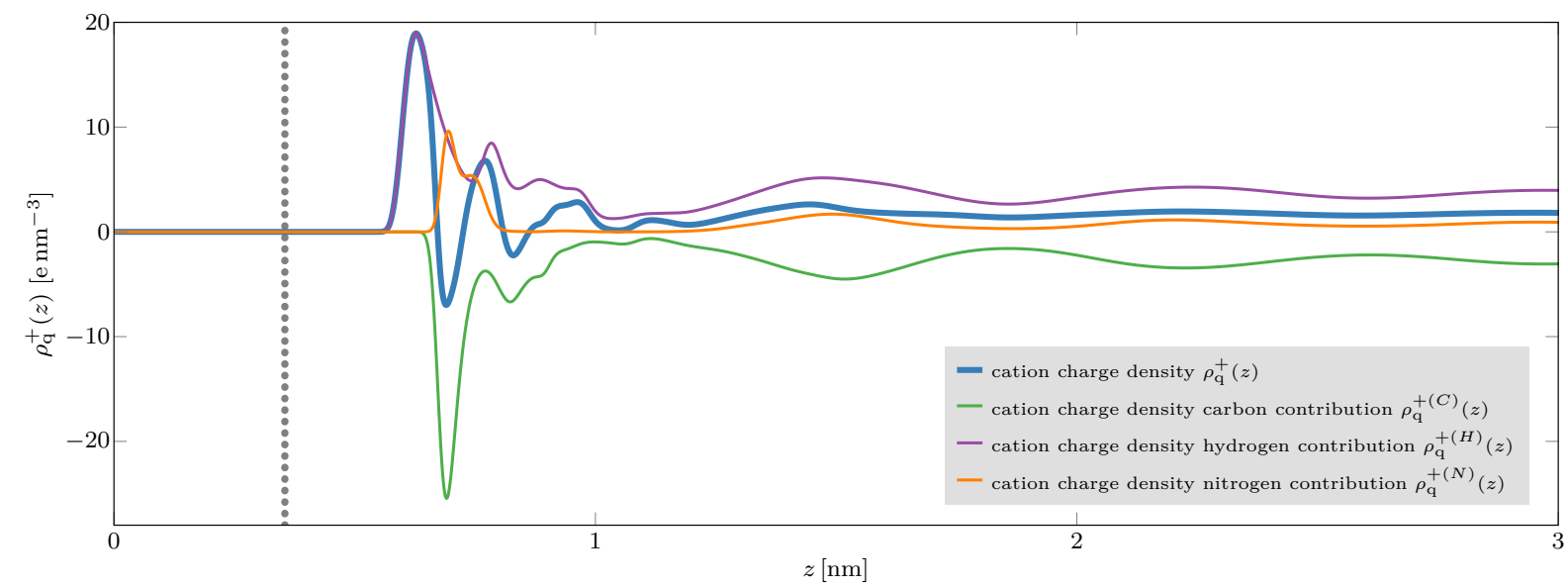

Figure S15: Different contributions to the cation charge density $\left(\rho_{\mathrm{a}}^{+}(z)\right.$ (thick blue line) of $\left[\mathrm{C}_{4} \mathrm{C}_{1} \mathrm{Im}\right]^{+}[\mathrm{NTf}]^{-}$confined between likecharged graphene walls. Close to the surface, the $\left[\mathrm{C}_{4} \mathrm{C}_{1} \mathrm{Im}\right]^{+}$ions are highly ordered so that their molecular structure affects the cumulative cation charge density. As a consequence, the first positive peak in $\rho_{\mathrm{q}}^{+}(z)$ at $z \approx 0.63 \mathrm{~nm}$ is entirely due to the contribution of hydrogen atoms $\rho_{\mathrm{q}}^{+(H)}(z)$ (purple line), and the negative peaks at $z \approx 0.69 \mathrm{~nm}$ and $z \approx 0.83 \mathrm{~nm}$ is caused by the contribution of carbon atoms $\rho_{\mathrm{q}}^{+(C)}(z)$ (green line).

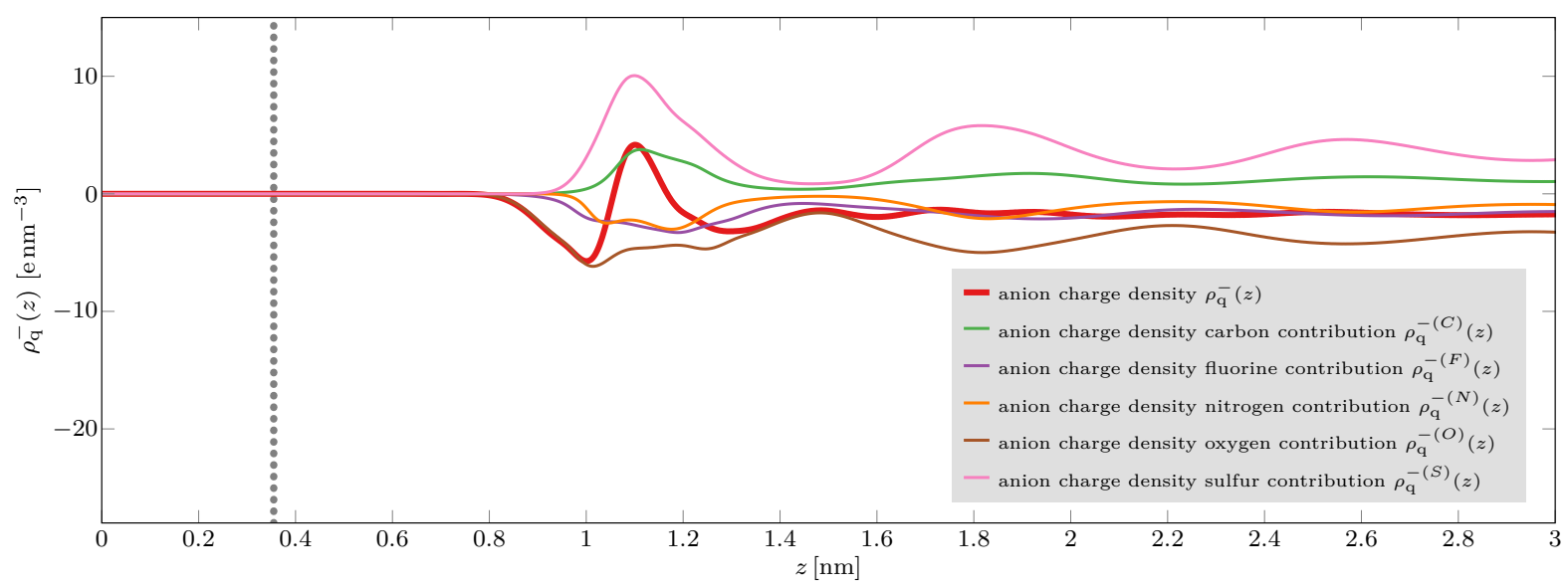

Figure S16: Different contributions to the anion charge density $\left(\rho_{\mathrm{q}}^{-}(z) \text { (red) of }\left[\mathrm{C}_{4} \mathrm{C}_{1} \mathrm{Im}\right]^{+}[\mathrm{NTf}]_{2}\right]^{-}$confined between like-charged graphene walls. The first negative peak in $\rho_{\mathrm{q}}^{-}(z)$ at $z \approx 1.0 \mathrm{~nm}$ is mostly due to the contribution of oxygen atoms $\rho_{\mathrm{q}}^{-(O)}(z)$ (brown line), and the positive peak at $z \approx 1.1 \mathrm{~nm}$ is caused by the contributions of carbon $\left(\rho_{\mathrm{q}}^{-(C)}(z)\right.$, green line) and of sulfur atoms $\left(\rho_{\mathrm{q}}^{-(S)}(z)\right.$, pink line). Thereafter, even though especially the oxygen and sulfur contributions still oscillate, the different contributions mostly cancel out so that the total anion charge density converges rather quickly to its bulk value.

\section{S2.2.3 Position-dependent RDFs}

Computing RDFs $g_{X Y}^{(i)}\left(r ; z_{i}\right)$ between a spatially selective group of species $X$ consisting only of molecules whose center of mass lies within a slab extending in the $x y$-plane and centered at $z=z_{i}$ with width $l_{z}$, and a second group of molecular species $Y$ without such constraints, requires slight modifications of eqs. (S14) and (S15). If the molecular species in $X$ are different from those in $Y$, i.e., the groups are disjoint, eq. (S14) has to read

$$
\hat{g}_{X Y}^{(i)}\left(r_{n}, z_{i}\right)=\frac{1}{N \bar{\rho}_{Y}^{\text {bulk }} V_{n}^{\text {shell }}} \sum_{m=1}^{N} \frac{1}{N_{X, i, m}} \sum_{j=1}^{N_{X, i, m}} \sum_{k=1}^{N_{Y}} \delta\left(\left\lfloor\frac{\left\|\boldsymbol{r}_{k, m}-\boldsymbol{r}_{j, m}\right\|_{\mathrm{pbc}}}{\Delta r}\right\rfloor-n\right),
$$


where $\bar{\rho}_{Y}^{\text {bulk }}$ is the average bulk number density of molecules of species $Y$, and the second sum runs over all $N_{X, i, m}$ molecules of species $X$ whose center of mass positions' $z$-coordinates $z_{m}^{\text {com }}$ fulfill the condition $2\left|z_{i}-z_{m}^{\text {com }}\right| \leqslant l_{z}$ at time step $m$.

If $X=Y$, the groups overlap (the first group is a subset of the second) so that one has to exclude distances of molecules with themselves. Since the RDF bin width $\Delta r$ is usually chosen much smaller than the smallest intermolecular distance, this can be accomplished by simply setting $\hat{g}_{X Y}^{(i)}\left(r_{n}, z_{i}\right)=0$ if $n=0$.

Shown below in fig. S17 are the results of $z$-position-dependent RDFs $g_{-+}^{(i)}\left(r ; z_{i}\right)$ (anion-cation), $g_{++}^{(i)}\left(r ; z_{i}\right)$ (cation-cation), and $g_{--}^{(i)}\left(r ; z_{i}\right)$ (anion-anion) computed in this manner, as were the cationanion RDFs displayed in section III.D. of the main article. The amplitudes of the first maxima and minima (middles and bottom row in fig. S17) decay with $z_{i}$ in a similar manner as the ones shown in the main article.
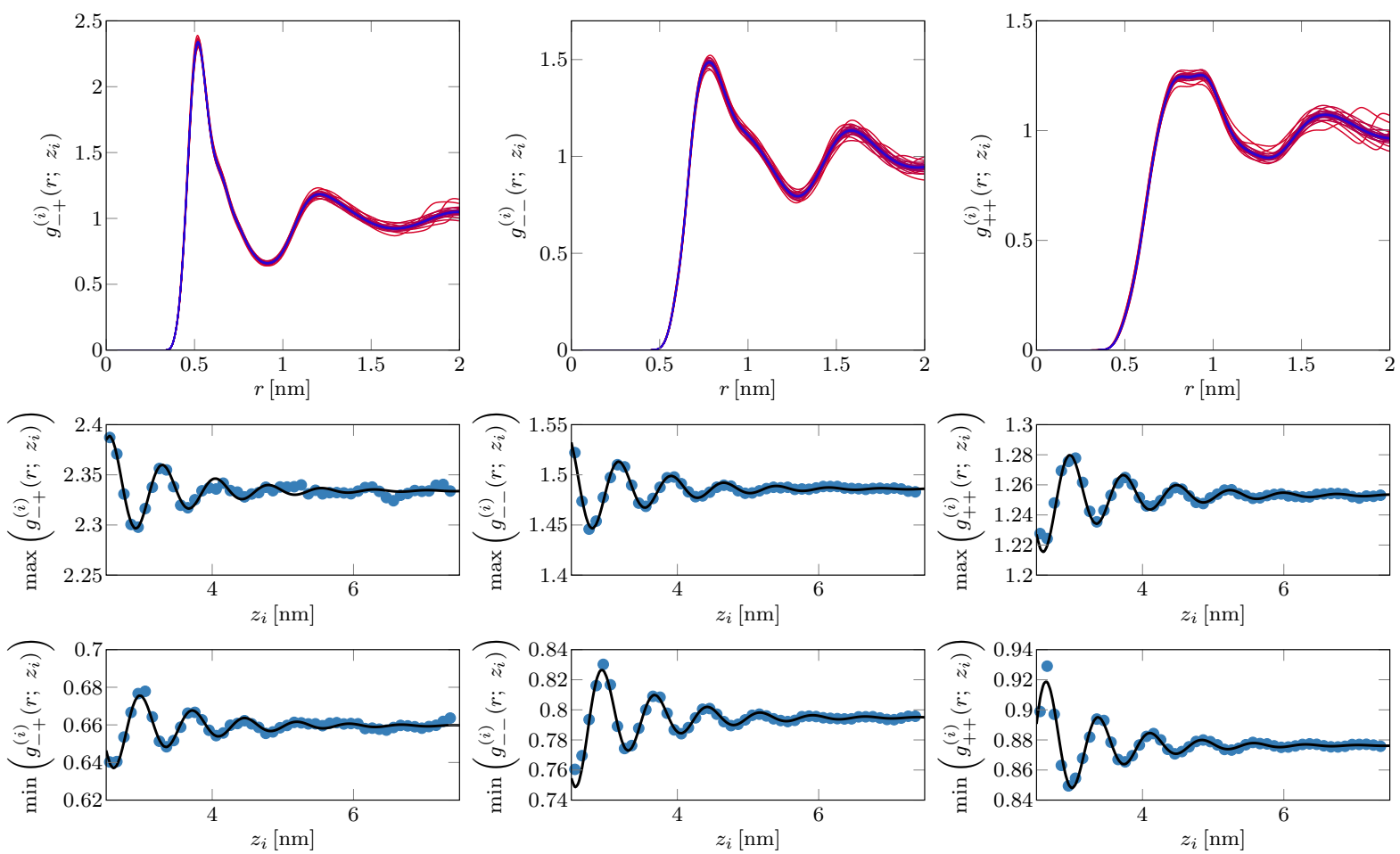

Figure S17: Top row: $z$-distance-dependent $\mathrm{RDFs}_{--+}^{(i)}\left(r ; z_{i}\right)$ (anion-cation, left column), $g_{--}^{(i)}\left(r ; z_{i}\right)$ (anion-anion, middle column), and $g_{++}^{(i)}\left(r ; z_{i}\right)$ (cation-cation, right column) of $\left[\mathrm{C}_{4} \mathrm{C}_{1} \mathrm{Im}\right]^{+}\left[\mathrm{NTf}_{2}\right]^{-}$confined between like-charged graphene walls. The corresponding amplitudes of the first maxima and minima are plotted against the reference distance $z_{i}$ in the middle and bottom row, respectively. The black lines are fits to an oscillatory exponential decay with a decay length varying between 0.9 and $1.1 \mathrm{~nm}$, consistent with the behavior shown for cation-anion RDFs in the main article. 


\section{S2.3 Using reduced-charge ion models in confined systems}

In our simulations of confined ILs, we employed the same reduced-charge model as in our bulk simulations. While reduced charges are used to account for electronic polarization effects in ion-ion interactions (see simulation details in the main article), the same reduced charges do not yield a precise description of solid-liquid interactions. This becomes apparent from the fact that 352 counterions with reduced charge are required to compensate the charge of both walls, whereas a model with full charges would only require about 282 counterions. While the overall charge balance remains correct in our simulations, the volume the additionally required 70 counterions consume would not appear in simulations employing models with full charges. The corresponding change in the total IL volume of about $0.8 \%$ (estimated from 8352 vs. 8282 ions of equal molecular volume) is rather negligible. Nevertheless, as counterions accumulate in higher amounts close to the surfaces, this will likely entail changes in the charge distribution close to the surfaces. While this influence may be non-negligible, according to Kjellander, ${ }^{\mathrm{S} 6}$ the specific interactions of ions with the surface only influence the phase and amplitude of the electric field's long-range decay modes towards the bulk but not their characteristic decay- and wavelengths. We therefore argue that if such decay modes existed in our systems, it should still be possible to observe them at large distances.

We want to stress here that using IL models with full charges will not improve the situation unless electronic polarizability is explicitly taken into account. In fact, non-polarizable models with full charges are known to overestimate interionic Coulomb interactions in ILs and therefore cannot faithfully reproduce interactions with charged surfaces either. Furthermore, as IL models with full charges severely underestimate the mobility of ions, we would expect the confined systems to be practically frozen on time scales accessible to MD simulations if such models were employed.

The formally best approach to describe both ion-surface and bulk ion-ion interactions of ILs more accurately in MD simulations would be to use polarizable force fields, i.e., molecular models with full ionic charges that take electronic polarizability explicitly into account (e.g., by means of inducible point dipoles or Drude oscillators). However, the additional complexity introduced by such models increases the computational cost for simulations of the required length and time scales prohibitively and would have increased the cumulative runtimes of our simulations from months to years. To date, to the best of our knowledge, the approach we chose here yields the most accurate microscopic description of sufficiently large confined IL systems that is at the same time computationally feasible. 


\section{S3 Relating Underscreening to other Measurements}

\section{S3.1 Differential Capacitance Estimates for Different Widths of the Stern Layer}

In the main article, we showed the differential capacitance $C_{\mathrm{d}}$ estimated from from the screening length $\lambda_{\mathrm{S}}$ according to the GCS model

$$
C_{\mathrm{d}}=\left(C_{\text {Stern }}^{-1}+C_{\text {diffuse }}^{-1}\right)^{-1}=\frac{\varepsilon_{0} \varepsilon_{r}}{a+\lambda_{\mathrm{S}}},
$$

where we set $a=d / 2 \approx 0.2 \mathrm{~nm}$. The resulting plot showed a behavior of $C_{\mathrm{d}}$ estimated for screening lengths from SFB measurements (orange dots with dashed line in fig. 9 of the main article) that is qualitatively similar to the original plot by Lee et al. shown in figure 8 of reference S7 but differs in amplitude. In fig. S18 below, we show plots of the very same calculations but with the width of the Stern layer set to $a=2 d \approx 0.8 \mathrm{~nm}$. The results are all qualitatievly similar to those presented in the main article, but the orange line in panel a representing $C_{\mathrm{d}}$ obtained from SFB measurements now reproduces figure 8 of reference S7 exactly. Thus, we conjecture that Lee et al. used $a=$ $2 d$ in their calculations. Note that for this value of $a$, the differential capacity estimate obtained from simulation data (blue line) has a better quantitative and qualitative overall agreement with the direct measurements than all other estimates, and the monotonic behavior of $\lambda_{\mathrm{S}}$ obtained from direct differential capacitance measurements using the inverted GCS model is retained (panel b of fig. S18).

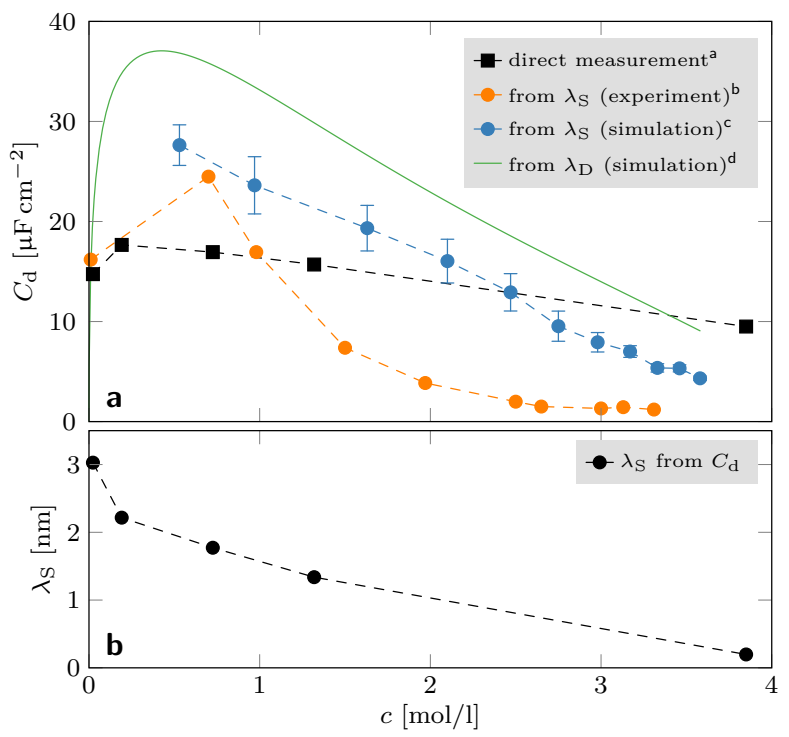

Figure S18: Panel a: Comparison of the concentration-dependent differential capacitance $C_{\mathrm{d}}$ at the point of zero charge obtained from direct measurements ${ }^{\mathrm{S} 8}$ (black squares) and according to the GCS model as defined by eq. (S56) (orange dots: SFB measurements, ${ }^{\mathrm{S} 9}$ blue circles: simulation). The solid green line shows $C_{\mathrm{d}}$ when $\lambda_{\mathrm{S}}$ is replaced by $\lambda_{\mathrm{D}}$ in eq. (S56). Here, we used $a=2 d \approx 0.8 \mathrm{~nm}$ for the evaluation of eq. (S56) instead of $a=d / 2$ in the main article.

${ }^{\text {a }}$ Differential capacitance for $\left[\mathrm{C}_{2} \mathrm{C}_{1} \mathrm{Im}\right]^{+}\left[\mathrm{NTf}_{2}\right]^{-}$from Bozym et al.. ${ }^{\mathrm{S} 8}$

${ }^{\mathrm{b}} \lambda_{\mathrm{S}}$ and $\varepsilon_{r}$ data for $\left[\mathrm{C}_{4} \mathrm{C}_{1} \mathrm{Pyrr}\right]^{+}\left[\mathrm{NTf}_{2}\right]^{-}$from Smith et al.. ${ }^{\mathrm{S} 9}$

${ }^{\mathrm{c}} \lambda_{\mathrm{S}}$ and $\varepsilon_{r}$ data from simulations of $\left[\mathrm{C}_{4} \mathrm{C}_{1} \mathrm{Im}\right]^{+}\left[\mathrm{NTf}_{2}\right]^{-}$(this work).

${ }^{\mathrm{d}} \lambda_{\mathrm{D}}$ was computed using interpolated $\varepsilon_{r}$ data from simulations of $\left[\mathrm{C}_{4} \mathrm{C}_{1} \mathrm{Im}\right]^{+}\left[\mathrm{NTf}_{2}\right]^{-}$(this work).

Panel b: Screening length $\lambda_{\mathrm{S}}$ computed according to the inverted GCS model (eq. (S56) solved for $\lambda_{\mathrm{S}}$ ) with $C_{\mathrm{d}}$ from direct measurements, ${ }^{58} \varepsilon_{r}$ interpolated from simulations, and $a=2 d \approx 0.8 \mathrm{~nm}$.

In both panels, dashed lines serve as a guide to the eye. 


\section{S4 GROMACS Simulation Parameters}

All bulk systems were simulated in the $N p T$ ensemble in cubic boxes with periodic boundary conditions in all three dimensions, where temperature and pressure were maintained at $T=300 \mathrm{~K}$ and $p=1$ bar using a Nosé-Hoover thermostat and Parrinello-Rahman barostat. Short-range van der Waals interactions were computed up to a system-dependent cut-off, and their long-range part was either computed using the smooth particle mesh Ewald (SPME) method with a relative accuracy of $10^{-3}$, or taken into account via analytic dispersion corrections for energy and pressure. Long-range Coulomb interactions were computed using the SPME method with a short-range cut-off of at least $1.3 \mathrm{~nm}$, a relative accuracy of $10^{-5}$ (unless otherwise stated), and tin foil boundary conditions at infinity. The time step of the employed leapfrog integrator was $2 \mathrm{fs}$ for the all-atom models and $5 \mathrm{fs}$ for the coarse-grained IL model.

In the following, we list the GROMACS molecular dynamics parameters used in production runs for all investigated systems.

\section{S4.1 $\left[\mathrm{C}_{4} \mathrm{C}_{1} \mathrm{Im}\right]^{+}\left[\mathrm{PF}_{6}\right]^{-}$(all-atom model)}

\begin{tabular}{|c|c|c|}
\hline & value & Init (note) \\
\hline $\begin{array}{l}\text { integrator } \\
\text { dt } \\
\text { nsteps } \\
\text { comm-mode } \\
\text { nstcomm } \\
\text { nstcalcenergy } \\
\text { nstxout-compressed } \\
\text { compressed-x-precision } \\
\text { cutoff-scheme } \\
\text { nstlist } \\
\text { ns-type } \\
\text { pbc } \\
\text { coulombtype } \\
\text { coulomb-modifier } \\
\text { rcoulomb } \\
\text { vdwtype } \\
\text { vdw-modifier } \\
\text { rvdw } \\
\text { tcoupl } \\
\text { nsttcouple } \\
\text { nh-chain-length } \\
\text { tau-t } \\
\text { ref-t } \\
\text { pcoupl } \\
\text { pcoupltype } \\
\text { nstpcouple } \\
\text { tau-p } \\
\text { compressibility } \\
\text { ref-p } \\
\text { constraints } \\
\text { constraint-algorithm }\end{array}$ & $\begin{array}{l}\text { md } \\
0.002 \\
300000000 \\
\text { linear } \\
50 \\
50 \\
1000 \\
10000 \\
\text { verlet } \\
40 \\
\text { grid } \\
\text { xyz } \\
\text { pme } \\
\text { potential-shift-verlet } \\
1.3 \\
\text { pme } \\
\text { potential-shift-verlet } \\
1.3 \\
\text { nose-hoover } \\
5 \\
1 \\
2.0 \\
300.0 \\
\text { parrinello-rahman } \\
\text { isotropic } \\
5 \\
3.0 \\
4.5 \text { e-5 } \\
1.0 \\
\text { h-bonds } \\
\text { lincs } \\
5\end{array}$ & $\begin{array}{l}\text { (leapfrog integrator) } \\
\text { ps } \\
\text { steps } \\
\text { steps } \\
\text { steps } \\
\text { steps } \\
\text { (means } 10^{-4} \mathrm{~nm} \text { ) } \\
\text { steps }\end{array}$ \\
\hline
\end{tabular}

Table S6: GROMACS molecular dynamics simulation parameters used for the simulation of $\left[\mathrm{C}_{4} \mathrm{C}_{1} \mathrm{Im}\right]^{+}\left[\mathrm{PF}_{6}\right]^{-}$(all-atom model). Only parameters which differ from the default (or have no default) are listed. 


\section{S4.2 $\left[\mathrm{C}_{4} \mathrm{C}_{1} \mathrm{Im}\right]^{+}\left[\mathrm{PF}_{6}\right]^{-}$(coarse-grained model)}

\begin{tabular}{|c|c|c|}
\hline parameter & value & unit (note) \\
\hline $\begin{array}{l}\text { integrator } \\
\text { dt } \\
\text { nsteps } \\
\text { comm-mode } \\
\text { nstcomm } \\
\text { nstcalcenergy } \\
\text { nstxout-compressed } \\
\text { compressed-x-precision } \\
\text { cutoff-scheme } \\
\text { nstlist } \\
\text { ns-type } \\
\text { pbc } \\
\text { coulombtype } \\
\text { coulomb-modifier } \\
\text { rcoulomb } \\
\text { vdwtype } \\
\text { vdw-modifier } \\
\text { dispcorr } \\
\text { rvdw } \\
\text { tcoupl } \\
\text { nh-chain-length } \\
\text { tau-t } \\
\text { ref-t } \\
\text { pcoupl } \\
\text { pcoupltype } \\
\text { tau-p } \\
\text { compressibility } \\
\text { ref-p } \\
\text { constraints } \\
\text { constraint-algorithm }\end{array}$ & $\begin{array}{l}\text { md } \\
0.005 \\
140000000 \\
\text { linear } \\
200 \\
200 \\
1000 \\
1000 \\
\text { verlet } \\
40 \\
\text { grid } \\
\text { xyz } \\
\text { pme } \\
\text { potential-shift-verlet } \\
1.6 \\
\text { cut-off } \\
\text { potential-shift-verlet } \\
\text { enerpres } \\
1.6 \\
\text { nose-hoover } \\
1 \\
5.0 \\
300.0 \\
\text { parrinello-rahman } \\
\text { isotropic } \\
10.0 \\
4.5 \mathrm{e}-5 \\
1.0 \\
\text { all-bonds } \\
\text { lincs }\end{array}$ & $\begin{array}{l}\text { (leapfrog integrator) } \\
\text { ps } \\
\text { steps } \\
\text { steps } \\
\text { steps } \\
\text { steps } \\
\text { (means } 10^{-3} \mathrm{~nm} \text { ) } \\
\text { steps }\end{array}$ \\
\hline
\end{tabular}

Table S7: GROMACS molecular dynamics simulation parameters used for the simulation of $\left[\mathrm{C}_{4} \mathrm{C}_{1} \mathrm{Im}\right]^{+}\left[\mathrm{PF}_{6}\right]^{-}$(coarse-grained model). Only parameters which differ from the default (or have no default) are listed. 


\section{S4.3 Aqueous $\mathrm{NaCl}$ solutions}

\begin{tabular}{|c|c|c|}
\hline parameter & value & unit (note) \\
\hline $\begin{array}{l}\text { integrator } \\
\text { dt } \\
\text { nsteps } \\
\text { comm-mode } \\
\text { nstcomm } \\
\text { nstcalcenergy } \\
\text { nstxout-compressed } \\
\text { compressed-x-precision } \\
\text { cutoff-scheme } \\
\text { nstlist } \\
\text { ns-type } \\
\text { pbc } \\
\text { coulombtype } \\
\text { coulomb-modifier } \\
\text { rcoulomb } \\
\text { vdwtype } \\
\text { vdw-modifier } \\
\text { dispcorr } \\
\text { rvdw } \\
\text { ewald-rtol } \\
\text { tcoupl } \\
\text { nsttcouple } \\
\text { nh-chain-length } \\
\text { tau-t } \\
\text { ref-t } \\
\text { pcoupl } \\
\text { pcoupltype } \\
\text { nstpcouple } \\
\text { tau-p } \\
\text { compressibility } \\
\text { ref-p } \\
\text { constraints } \\
\text { constraint-algorithm }\end{array}$ & $\begin{array}{l}\text { md } \\
0.002 \\
100000000 \\
\text { linear } \\
50 \\
50 \\
1000 \\
10000 \\
\text { verlet } \\
40 \\
\text { grid } \\
\text { xyz } \\
\text { p3m-ad } \\
\text { potential-shift-verlet } \\
1.2 \\
\text { cut-off } \\
\text { potential-shift-verlet } \\
\text { enerpres } \\
1.2 \\
1.0 \mathrm{e}-6 \\
\text { nose-hoover } \\
5 \\
1 \\
2.0 \\
300.0 \\
\text { parrinello-rahman } \\
\text { isotropic } \\
5 \\
3.0 \\
4.5 \mathrm{e}-5 \\
1.0 \\
\text { all-bonds } \\
\text { lincs } \\
5\end{array}$ & $\begin{array}{l}\text { (leapfrog integrator) } \\
\text { ps } \\
\text { steps } \\
\text { steps } \\
\text { steps } \\
\text { steps } \\
\left.\text { (means } 10^{-4} \mathrm{~nm}\right) \\
\text { steps }\end{array}$ \\
\hline
\end{tabular}

Table S8: GROMACS molecular dynamics simulation parameters used for the simulation of aqueous $\mathrm{NaCl}$ solutions. Only parameters which differ from the default (or have no default) are listed. 


\section{S4.4 $\left[\mathrm{C}_{4} \mathrm{C}_{1} \mathrm{Im}\right]^{+}\left[\mathrm{NTf}_{2}\right]^{-}$in PC}

\begin{tabular}{|c|c|c|}
\hline parameter & value & unit (note) \\
\hline $\begin{array}{l}\text { integrator } \\
\text { dt } \\
\text { nsteps } \\
\text { comm-mode } \\
\text { nstcomm } \\
\text { nstcalcenergy } \\
\text { nstxout-compressed } \\
\text { compressed-x-precision } \\
\text { cutoff-scheme } \\
\text { nstlist } \\
\text { ns-type } \\
\text { pbc } \\
\text { coulombtype } \\
\text { coulomb-modifier } \\
\text { rcoulomb } \\
\text { vdwtype } \\
\text { vdw-modifier } \\
\text { dispcorr } \\
\text { rvdw } \\
\text { tcoupl } \\
\text { nsttcouple } \\
\text { nh-chain-length } \\
\text { tau-t } \\
\text { ref-t } \\
\text { pcoupl } \\
\text { pcoupltype } \\
\text { nstpcouple } \\
\text { tau-p } \\
\text { compressibility } \\
\text { ref-p } \\
\text { constraints } \\
\text { constraint-algorithm }\end{array}$ & 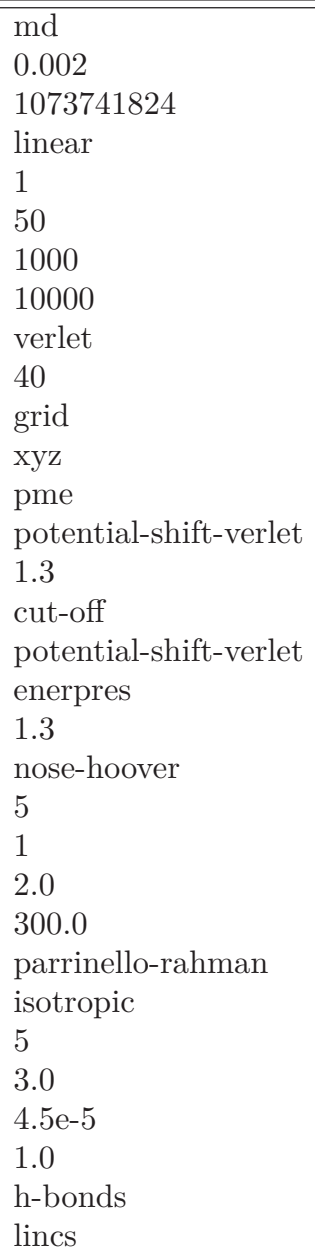 & $\begin{array}{l}\text { (leapfrog integrator) } \\
\text { ps } \\
\text { steps }\left(=2^{30}\right) \\
\text { steps } \\
\text { steps } \\
\text { steps } \\
\left.\text { (means } 10^{-4} \mathrm{~nm}\right) \\
\text { steps }\end{array}$ \\
\hline
\end{tabular}

Table S9: GROMACS molecular dynamics simulation parameters used for the simulation of $\left.\left[\mathrm{C}_{4} \mathrm{C}_{1} \mathrm{Im}\right]^{+}[\mathrm{NTf}]_{2}\right]^{-}$in propylene carbonate. Only parameters which differ from the default (or have no default) are listed. 


\section{S4.5 $\left[\mathrm{C}_{4} \mathrm{C}_{1} \mathrm{Im}\right]^{+}\left[\mathrm{NTf}_{2}\right]^{-}$confined between like-charged graphene walls}

\begin{tabular}{|c|c|c|}
\hline param & value & \\
\hline $\begin{array}{l}\text { integrator } \\
\text { dt } \\
\text { nsteps } \\
\text { comm-mode } \\
\text { nstcomm } \\
\text { nstcalcenergy } \\
\text { nstxout-compressed } \\
\text { compressed-x-precision } \\
\text { cutoff-scheme } \\
\text { nstlist } \\
\text { ns-type } \\
\text { pbc } \\
\text { coulombtype } \\
\text { coulomb-modifier } \\
\text { rcoulomb } \\
\text { vdwtype } \\
\text { vdw-modifier } \\
\text { dispcorr } \\
\text { rvdw } \\
\text { ewald-geometry } \\
\text { tcoupl } \\
\text { nsttcouple } \\
\text { tau-t } \\
\text { ref-t } \\
\text { pcoupl } \\
\text { pcoupltype } \\
\text { nstpcouple } \\
\text { tau-p } \\
\text { compressibility } \\
\text { ref-p } \\
\text { constraints } \\
\text { constraint-algorithm } \\
\text { nwall } \\
\text { wall-type } \\
\text { wall-r-linpot } \\
\text { wall-atomtype } \\
\text { wall-density } \\
\text { wall-ewald-zfac }\end{array}$ & $\begin{array}{l}\text { md } \\
0.002 \\
500000000 \\
\text { linear } \\
50 \\
50 \\
1000 \\
10000 \\
\text { verlet } \\
40 \\
\text { grid } \\
\text { xy } \\
\text { pme } \\
\text { potential-shift-verlet } \\
1.3 \\
\text { pme } \\
\text { potential-shift-verlet } \\
\text { no } \\
1.3 \\
3 \text { dc } \\
\text { v-rescale } \\
-1 \\
2.0 \\
300.0 \\
\text { no } \\
\text { semiisotropic } \\
-1 \\
3.0 \\
0.0 \quad 4.5 \text { - } 5 \\
1.0 \quad 1.0 \\
\text { h-bonds } \\
\text { lincs } \\
2 \\
\text { harmonic } \\
-1 \\
\text { WALL WALL } \\
1.0 \quad 1.0 \\
3 \\
\end{array}$ & $\begin{array}{l}\text { steps } \\
\text { ps } \\
\mathrm{K} \\
\text { (for equilibration: parrinello-rahman) } \\
\text { steps } \\
\text { ps } \\
\text { bar }^{-1} \\
\text { bar } \\
\text { (fix length of bonds involving } \mathrm{H} \text { atoms) }\end{array}$ \\
\hline
\end{tabular}

Table S10: GROMACS molecular dynamics simulation parameters used for the simulation of $\left.\left[\mathrm{C}_{4} \mathrm{C}_{1} \mathrm{Im}\right]^{+}[\mathrm{NTf}]_{2}\right]^{-}$confined between likecharged graphene walls. Only parameters which differ from the default (or have no default) are listed. The wall type "harmonic" is a custom extension to GROMACS, which is used to couple each graphene wall to its corresponding simulation box face with a harmonic potential. This was necessary during $N p T$ equilibration because the current implementation of reference coordinate scaling does not work in conjunction with semiisotropic pressure coupling. During production runs $(N V T$ ensemble, no pressure coupling), using this wall type has exactly the same effect as applying harmonic position restraints (force constant $10000 \mathrm{~kJ} \mathrm{~mol}^{-1}$ ) to the wall carbon atoms in the $z$-direction. To constrain the atomic positions in the $x$ - and $y$-directions, normal position restraints with the same force constant are used. Note that for correct pressure estimates, the forces due to position restraints of the wall atoms and also the forces between atoms within each wall have to be excluded from the virial computation. Otherwise, intra-wall forces would invalidate the pressure computation. 


\section{References}

[S1] Neumann, M. Dipole moment fluctuation formulas in computer simulations of polar systems. Molecular Physics 1983, 50, 841-858, DOI: 10.1080/00268978300102721.

[S2] Sega, M.; Kantorovich, S. S.; Arnold, A.; Holm, C. In Recent Advances in Broadband Dielectric Spectroscopy; Kalmykov, Y. P., Ed.; NATO Science for Peace and Security Series B: Physics and Biophysics; Springer Netherlands, 2013; pp 103-122, DOI: 10.1007/978-94-007-5012-8_8.

[S3] Machida, H.; Sato, Y.; Smith Jr., R. L. Pressure-volume-temperature (PVT) measurements of ionic liquids $\left(\left[\mathrm{bmim}^{+}\right]\left[\mathrm{PF}_{6}^{-}\right],\left[\mathrm{bmim}^{+}\right][\mathrm{BF} 4-],\left[\mathrm{bmim}^{+}\right]\left[\mathrm{OcSO}_{4}^{-}\right]\right)$and analysis with the SanchezLacombe equation of state. Fluid Phase Equilibria 2008, 264, 147-155, DOI: 10.1016/j.fluid. 2007.11 .005 .

[S4] Vraneš, M.; Zec, N.; Tot, A.; Papović, S.; Dožić, S.; Gadžurić, S. Density, electrical conductivity, viscosity and excess properties of 1-butyl-3-methylimidazolium bis(trifluoromethylsulfonyl)imide + propylene carbonate binary mixtures. The Journal of Chemical Thermodynamics 2014, 68, 98-108, DOI: $10.1016 / j \cdot j c t .2013 .08 .034$.

[S5] Rumble, J. R., Ed. CRC Handbook of Chemistry and Physics, 100th ed.; CRC Handbook of Chemistry and Physics; CRC Press / Taylor \& Francis: Boca Raton, Florida, 2019.

[S6] Kjellander, R. Focus Article: Oscillatory and long-range monotonic exponential decays of electrostatic interactions in ionic liquids and other electrolytes: The significance of dielectric permittivity and renormalized charges. The Journal of Chemical Physics 2018, 148, 193701, DOI: $10.1063 / 1.5010024$.

[S7] Lee, A. A.; Perez-Martinez, C. S.; Smith, A. M.; Perkin, S. Underscreening in concentrated electrolytes. Faraday Discussions 2017, 199, 239-259, DOI: 10.1039/c6fd00250a.

[S8] Bozym, D. J.; Uralcan, B.; Limmer, D. T.; Pope, M. A.; Szamreta, N. J.; Debenedetti, P. G.; Aksay, I. A. Anomalous Capacitance Maximum of the Glassy Carbon-Ionic Liquid Interface through Dilution with Organic Solvents. The Journal of Physical Chemistry Letters 2015, 6, 2644-2648, DOI: 10.1021/acs.jpclett.5b00899.

[S9] Smith, A. M.; Lee, A. A.; Perkin, S. The Electrostatic Screening Length in Concentrated Electrolytes Increases with Concentration. The Journal of Physical Chemistry Letters 2016, 7, 2157-2163, DOI: 10.1021/acs.jpclett.6b00867. 